•技术与方法・

\title{
国内8 款常用植物识别软件的识别能力评价
}

\author{
许展慧 ${ }^{1}$ 刘诗尧 ${ }^{1}$ 赵 莹 ${ }^{1}$ 涂文琴 ${ }^{1}$ 常诏峰 ${ }^{1}$ 张恩涛 ${ }^{1}$ 郭 靖 ${ }^{1}$ \\ 郑 迪 ${ }^{1}$ 耿 鏊 ${ }^{1}$ 顾高营 ${ }^{1}$ 郭淳鹏 $^{1}$ 郭璐璐 ${ }^{1}$ 王 静 ${ }^{1}$ 徐春阳 $^{1}$ \\ 彭 钏 ${ }^{1}$ 杨 腾 $^{1}$ 崔梦琪 $^{1}$ 孙伟成 $^{1}$ 张剑坛 $^{1}$ 刘皓天 $^{1}$ 巴超群 ${ }^{1}$ \\ 王鹤琪 $^{1}$ 贾竟超 ${ }^{1}$ 武金洲 ${ }^{1}$ 肖 翠 $^{2}$ 马克平 $2^{*}$ \\ 1 (中国科学院大学, 北京 100049) \\ 2 (中国科学院植物研究所植被与环境变化国家重点实验室, 北京 100093)
}

\begin{abstract}
摘要: 随着智能手机和人工智能技术的发展, 以手机app为载体的植物识别软件慢慢走进公众生活、科普活动和科 研活动的各个方面。植物识别app的识别正确率是决定其使用价值和用户体验的关键因素。目前, 国内应用市场上 有许多植物识别app, 它们的开发目的和应用范围各异, 软件本身的关注点、数据库来源、算法、硬件要求也存在 很大差异。对于不同人群, 植物识别app有不同的意义, 如对于科研人员来说, 识别能力强的app是提高效率的一大 工具; 对植物爱好者来说, 具一定准确率的识别app可以作为入门的工具。因此, 对各app的识别能力进行分析与评 价显得尤为重要。本文选取了 8 款常用的app, 分别对400张已准确鉴定的植物图片进行识别, 其中干旱半干旱区、 温带、热带和亚热带4个区各选取100张。这些图片共计122科164属340种, 涵盖了乔木、灌木、草本、草质藤本和 木质藤本 5 种生长型, 包含 23 种国家级保护植物。种、属、科准确识别正确分别计 4 分、2分、1分, 以此标准对软 件识别能力按总得分进行排序, 正确率得分由高到低依次为花帮主、百度识图、花伴侣、形色、花卉识别、植物 识别、发现识花、微软识花。
\end{abstract}

关键词: 植物识别软件; 花帮主; 百度识图; 花伴侣; 形色; 花卉识别; 植物识别; 发现识花; 微软识花

\section{Evaluation of the identification ability of eight commonly used plant identification application softwares in China}

Zhanhui Xu ${ }^{1}$, Shiyao Liu ${ }^{1}$, Ying Zhao ${ }^{1}$, Wenqin $\mathrm{Tu}^{1}$, Zhaofeng Chang ${ }^{1}$, Entao Zhang ${ }^{1}$, Jing Guo ${ }^{1}$, Di Zheng ${ }^{1}$, Jun Geng ${ }^{1}$, Gaoying $\mathrm{Gu}^{1}$, Chunpeng Guo ${ }^{1}$, Lulu Guo ${ }^{1}$, Jing Wang ${ }^{1}$, Chunyang $\mathrm{Xu}^{1}$, Chuan Peng ${ }^{1}$, Teng Yang ${ }^{1}$, Mengqi Cui ${ }^{1}$, Weicheng Sun ${ }^{1}$, Jiantan Zhang ${ }^{1}$, Haotian Liu ${ }^{1}$, Chaoqun Ba ${ }^{1}$, Heqi Wang ${ }^{1}$, Jingchao Jia ${ }^{1}$, Jinzhou $\mathrm{Wu}^{1}$, Cui Xiao ${ }^{2}$, Keping $\mathrm{Ma}^{2 *}$

1 University of Chinese Academy of Sciences, Beijing 100049

2 State Key Laboratory of Vegetation and Environmental Change, Institute of Botany, Chinese Academy of Sciences, Beijing 100093

\begin{abstract}
Smart phone and artificial intelligence technology development has led to various plant recognition softwares on mobile applications. These applications have gradually entered all aspects of public life, popular science activities, and scientific research activities. Presently, there are many plant recognition apps in China, which have varying development purposes and application scopes. Among these differences include variation in software concerns, database sources, algorithms, and hardware which could implicate large discrepancies between apps, making it important to analyze and evaluate the accuracy, scope of application and potential use of each software. In this paper, eight apps were selected to identify 400 accurately identified plant photos, 100 photos being chosen from arid and semi-arid zones, temperate zones, tropical zones, and subtropical zones, respectively. In total, these photos belong to 122 families, 164 genera and 340 species, covering five growth forms of trees, shrubs, herbs, herbaceous vines and woody vines, as
\end{abstract}

收稿日期: 2019-09-01; 接受日期: 2020-01-21

基金项目: 中国科学院战略性先导科技专项(XDA19050404)

* 通讯作者 Author for correspondence. E-mail: kpma@ibcas.ac.cn 
well as 23 national protected plant species. Accurate identification of species, genera and families was scored 4, 2 and 1 points, respectively. The software recognition ability was sorted according to total scores, and the results are as follows: HuaBangZhu, Baidu-Shitu, HuaBanLv, XingSe, Huahui-Shibie, Zhiwu-Shibie, Faxian-Shihua, Flower Recognition.

Key words: Plant recognition software; HuaBangZhu; Baidu-Shitu; HuaBanLv; XingSe; Huahui-Shibie; Zhiwu-Shibie; Faxian-Shihua; Flower recognition

植物分类学是一门传统基础学科, 对植物进行 分类识别对生物多样性保护、生态学、林学、园林、 生物安全等领域均有重要意义。传统的植物识别与 分类主要由人工完成, 对专业水平要求高、工作量 大、耗时长, 且难以保证客观性(曹香漟等, 2018)。 随着科学研究和科普事业的发展, 科研人员及大众 对准确便捷识别植物的需求不断增加。

利用计算机技术进行植物种类识别是最近 30 年的事情。国外的研究机构已经有过一些相关的分 析和研究, 国内利用计算机技术进行植物种类识别 起步比较晚, 在 20 世纪90年代中期才开始进行相关 的研究, 并开始尝试将这方面的技术应用在农业生 产领域 ${ }^{(1)}$ 。

在开发植物识别软件的初期, 识别对象主要是 植物叶片, 多为基于关系结构匹配、统计学和机器 学习的识别方法(张宁和刘文萍, 2011)。植物叶片具 有的扁平叶面结构在拍照处理、特征提取和技术建 模上具有优势, 但缺点是叶片的性状不稳定, 易受 环境影响, 且不同物种因为适应环境可能会出现相 似的性状 ${ }^{(1)}$ 。相比之下, 花是植物更为稳定的性状, 因此以花朵为依据进行植物的分类识别更加可靠, 也是植物识别app的发展趋势。随着深度学习(deep learning)理论的发展, 卷积神经网络(convolutional neural networks)逐渐推广应用, 早期出现的过拟合 问题在一定程度上得到解决。随着机器学习方法应 用更加广泛, 植物识别器官也扩展到花、果实和种 子等(张宁和刘文萍, 2011)。

早期的植物识别软件主要安装在PC端, 有不 便携带、费用高、难以普及等局限。在手机端植物 识别软件出现之前, 有些高校开发出了一些局部地 区植物识别软件, 如浙江农林大学“校园植物通”结 合校园地图记录了校园内2,500多种植物, 可先识 别植物的位置, 再分析植物的特征, 从数据库中提

(1) 张岩 (2017) 植物花朵识别系统的设计与实现. 硕士学位论文, 华中 科技大学, 武汉.
取植物的信息(张健捷等, 2017)。但这只能满足查 询、记录的功能, 利用定位信息从数据库中提取结 果, 严格来说算不上真正意义的识别。北京林业大 学的学生团队利用误差反向传播(back propagation, BP)神经网络技术开发了植物叶片识别系统一百 叶通客户端, 取得了较好的效果, 但其识别的植物 种类依然很有限(钟刚亮等, 2015)。张玉玩和李柏依 (2016)设计了云计算与移动终端结合的植物远程快 速识别系统, 即通过上传植物图像至云服务器, 将 其与叶片标准库中的图像进行比对以识别植物, 取 得了一定的成果。王礼等(2018)基于iOS操作系统设 计了一款利用叶片识别观赏植物的应用程序, 并选 取了浙江农林大学校园内的 8 种常见观赏植物进行 测评, 发现平均识别率为 $92 \%$, 平均用时 $2.6 \mathrm{~s}$ 。

在技术成熟之后，国外开发了一批相对优秀但 关注点各不相同的手机应用软件。Pl@ntNet是2009 年以来由Agropolis Foundation支持的植物生物多样 性研究和教育计划。该计划开发的植物识别功能完 全免费公开，同时支持Web端和手机端(https:// identify.plantnet.org/)。其中手机端app名为PlantNet Plant Identification, 可识别大约20,000种有花植物 和蕨类植物(http://play.google.com/store/apps/details? $\mathrm{id}=$ org.plantnet\&hl/)。Leafsnap是第一个使用自动视 觉识别植物的移动应用程序, 可离线使用。它由哥 伦比亚大学、马里兰大学和史密森学会于2011年合 作开发, 该软件可利用花朵、果实、叶柄、种子和 树皮的照片来识别树木(http://leafsnap.com/about/)。 该系统用计算机视觉组件分割叶的形态、提取多尺 度上代表叶轮廓曲率的特征, 以此对植物加以识别 (Kumar et al, 2012)。Leafsnap分英国版本和美国版 本, 能识别英国的156种树种, 美国东北部的185种 树种, 关于英国、美国的物种信息较全面, 包括生 长型、栖息地类型、开花时间和分布等 (http:// leafsnap.com/dataset/; https://www.nhm.ac.uk/take-part/ identify-nature/leafsnap-uk-app.html)。PlantSnap软件 
于2012年面世，识别类型涵盖花卉、树木、蘑菇、 多肉植物, 目前该软件数据库中有 625,000 多种, 并 将持续把更多物种添加到训练算法中 (https:// www.plantsnap.com/)。2015年, LikeThat Garden发布, 除植物外, 该app还可识别蝴蝶。识别完成后, 可跳 转维基百科, 提供更详尽的物种信息, 同时还有带 定位分享结果的功能 (https://appadvice.com/app/ likethat-garden-flower-identification/)。

在我国, 植物识别app的发展离不开互联网创 业公司“互联网+”创新思维, 与国家在中国科学院 “一三五”规划中提出的建设“90个国家花卉种质资 源库”、中国科学院植物研究所部署的“数字植物与 物种快速鉴定”等项目也密切相关 ${ }^{(1)}$ 。2010年12月13 日, 百度识图测试版上线, 这是百度公司推出的一 项搜索相似图片的服务。用户可以上传本地图片或 输入图片的url地址来进行搜索, 随后百度公司把百 度识图与百度图片搜索集成。百度识图不仅具有相 同/相似图像搜索、全网人脸搜索等功能, 也能够辨 别植物。对于识别的植物, 直接跳转百度百科, 可 获得更详尽的描述。这也是最初使用百度网站识别 植物的方法。截至目前, 百度识图只是百度App上 的一个子功能, 没有独立的手机移动端应用, 但其 仍具有相当大的潜力。2015年8月, 形色上线。形色 App由杭州睿琪软件有限公司与中国自然标本馆 (CFH) 合作开发, 其他合作单位还有中国科学院植 物研究所和中国科学院上海辰山植物园等(李珉葶, 2017)。除了鉴别花卉, 形色还为用户搭建了一个功 能更丰富的社交平台。形色App的识别结果包括植 物种属、花语、植物价值、植物养护等，同时还有 景点推荐、热点话题和鉴定求助等功能(王加希, 2019)。2016年9月, 微软识花上线, 其关注点主要集 中在花卉识别。它由微软亚洲研究院和中国科学院 植物研究所携手研发, 应用深度学习技术, 基于中 国科学院植物研究所的 260 万张花的鉴定照片对机 器识别模型进行训练。特别的是, 它是一款离线应 用, 可在无法连接互联网的场所使用。2016年10月, 鲁朗软件有限公司与中国科学院植物研究所合作, 利用数百万张植物彩色照片开发的植物识别app花 伴侣上线, 这是一款基于大数据和深度学习算法的 产品, 拥有数量庞大的学习训练图片和可靠的分类

(1) 林心怡 (2017) 我国植物辨识科普发展研究. 硕士学位论文, 西北农 林科技大学, 杨凌.
学信息(王桢和何建勇, 2017), 以中国植物图像库海 量植物分类图片为基础，可识别 5,000种常见植物 (李敏, 2018)。花帮主是一款定位为“养花者的大本 营”的软件，与花伴侣为同一团队所运行，包含养 花问题解答、园艺知识和相关资讯播报等功能，亦 有着较高的识别率，并且对温带地区的野外植物具 有识别优势。

随着智能手机的快速普及, 基于深度学习的新 一代植物识别算法不断完善, 促成多个植物识别 app面世。深度学习技术在植物识别中的应用过程 中，训练算法所用的图片质量与算法本身的优劣同 样重要, 而高像素、大内存的智能手机满足了这一 要求。总体而言, 促成当前植物识别软件快速发展 的条件有 4 个: (1)海量且准确鉴定的植物图片; (2)先 进的人工智能算法; (3) 良好的存储和运算能力以及 网络传输能力; (4)高性能智能手机的快速普及。智 能手机及其应用程序可以替代许多传统的手持式 传感器、计算器和数据存储设备。

但各个app的服务定位及功能各有侧重，识别 能力也有所差异。本文选取了8款国内目前较热门的 植物识别app, 希望对它们的识别能力进行基本的 分析与评价。

\section{数据来源与方法}

\section{1 数据来源}

将中国植被区划的 8 个区域中植物区系相对相 似的区域归并, 选择温带区(包括寒温带针叶林区、 温带针阔混交林区和暖温带落叶阔叶林区)、亚热带 区(亚热带常绿阔叶林区)、热带区(热带雨林、季雨 林区)和干旱半干旱区(温带草原区和温带荒漠区) 4 个区。然后，在中国自然标本馆网站(http://www.cfh. ac.cn/)分别从每个区选择 100 张图片作为基本数据 集，用以比较不同软件的植物识别能力。为了便于 更多人可进行重复测试，已将鉴定准确的 400 张照 片上传至NSII服务器(http://www.nsii.org.cn/2017/ testPhoto.php/)。这些照片共计有植物122科164属 340 种, 以野生种为主, 少量栽培植物。在 400 张照 片中, 有乔木 98 张，灌木 99 张，草本 180 张，木质藤 本13张，草质藤本 10 张。根据国家重点保护野生植 物名录, 这 400 张照片中包含国家一、二级保护植物 图片23张。 


\section{2 研究方法}

测试时间为2019年3月。识别测试的基本单元 为照片, 无空白对照, 无重复, 仅记录每张图片首 次识别的结果。移动端识别分为本地识别和远程识 别, 在此采用本地识别的方法。首先选出测试所用 的软件: 从国内的应用商店优化(App Store Optimization)网站以“植物识别”为搜索关键词, 比较了七 麦数据(https://www.qimai.cn/)、蝉大师(https://www. chandashi.com/) 以及德普优化(https://www.deepaso. $\mathrm{com} /$ ) 3 个应用商店优化网站的结果后, 选取了与关 键词相关性较强、下载量较大、评分相对较高的7 款软件, 加上具有百度识图功能的百度App, 总计 8 款软件, 即花伴侣、形色、花帮主、百度识图、微 软识花、植物识别、花卉识别、发现识花。第二步, 整理待识别植物的物种名及科名和属名的中文名 与拉丁名。第三步, 将 4 个区的共 400 张图片存入手 机相册。最后, 用选取的 8 款软件分别对图片进行识 别, 控制测试环境(网络环境、手机内存足够)的统一, 记录识别结果的中文名、拉丁名、科名和属名。每 个识别软件都会给出中文种名, 但由于植物的中文 种名很多存在同物异名现象, 因此对不一致的中文 种名需要核实其拉丁名。对于识别结果中没有拉丁 名的植物, 以中国植物志网站(http://frps.iplant.cn/) 上检索出的拉丁名为准。

大部分识别软件的识别结果并不具唯一性, 会 有多个备选结果, 对于这样的情况, 选择其中可能 性最高的作为识别的结果。将结果整理成表格, 按 照计分标准记录各软件对每种植物的识别得分。

\section{3 分析方法}

以照片为测试的基本单元, 按照生物分类阶元 识别的难易程度赋予不同的分值: 识别结果精确到 种, 计 4 分, 精确到属, 计 2 分, 精确到科, 计 1 分, 识 别错误则计 0 分。种是最重要的分类阶元, 还可与现 实存在的物种一一对应, 故正确识别到种得分最高

在获得 8 款软件对每一张照片的识别结果后, 记录具体分值, 将每个软件的具体分值相加, 进行 如下排序: 总得分排序、不同气候研究区的识别结 果得分排序、对不同生长型的识别结果排序。对于 各软件针对不同生长型以及国家一、二级重点保护 植物识别到科、属、种的结果用比例来表示(因为样 本中5种生长型的数量不同), 再进行比较和排序。

\section{结果}

\section{1 总得分排序}

从总体的识别结果来看, 花帮主得分最高, 其 次是百度识图、花伴侣、形色, 这4款app的得分较 高且比较接近, 与其余 4 款相比有较明显的差异(图 1)。在排名较后的 4 款app中, 花卉识别与植物识别 的得分较接近, 而与发现识花和微软识花有比较明 显的差异。

\section{2 对不同气候区图片的识别结果}

对于干旱半干旱区、温带、亚热带、热带这4 个不同的气候区, 除了亚热带区外, 均是花帮主的 得分最高(图2)。而百度识图在亚热带区表现最佳, 且在其他地区得分也较高。8个app在干旱半干旱区 的得分均低于其他 3 个区。在识别准确率最高的 4 款

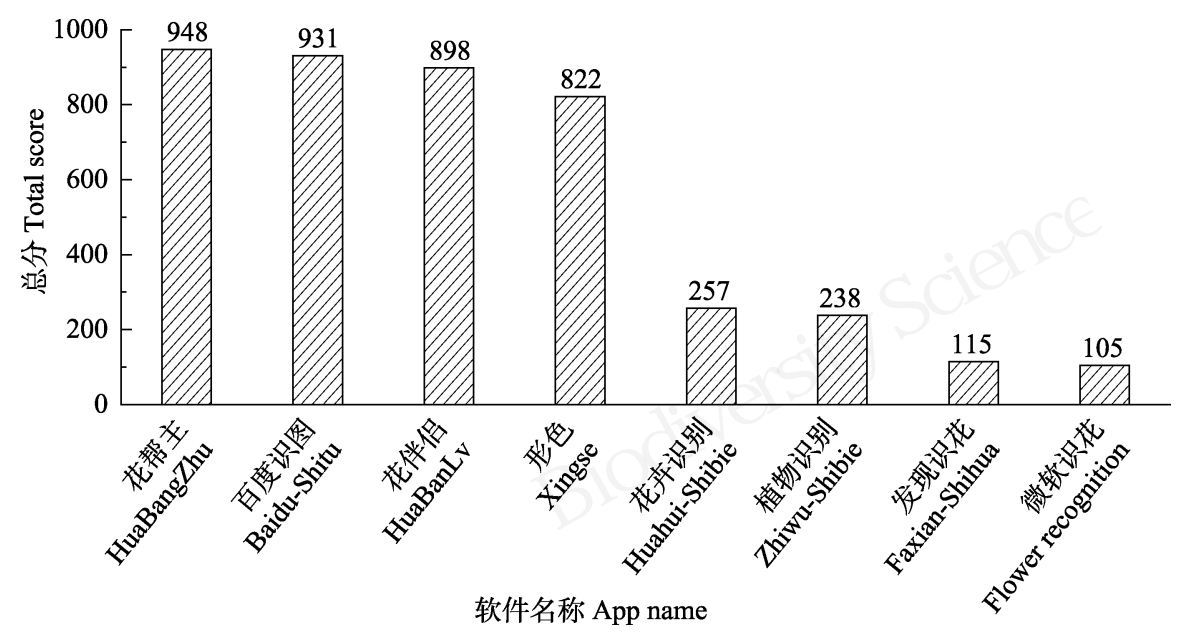

图1 国内8款常用植物识别软件总得分排序

Fig. 1 Total score ranking of eight commonly used plant identification application softwares in China 
软件中, 花帮主表现最佳, 但在亚热带区, 其识别 准确率却排在百度识图、花伴侣、形色之后。百度 识图在 4 个区的表现都较稳定。花伴侣与形色也在 4 个区都有较高的识别率，且在亚热带区表现较好， 在其他区的表现次于花帮主和百度识图。

就单个软件来说，花帮主在 4 个区的得分都较 高, 其中识别效果最佳的是在温带区; 百度识图在 4 个区的得分也较高, 且更为均衡, 其中识别效果 最佳的是在亚热带区; 花伴侣的得分仅次于花帮主 和百度识图, 在温带区、亚热带区表现均较好; 形 色的整体得分较高，在热带和亚热带区表现最好; 微软识花、植物识别、花卉识别、发现识花这 4 款 app表现最好的区分别是: 温带以及干旱半干旱区、
温带区、热带区(图2)。

\section{3 对于不同生长型的识别结果}

对不同生长型的识别结果进行比较发现，花伴 侣、形色、花帮主、百度识图对乔木、灌木、草本、 木质藤本、草质藤本 5 种生长型的识别得分更高，且 4 款app间的分差别不大，总体而言以花帮主略占优 势，形色稍逊于其他 3 款。微软识花、植物识别、花 卉识别、发现识花虽得分不如上述4款app, 但也有 一定的识别效果，其中，植物识别、花卉识别的识别 效果要好于微软识花和发现识花(图3)。

对于乔木的识别，花伴侣、形色、花帮主、百 度识图4款app正确识别(即识别到种)的比例在 $50 \%$ 左右，以花帮主的53\%为最高。微软识花、植物识

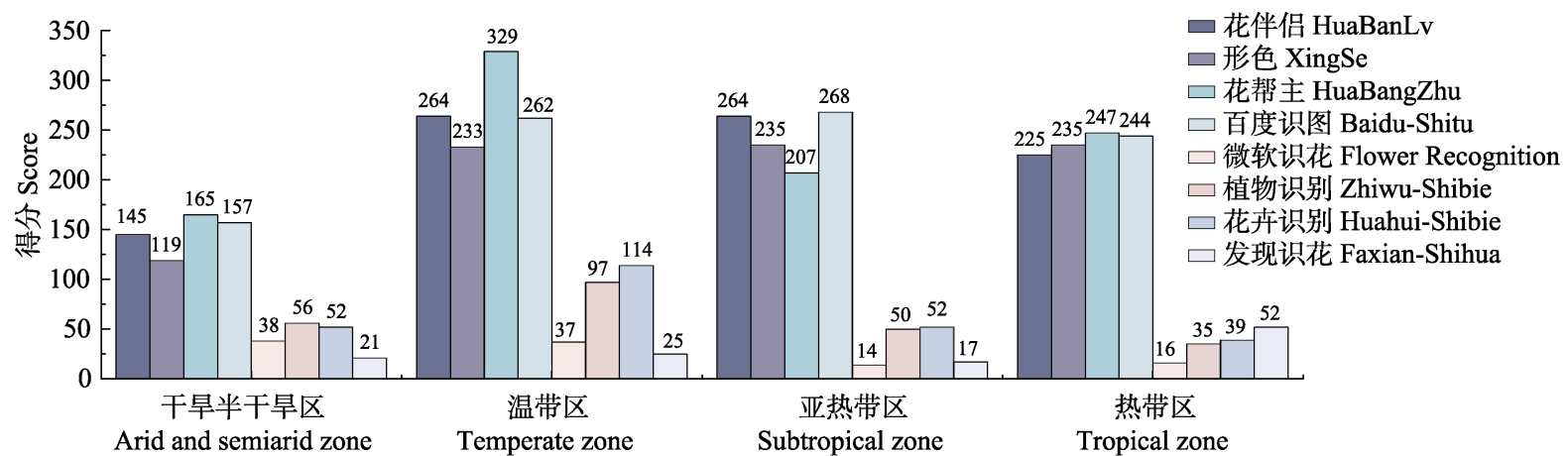

气候区 Climate zone

图2 国内 8 款常用植物识别软件在气候区的得分排序

Fig. 2 Score ranking by climate zone of eight commonly used plant identification application softwares in China

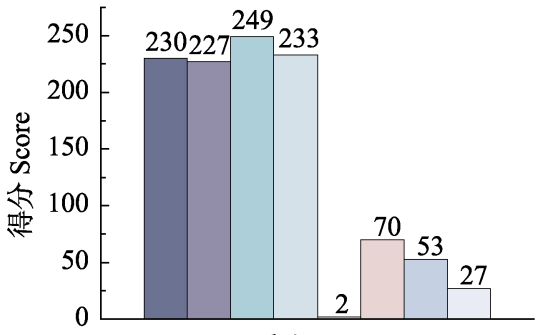

乔木 Tree

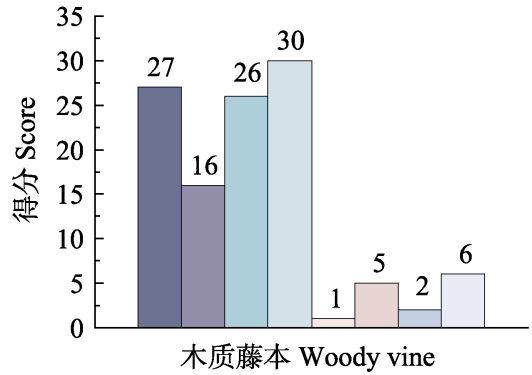

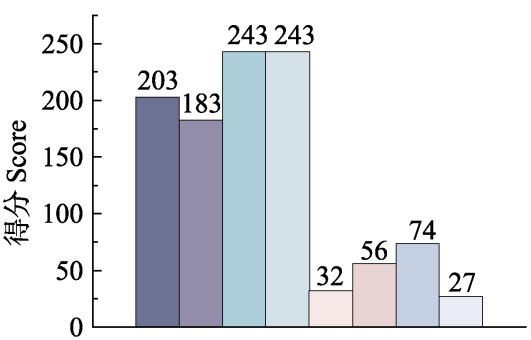

灌木 Shrub

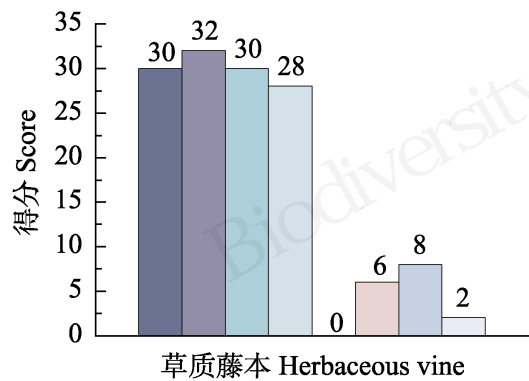

草质藤本 Herbaceous vine

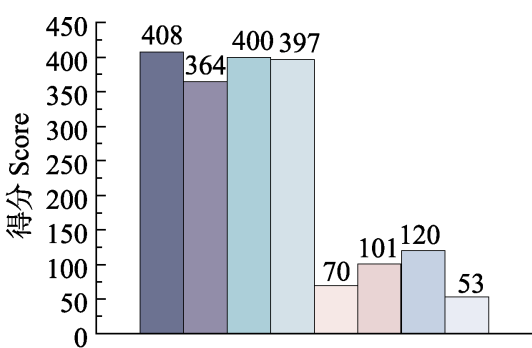

草本 Herb

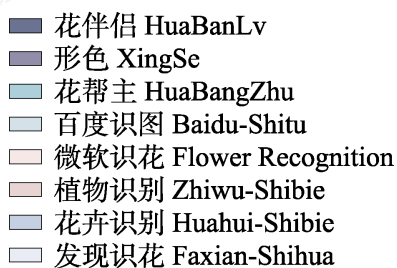

$\square$ 花卉识别 Huahui-Shibie

图3 国内8款常用植物识别软件对于不同生长型的识别结果得分

Fig. 3 Score of identification result of eight commonly used plant identification application softwares in China for different growth forms 

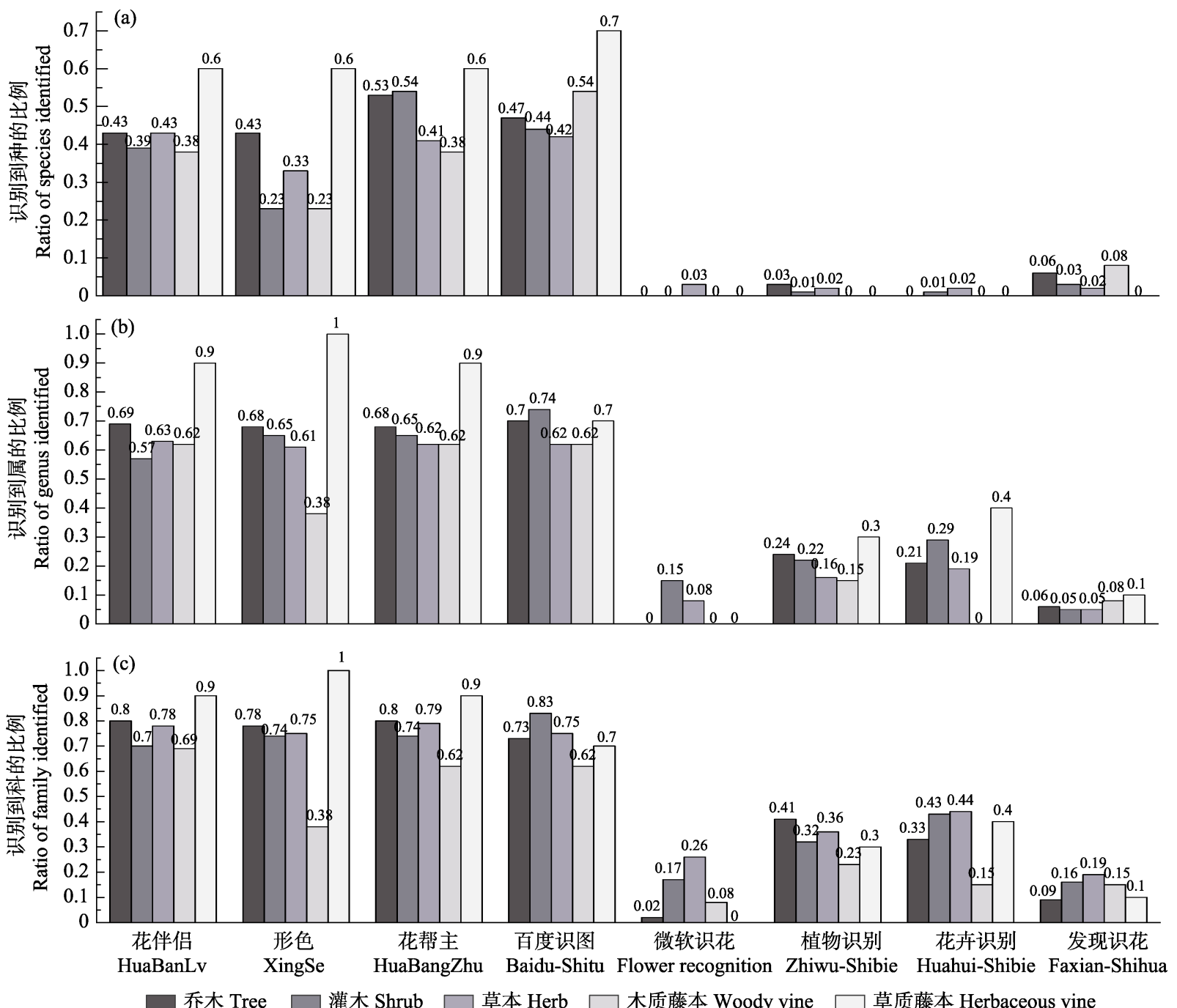

图4 国内8款常用植物识别软件对于不同生长型识别到种(a)、属(b)、科(c)的比例

Fig. 4 Identification results of eight commonly used plant identification application softwares in China for different growth forms (a) Ratio of species identified; (b) Ratio of genus identified; (c) Ratio of family identified.

别、花卉识别、发现识花的比例较低, 有比例为 $0 \%$ 的情况出现。比较乔木识别到属、科的比例, 8 款app 的差距减小, 微软识花、植物识别、花卉识别、发 现识花这 4 款app仍具备一定的识别效果, 其中植物 识别、花卉识别的比例较高。其他生长型的识别情 况与乔木的大致相同: 灌木正确识别(即识别到种) 比例最高的为花帮主(54\%); 草本为花伴侣(43\%); 木质藤本为百度识图(54\%); 草质藤本为百度识图 (70\%)(图4a)。其中, 在对草质藤本的识别中, 形色 表现也较好: 识别到种的比例为 $60 \%$, 而识别到属 和科的比例均为 $100 \%$ (图4b、c)。从图中分析, 在 本测试中, 表现较好且较稳定的app为花帮主和百 度识图。

\section{4 对国家级保护植物的识别结果}

从识别比例来分析, 花帮主对于国家一、二级 保护植物的正确识别(识别到种)比例得分最高, 花 伴侣、形色次之。对于识别到科的比例, 百度识图 最高, 为 $83 \%$, 花伴侣、形色、花帮主的比例相等。 花卉识别、植物识别、发现识花、微软识花 4 款app 的正确比例相对较低。其中, 微软识花的3项数据均 为 0 , 即科属种全部识别错误(图5)。

\section{3 讨论}

\section{1 识别结果分析}

本文所测试的 8 款app均为目前在教育、科研以 及科普方面应用较广、用户下载量较大的软件, 每 


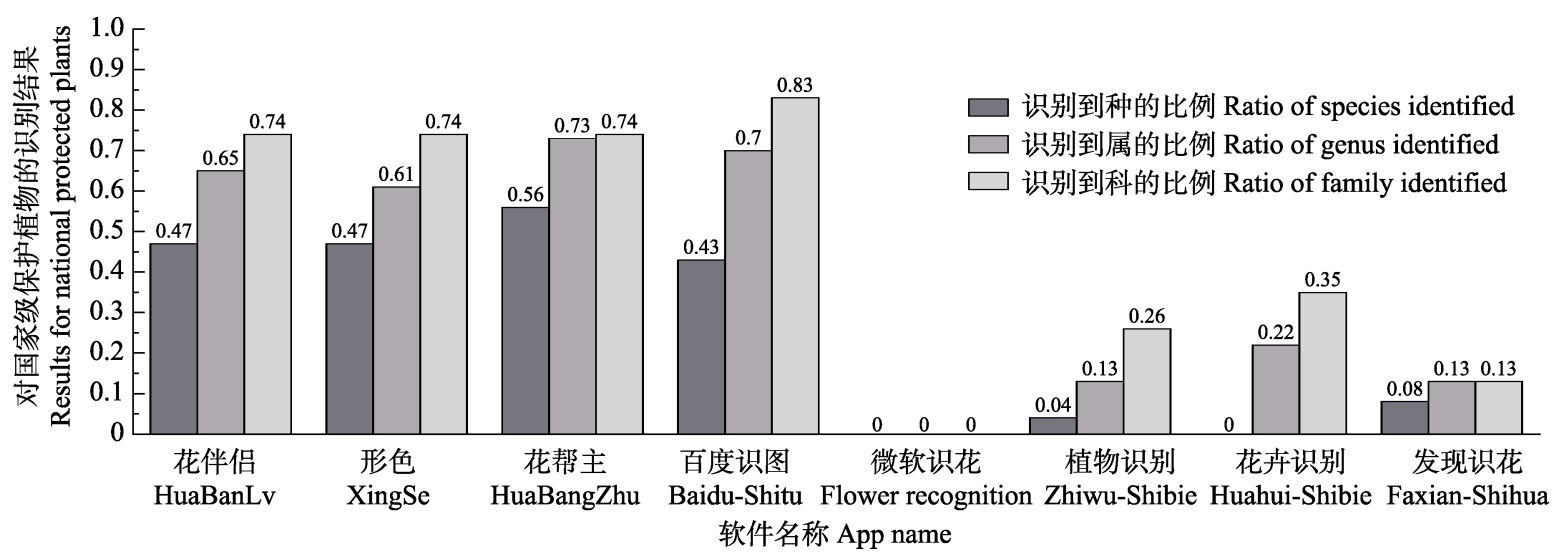

图5 国内8款常用植物识别软件对国家级保护植物(共23张图片)的识别结果

Fig. 5 Identification results of eight commonly used plant identification application softwares in China for national protected plants (23 pictures in total)

款app都具有丰富的图片资源和算法支持。对识别 软件来说, 植物分类图像库是最为核心和重要的资 源, 图像库中图片的数量和质量十分关键, 同时算 法和硬件也是重要因素之一。除此之外, 测试环境 也可以决定识别的结果, 手机品牌、系统、性能、 内存、照片背景颜色、相机分辨率、光线、拍摄角 度等等都会导致或大或小的差异(袁银等, 2018)。本 测试仅控制了手机性能、手机内存、网络环境、拍 摄角度、图片质量等因素, 故只能部分地反映各个 app的识别能力。

在本测试中, 花帮主、百度识图、花伴侣、百 度识图这4款app识别结果的准确率较高, 但对于不 同气候区、不同生活型的植物, 识别结果略有差异, 总体来说, 花帮主表现最好。值得注意的是, 智能 识别是一个自我学习和自我修正的系统, 准确率提 升依赖于数据的丰富性, 也就是说用户量越大, 数 据积累越丰富, 模型更新得越快, 系统的识别能力 也会随之提高。花帮主、百度识图、花伴侣和形色 研发较早, 占据了较大的市场份额, 用户范围也相 对较广。此外, 相对而言, 百度识图拥有比其他识 别软件更庞大的图像数据资源, 其数据资源来自整 个互联网，没有倾向性。

从 4 个气候区的得分情况来看, 干旱半干旱区 最低。根据王礼等(2018)的观点, 植物识别app的识 别系统往往依托庞大的植物数据库, 采用人工智能 识别技术, 但其植物识别过程较粗放、不精细, 可能 是因为过于庞大的数据库反而容易产生误差, 导致 识别结果不够准确。
值得注意的是, 花帮主软件本身的关注点在于 园艺花卉, 但在本次测试中其识别准确率却高于由 同一团队开发的关注点为华北野花的花伴侣，其中 原因有待探究。此外, 微软识花是唯一一款可以离 线使用的软件, 但在本次测试中准确率是 8 款中最 低的。

\section{2 植物识别app的局限性}

值得指出的是，虽然植物识别app的使用使鉴 别植物变得更简单，降低了植物分类学知识积累的 门槛, 但它们只是识别植物的一种辅助手段, 一方 面是因为植物识别app的准确率尚待提高, 另一方 面是它们始终不能取代植物学家。因为识别软件的 算法与传统的分类学、形态学的原理始终不同, 所 以对于学者来说, 扎实掌握分类学的知识和原则是 准确判断植物种类的关键。对于学生和爱好者而言, 如果没有系统扎实的分类学基础，当植物识别app 给出易混淆的识别结果的时候, 容易被误导, 也难 以纠错。

\section{4 展望}

\section{1 识别软件可应用的领域}

\subsection{1 教学}

植物识别app可在高校植物学、树木学、生态 学等一些课程教学、科研项目训练中推广。对许多 农林院校来说，校园内信息不全的挂牌、简单的记 录或查询系统已经不能满足系统学习植物分类学 的需要, 此时植物识别软件可派上用场。植物识别 app有灵活、便携、直观、反馈快速等优点, 可作为 
老师课堂教学内容的补充。在第十届应用信息学远 程学习国际科学会议上, Vesely (2014)等展示了将 新型的基于互联网的系统应用于植物学教学的成 果。经过持续22周的观察, 总结了园艺专业大学生 植物识别能力的提升情况, 研究者发现, 创建一个 可持续的基于互联网的学生教育平台是可行的。在 野外实习时, 植物识别app也可帮助学生快速且较 准确地识别植物。植物识别app还为学生利用碎片 化时间学习植物的相关知识提供了条件, 也可激发 同学们的学习兴趣, 拓宽视野。根据测评结果, 在 教学活动中, 优先推荐花帮主、百度识花、花伴侣 以及形色。

\subsection{2 科研}

植物系统分类学是其他生命科学分支的基础, 对于研究物种的适应、进化、生理、地理分布和保 护等均具有重要意义。植物识别软件可以帮助科研 工作者快速识别植物, 即便有时识别精度不够, 但 仍然是一个为科研工作者减轻负担、提高效率的方 法。在森林资源清查中, 生物多样性普查是非常重 要的一个方面。广东有 3,000 多个地块, 过去工作人 员只能通过拍摄图片请专家识别, 而植物识别软件 的应用大大提高了工作效率(王桢和何建勇, 2017)。 广东森林资源调查规划院在 2017年的森林资源连 续清查工作中使用了花伴侣。同时, 植物大数据平 台能够为生态监测、物种多样性调查、珍稀濒危植 物保护、区域物种分布、野生植物资源调查和利用 等提供数据支撑。从上述不同气候区、不同生活型 以及对国家一、二级保护植物的识别结果来看, 优 先推荐花帮主和百度识花。

\subsection{3 公众科学与科学普及}

公众科学(citizen science)是指全部或部分由业 余(或非专业)爱好者进行的科学研究。它是一种以 协同合作方式进行的科学活动, 具有开放、协作、 公共的特点。中国的公众科学方兴未艾, 而智能手 机的普及拓宽了其发展道路。

植物识别软件可作为知识传播和辅助科学研 究的重要工具。近年来, 我国公众科学素质呈现不 断上升的趋势, 从互联网上获取科技信息的公众数 量越来越多, 通过互联网传播科学信息也逐渐成为 向民众普及科学知识以及教育的重要手段 ${ }^{(1)}$ 。植物

(1) 姬洪瑜 (2015) 基于iPad平台的植物科普应用软件的设计与实现. 硕 士学位论文, 北京林业大学, 北京.
识别软件的科普及社交版块为用户快速入门提供 了可能。它能让用户熟悉身边的植物, 对自然产生 兴趣。尤其是针对珍稀濒危植物的识别, 可帮助用 户形成物种保护的意识。

数据的收集和分类是公众科学的重要内容。目 前在生物学、生态学研究中, 有大量有关鸟类、蝴 蝶、天文学、珊瑚礁的数据来自公众科学(张健等, 2013)。与此同时, 公众科学和社交网络工具的出现 促成了自然观察网络平台的诞生，如e-bird (https:// ebird.org/home), inaturalist (https://www.inaturalist.org/), tela botanica (https://www.tela-botanica.org/), 这些 公众科学网站已十分成熟并有自主开发的app。再如 xeno canto (https://www.xeno-canto.org/)和ispot (https: //www.ispotnature.org/)这两个网站则拥有成熟的植 物识别网络社区, 甚至提供在线公开课(Joly et al, 2016)。植物物种信息可以作为一个新的方向, 植物 识别软件有望成为相关领域的高质量数据的主要 来源, 为决策者和科学家提供帮助, 大量节省时 间、成本。同时，公众科学还能增加普通民众对科 学的参与感, 使其不再高不可攀。基于总体的识别 结果, 优先推荐花帮主、百度识图、花伴侣和形色。

\subsection{4 园林设计方面的应用}

在2017年北京智慧园林论坛上, 吴安东在题为 《大数据+AI图像识别技术在园林行业应用探索》 的演讲中提出, 像花伴侣这样的植物识别软件, 除 了对普通市民起到科普工具的作用外, 对于公园风 景区以及农林业的一线工作人员都能产生价值。

对于园林工作者来说, 通过植物识别软件了解 植物的开花时间、习性、分布区等信息, 可帮助他 们选择适合的园林绿化、园林造景植物。对于园林 的游览者来说，了解当季的花卉、植物的名称、寓 意、物候信息可提升园林游赏体验。普通民众不仅 可以知晓植物的物候, 还可以拍摄和上传照片/视 频, 帮助管理者掌握第一手信息(王桢, 2017)。从测 试结果和软件本身的属性来看, 优先推荐科普功能 较齐全的形色和花帮主。

\section{2 识别软件发展趋势及优化途径}

可以预见, 植物识别软件将有更多作为, 其功 能将不断完善并广泛应用于各个领域。各个识别软 件应着眼于扩大图像库的数据量、优化算法、拓展 功能、优化用户体验, 吸引更多用户。如百度的“AI 
植物园计划” 已经与 5 所国内知名植物园达成合作 意向, 并落地“百度探花郎”产品, 兼顾拍照识花、全 景科普以及智能导览三大功能。国外的iNaturalist 开发出不同语言的网络版本, 广泛搜集生物图片及 其地理分布信息, 已成为生物多样性信息学研究的 有力工具。

植物识别软件功能还可以更具针对性, 增强应 用性。如开发适合不同地区(如保护地)和专门用途 (如植物标本鉴定、花粉/咆粉鉴定)的植物识别软件; 与野外植物考察结合, 实现识别与记录同步(如中 国科学院昆明植物所研究开发的Biotracks); 与其 他生物类群识别整合, 开发综合的生物识别软件 (如中国科学院动物研究所的“生物记”)。在农业上, 可开发出识别常见病虫害的功能, 帮助农民解决难 题; 在物种保护领域, 可着眼于对珍稀、濒危和重 要资源植物的识别; 在生物安全领域，可用软件识 别有害生物, 如外来入侵种等。随着植物识别软件 功能变得更全面、识别变得更智能, 我们可以便捷 地了解身边的一草一木, 并为保护环境、发展产业 和丰富生活做出贡献。

\section{参考文献}

Cao XY, Sun WM, Zhu YX, Qian X, Li XY, Ye N (2018) Plant image recognition based on family priority strategy. Journal of Computer Application, 38(11), 195-199. (in Chinese with English abstract) [曹香漟, 孙卫民, 朱悠翔, 钱 金金, 李晓宇, 业宁 (2018) 基于科优先策略的植物图像识 别. 计算机应用, 38(11), 195-199.]

Joly A, Bonnet P, Goeau H, Barbe J, Selmi S, Champ J, Kowalski SD, Affouard A, Carre J, Molino JF, Boujemaa N, Barthelemy D (2016) A look inside the Pl@ntNet experience. Multimedia Systems, 22, 751-766.

Kumar N, Belhumeur PN, Biswas A, Jacobs DW, Kress WJ, Lopez I, Soares JVB (2012) Leafsnap: A computer vision system for automatic plant species identification. In: European Conference on Computer Vision (eds Fitzgibbon A, Lazebnik S, Perona P, Sato Y, Schmid C), pp. 502-516. Springer-Verlag, Berlin.

Li M (2018) “flower partners": A new medium of knowledge service in the era of artificial intelligence. Publishing Reference, (8), 23-24. (in Chinese) [李敏 (2018) “花伴侣”: 人 工智能时代知识服务的新媒介. 出版参考, (8), 23-24.]

Li MT, Zhang JT, Lei YH (2017) Influence analysis of Chinese plant science popularization app based on user demand-A case study of "HuabanLv" App. Science and Technology Communication, 9(22), 211-213. (in Chinese) [李珢葶, 张
健涛, 雷耀华 (2017) 基于用户需求的国内植物类科普 app影响力分析一一花伴侣App为例. 科技传播, 9(22), 211-213.]

Vesely O, Vild O, Stastny J (2014) A novel approach for the increase in student's learning motivation. In: DIVAI 2014: The 10th International Scientific Conference on Distance Learning in Applied Informatics, pp. 529-536. Wolters Kluwer, Sturovo.

Wang JX (2019) Research on the communication effect of "huajian" article on shape-color App based on "5W" communication mode. Audio-Visual, 142(2), 131-132. (in Chinese). [王加希 (2019) 基于“5W”传播模式对形色App“花间”文 章传播效果的探究. 视听, 142(2), 131-132.]

Wang L, Hong ZB, Fang LM, Chen X, Wu C (2018) Plant leaf recognition technology based on image analysis.Journal of Zhejiang A \& F University, 35, 900-907. (in Chinese with English abstract) [王礼, 洪祖兵, 方陆明, 陈珣, 吴超 (2018) 基于iOS系统的观赏植物识别. 浙江农林大学学 报, 35, 900-907.]

Wang Z, He JY (2017) "HuaBanLv": Put the encyclopedia of plants into pocket. Greening and Life, (10), 30-33. (in Chinese) [王桢，何建勇 (2017) 花伴侣: 把“植物大百科” 装进小口袋. 绿化与生活, (10), 30-33. ]

Yuan Y, Huang ZQ, Ding SF, Liu YJ, Feng ZJ (2018) Research on sampling method of plants based on big data analysis. Modern Agricultural Science and Technology, (3), 149-151. (in Chinese with English abstract) [袁银, 黄稚清, 丁释丰, 刘永金, 冯志坚 (2018) 基于大数据分析的植物标本取 样方法研究. 现代农业科技, (3), 149-151.]

Zhang J, Chen SB, Chen B, Du YJ, Huang XL, Pan XB, Zhang Q (2013) Citizen science: Integrating scientific research, ecological conservation and public participation. Biodiversity Science, 21, 738-749. (in Chinese with English abstract) [张健, 陈圣宾, 陈涁, 杜彦君, 黄晓磊, 潘绪斌, 张强 (2013) 公众科学: 整合科学研究、生态保护和公众参与. 生物多样性, 21, 738-749.]

Zhang JJ, Guo JH, Wang ZZ, Wang PB, Wu XS (2017) “Campus Plants”, Design and development of the plant-recognize software-Take Donghu campus of Zhejiang Agricultural and Forestry University as an example. South Agricultural Machinery, 48(5), 80-81. (in Chinese) [张健捷, 郭建辉, 王志真, 王拼搏, 吴小双 (2017) “校园植物通” 定位识别 软件设计与研发一以浙江农林大学东湖校区为例. 南 方农机, 48(5), 80-81.]

Zhang N, Liu WP (2011) Plant leaf recognition technology based on image analysis. Application Research of Computers, 28, 4001-4007. (in Chinese with English abstract) [张 宁, 刘文萍 (2011) 基于图像分析的植物叶片识别技术 综述. 计算机应用研究, 28, 4001-4007.]

Zhang YZ, Li BY (2016) Remote rapid recognition method for plants. Journal of Computer Applications, 36(S2), 206-209. 
(in Chinese with English abstract) [张玉玩, 李柏依 (2016) 植物远程快速识别的方法. 计算机应用, 36(S2), 206-209.]

Zhong GL, Wang Q, Li MR, Lu Y (2015) Know-It-All-A recognition system of leaves. Internet of Things Technologies, 5(12), 6-7. (in Chinese) [钟刚亮, 王强, 李
梦如, 卢遥 (2015) 百叶通—植物叶片识别系统. 物联 网技术, 5(12), 6-7.]

(责任编委：黄晓䂞 责任编辑：黄祥忠)

\section{附录 Supplementary Material}

附录1 国内8 款常用植物识别软件的主要特点比较

Appendix 1 Comparison of the main characteristics of eight commonly used plant identification application softwares in China under evaluation

http://www.biodiversity-science.net/fileup/PDF/2019272-1.pdf

附录2 测评所用的 $\mathbf{4 0 0}$ 张植物图片

Appendix 2400 pictures used for evaluation

http://www.nsii.org.cn/2017/testPhoto.php

附录3 测评所用的照片对应的植物名录

Appendix 3 A list of plants corresponding to the photographs used for the evaluation http://www.biodiversity-science.net/fileup/PDF/2019272-2.pdf

附录4 国内8款常用植物识别软件的识别结果统计表

Appendix 4 Identification results of eight commonly used plant identification application softwares in China http://www.biodiversity-science.net/fileup/PDF/2019272-3.xlsx 
许展慧, 刘诗尧, 赵芗, 涂文琴, 常诏峰, 张恩涛, 郭靖, 郑迪, 耿釷, 顾高营, 郭淳鹏, 郭璐璐, 王静, 徐春 阳, 彭钏, 杨腾, 崔梦琪, 孙伟成, 张剑坛, 刘皓天, 巴超群, 王鹤琪, 贾竞超, 武金洲, 肖翠, 马克平. 国内 8 款常用植物识别软件的识别能力评价. 生物多样性, 2020, 28 (4):524-533. http://www.biodiversityscience.net/CN/10.17520/biods.2019272

附录 1 国内8款常用植物识别软件的主要特点比较

Appendix 1 Comparison of the main characteristics of eight commonly used plant identification application softwares in China under evaluation

\begin{tabular}{|c|c|c|c|c|c|}
\hline App名称 & 基于资源 & 算法 & 硬件 & 关注点 & 可识别植物种数 \\
\hline App name & Resources based & Algorithm & Hardware & Concerns & No. of species identified \\
\hline 花伴侣 & 中国科学院植物研究所+北京鲁 & 核心算法一致 & 中国科学院植物 & 华北野花 & 11,000 种 \\
\hline HuaBanLv & 朗公司 & & 研究所服务器 & & \\
\hline 形色 & 中国科学院植物研究所+杭州大 & 核心算法一致 & 公司服务器 & 所有植物 & 4,000种 \\
\hline XingSe & 拿公司 & & & & \\
\hline 花帮主 & 中国科学院植物研究所+北京鲁 & 核心算法一致 & 公司服务器 & 园艺 & 未公开 \\
\hline HuaBangZhu & 朗公司 & & & & \\
\hline 百度识图 & 中国科学院植物研究所专家清 & 互联网公开的核心算法 & 最为强大 & 所有植物 & 未公开 \\
\hline \multirow[t]{2}{*}{ Baidu-Shitu } & 洗数据+大量互联网信息 & +专家清洗数据(整个互 & & & \\
\hline & & 联网资源) & & & \\
\hline 微软识花 & 个别专家图库 & 核心算法一致 & 公司服务器 & 花卉 & 未公开 \\
\hline \multicolumn{6}{|c|}{ Flower recognition } \\
\hline 植物识别 & 未知 & 核心算法一致 & 公司服务器 & 所有植物 & 未公开 \\
\hline \multicolumn{6}{|l|}{ Zhiwu-Shibie } \\
\hline 花卉识别 & 网络资源 & 核心算法一致 & 公司服务器 & 花卉 & 未公开 \\
\hline \multicolumn{6}{|l|}{ Huahui-Shibie } \\
\hline 发现识花 & 网络资源 & 核心算法一致 & 公司服务器 & 花卉 & 未公开 \\
\hline Faxian-Shihua & & & & & \\
\hline
\end{tabular}


许展慧, 刘诗尧, 赵莹, 涂文琴, 常诏峰, 张恩涛, 郭靖, 郑迪, 耿均, 顾高营, 郭淳鹏, 郭璐璐, 王静, 徐春 阳, 彭钏, 杨腾, 崔梦琪, 孙伟成, 张剑坛, 刘皓天, 巴超群, 王鹤琪, 贾竞超, 武金洲, 肖翠, 马克平. 国内 8 款常用植物识别软件的识别能力评价. 生物多样性, 2020, 28 (4):524-533. http://www.biodiversityscience.net/CN/10.17520/biods.2019272

附录3 测评所用的照片对应的植物名录

Appendix 3 A list of plants corresponding to the photographs used for the evaluation

\begin{tabular}{|c|c|}
\hline 物种 & 照片在 NSII 网页的地址 \\
\hline \multicolumn{2}{|l|}{ 一、干旱半干旱区 } \\
\hline \multicolumn{2}{|l|}{ 1.麻黄科 Ephedraceae } \\
\hline 1.蓝枝麻黄 Ephedra glauca & $\begin{array}{l}\text { http://www.nsii.org.cn/nsiiresources/media/photo/testPhoto/1d/original/蓝枝麻黄（果实）（Ephedra glauca） } \\
\text { DSC_0065.jpg }\end{array}$ \\
\hline 3.草麻黄 Ephedra sinica & $\begin{array}{l}\text { http://www.nsii.org.cn/nsiiresources/media/photo/testPhoto/1d/original/草麻黄（Ephedra sinica）段士民 } \\
\text { DSC_0044.jpg }\end{array}$ \\
\hline \multicolumn{2}{|l|}{ 2.杨柳科 Salicaceae } \\
\hline \multicolumn{2}{|l|}{ 3.蓼科 Polygonaceae } \\
\hline \multicolumn{2}{|l|}{ 1.木蓼属 Atraphaxis } \\
\hline 1.长枝木蓼 Atraphaxis virgata & http://www.nsii.org.cn/nsiiresources/media/photo/testPhoto/1d/original/长枝木蓼（Atraphaxis virgata）段士民.jpg \\
\hline \multicolumn{2}{|l|}{ 4.石竹科 Caryophyllaceae } \\
\hline \multicolumn{2}{|l|}{ 1.刺叶属 Acanthophyllum } \\
\hline 1.刺叶 Acanthophyllum pungens & $\begin{array}{l}\text { http://www.nsii.org.cn/nsiiresources/media/photo/testPhoto/1d/original/刺叶（Acanthophyllum pungens）段士 } \\
\text { 民.jpg }\end{array}$ \\
\hline \multicolumn{2}{|l|}{ 2.蝇子草属 Silene } \\
\hline \multicolumn{2}{|l|}{ 2.驼线揫属 Ceratoides } \\
\hline 1.驼线藜 Ceratoides latens & $\begin{array}{l}\text { http://www.nsii.org.cn/nsiiresources/media/photo/testPhoto/1d/original/驼线傣（Ceratoides latens）段士民 } \\
\text { DSC_0013.jpg }\end{array}$ \\
\hline \multicolumn{2}{|l|}{ 3.角果藜属 Ceratocarpus } \\
\hline 1.角果藜 Ceratocarpus arenarius & $\begin{array}{l}\text { http://www.nsii.org.cn/nsiiresources/media/photo/testPhoto/1d/original/角果藜（Ceratocarpus arenarius）段士民 } \\
\text { DSC_0038.jpg }\end{array}$ \\
\hline \multicolumn{2}{|l|}{ 4.菉属 Chenopodium } \\
\hline 1.球花藜 Chenopodium foliosum & $\begin{array}{l}\text { http://www.nsii.org.cn/nsiiresources/media/photo/testPhoto/1d/original/球花葱（Chenopodium foliosum）段士民 } \\
\text { DSC_2612.jpg }\end{array}$ \\
\hline \multicolumn{2}{|l|}{ 5.盐生草属 Halogeton } \\
\hline 1.盐生草 Halogeton glomeratus & $\begin{array}{l}\text { http://www.nsii.org.cn/nsiiresources/media/photo/testPhoto/1d/original/盐生草（Halogeton glomeratus）段士 } \\
\text { 民 DSC_0157.jpg }\end{array}$ \\
\hline 6.梭梭属 Haloxylon & 1) \\
\hline
\end{tabular}


许展慧, 刘诗尧, 赵芗, 涂文琴, 常诏峰, 张恩涛, 郭靖, 郑迪, 耿釷, 顾高营, 郭淳鹏, 郭璐璐, 王静, 徐春 阳, 彭钏, 杨腾, 崔梦琪, 孙伟成, 张剑坛, 刘皓天, 巴超群, 王鹤琪, 贾竞超, 武金洲, 肖翠, 马克平. 国内 8 款常用植物识别软件的识别能力评价. 生物多样性, 2020, 28 (4):524-533. http://www.biodiversityscience.net/CN/10.17520/biods.2019272

\begin{tabular}{|c|c|}
\hline 物种 & 照片在 NSII 网页的地址 \\
\hline 1.无叶梭梭 Haloxylon aphyllum & $\begin{array}{l}\text { http://www.nsii.org.cn/nsiiresources/media/photo/testPhoto/1d/original/无叶梭梭（果实）(Haloxylon aphyllum）- } \\
\text { 王喜勇.jpg }\end{array}$ \\
\hline 2.白梭梭 Haloxylon persicum & $\begin{array}{l}\text { http://www.nsii.org.cn/nsiiresources/media/photo/testPhoto/1d/original/白梭梭（Haloxylon persicum）果实, 新疆 } \\
\text { 精河县.jpg }\end{array}$ \\
\hline \multicolumn{2}{|l|}{ 7.戈壁藜属 Iljinia } \\
\hline 1.戈壁藜 Iljinia regelii & http://www.nsii.org.cn/nsiiresources/media/photo/testPhoto/1d/original/戈壁藜（Iljinia regelii）段士民.jpg \\
\hline \multicolumn{2}{|l|}{ 8.盐角草属 Salicornia } \\
\hline 1.盐角草 Salicornia europaea & http://www.nsii.org.cn/nsiiresources/media/photo/testPhoto/1d/original/盐角草（Salicornia europaea）段士民.jpg \\
\hline \multicolumn{2}{|l|}{ 9.猪毛菜属 Salsola } \\
\hline 1.散枝猪毛菜 Salsola brachiata & http://www.nsii.org.cn/nsiiresources/media/photo/testPhoto/1d/original/散枝猪毛菜（Salsola brachiata）段士民.jpg \\
\hline 2.浆果猪毛菜 Salsola foliosa & http://www.nsii.org.cn/nsiiresources/media/photo/testPhoto/1d/original/浆果猪毛菜（Salsola foliosa）段士民.jpg \\
\hline \multicolumn{2}{|l|}{ 6.毛茛科 Ranunculaceae } \\
\hline \multicolumn{2}{|l|}{ 1.角果毛莨属 Ceratocephala } \\
\hline 1.角果毛莨 Ceratocephala testiculata & $\begin{array}{l}\text { http://www.nsii.org.cn/nsiiresources/media/photo/testPhoto/1d/original/角果毛茛（Ceratocephala testiculata）段士 } \\
\text { 民 DSC_0184.jpg }\end{array}$ \\
\hline \multicolumn{2}{|l|}{ 2.铁线莲属 Clematis } \\
\hline 1.甘青铁线莲 Clematis tangutica & $\begin{array}{l}\text { http://www.nsii.org.cn/nsiiresources/media/photo/testPhoto/1d/original/甘青铁线莲（Clematis tangutica）段士民 } \\
\text { DSC_0058.jpg }\end{array}$ \\
\hline \multicolumn{2}{|l|}{ 7.罂粟科 Papaveraceae } \\
\hline \multicolumn{2}{|l|}{ 1.海罂粟属 Glaucium } \\
\hline 1.鳞果海罂粟 Glaucium squamigerum & $\begin{array}{l}\text { http://www.nsii.org.cn/nsiiresources/media/photo/testPhoto/1d/original/鳞果海罂粟（Glaucium squamigerum）段士 } \\
\text { 民.jpg }\end{array}$ \\
\hline \multicolumn{2}{|l|}{ 8.十字花科 Cruciferae } \\
\hline \multicolumn{2}{|l|}{ 1.群心菜属 Cardaria } \\
\hline 毛果群心菜 Cardaria pubescens & $\begin{array}{l}\text { http://www.nsii.org.cn/nsiiresources/media/photo/testPhoto/1d/original/毛果群心菜（Cardaria pubescens）段士民 } \\
\text { DSC_0015.jpg }\end{array}$ \\
\hline \multicolumn{2}{|l|}{ 2.涩荠属 Malcolmia } \\
\hline 1.澀荠 Malcolmia africana & $\begin{array}{l}\text { http://www.nsii.org.cn/nsiiresources/media/photo/testPhoto/1d/original/澀荠（Malcolmia africana）, 新疆乌鲁木 } \\
\text { 齐.jpg }\end{array}$ \\
\hline \multicolumn{2}{|l|}{ 3.菥暻属 Thlaspi } \\
\hline 1.菥营 Thlaspi arvense & $\begin{array}{l}\text { http://www.nsii.org.cn/nsiiresources/media/photo/testPhoto/1d/original/菥营（Thlaspi arvense），段士民 } \\
\text { DSC_0026.jpg }\end{array}$ \\
\hline \multicolumn{2}{|l|}{ 9.薔微科 Rosaceae } \\
\hline \multicolumn{2}{|l|}{ 1.蔷薇属 Hulthemia } \\
\hline 1.单叶菩薇 Hulthemia berberifolia & $\begin{array}{l}\text { http://www.nsii.org.cn/nsiiresources/media/photo/testPhoto/1d/original/单叶蔷薇（Hulthemia berberifolia），段士 } \\
\text { 民.jpg }\end{array}$ \\
\hline \multicolumn{2}{|l|}{ 2.绣线菊属 Spiraea } \\
\hline $\begin{array}{l}\text { 1.金丝桃叶绣线菊 Spiraea } \\
\text { hypericifolia }\end{array}$ & $\begin{array}{l}\text { http://www.nsii.org.cn/nsiiresources/media/photo/testPhoto/1d/original/金丝桃叶绣线菊（Spiraea hypericifolia）段 } \\
\text { 士民 DSC_0202.jpg }\end{array}$ \\
\hline \multicolumn{2}{|l|}{ 10.豆科 Fabaceae } \\
\hline 1.骆驼刺属 Alhagi & \\
\hline
\end{tabular}


许展慧, 刘诗尧, 赵莹, 涂文琴, 常诏峰, 张恩涛, 郭靖, 郑迪, 耿均, 顾高营, 郭淳鹏, 郭璐璐, 王静, 徐春 阳, 彭钏, 杨腾, 崔梦琪, 孙伟成, 张剑坛, 刘皓天, 巴超群, 王鹤琪, 贾竞超, 武金洲, 肖翠, 马克平. 国内 8 款常用植物识别软件的识别能力评价. 生物多样性, 2020, 28 (4):524-533. http://www.biodiversityscience.net/CN/10.17520/biods.2019272

\begin{tabular}{|c|c|}
\hline 物种 & 照片在 NSII 网页的地址 \\
\hline 1.骆驼刺 Alhagi sparsifolia & $\begin{array}{l}\text { http://www.nsii.org.cn/nsiiresources/media/photo/testPhoto/1d/original/骆驼刺（Alhagi sparsifolia）段士民 } \\
\text { IMG_8534.jpg }\end{array}$ \\
\hline \multicolumn{2}{|l|}{ 2.沙冬青属 Ammopiptanthus } \\
\hline 1.沙冬青 Ammopiptanthus mongolicus & $\begin{array}{l}\text { http://www.nsii.org.cn/nsiiresources/media/photo/testPhoto/1d/original/沙冬青（Ammopiptanthus mongolicus）段 } \\
\text { 士民 DSC_0083.jpg }\end{array}$ \\
\hline 2.小沙冬青 Ammopiptanthus nanus & $\begin{array}{l}\text { http://www.nsii.org.cn/nsiiresources/media/photo/testPhoto/1d/original/小沙冬青（Ammopiptanthus nanus）段士民 } \\
\text { DSC_0002.jpg }\end{array}$ \\
\hline \multicolumn{2}{|l|}{ 3.黄耆属 Astragalus } \\
\hline 1.长毛荚黄耆 Astragalus macrotrichus & $\begin{array}{l}\text { http://www.nsii.org.cn/nsiiresources/media/photo/testPhoto/1d/original/长毛荚黄耆（Astragalus macrotrichus）段 } \\
\text { 士民.jpg }\end{array}$ \\
\hline 2.茧荚黄耆 Astragalus lehmannianus & $\begin{array}{l}\text { http://www.nsii.org.cn/nsiiresources/media/photo/testPhoto/1d/original/茧荚黄耆（Astragalus lehmannianus）段士 } \\
\text { 民.jpg }\end{array}$ \\
\hline \multicolumn{2}{|l|}{ 4.无叶豆属 Eremosparton } \\
\hline $\begin{array}{l}\text { 1.准噶尔无叶豆 Eremosparton } \\
\text { songoricum }\end{array}$ & $\begin{array}{l}\text { http://www.nsii.org.cn/nsiiresources/media/photo/testPhoto/1d/original/准噶尔无叶豆 (Eremosparton songoricum) } \\
\text { 段士民 DSC_0062.jpg }\end{array}$ \\
\hline \multicolumn{2}{|l|}{ 5.锦鸡儿属 Caragana } \\
\hline $\begin{array}{l}\text { 1.刺叶锦鸡儿 Caragana } \\
\text { acanthophylla }\end{array}$ & $\begin{array}{l}\text { http://www.nsii.org.cn/nsiiresources/media/photo/testPhoto/1d/original/刺叶锦鸡儿（Caragana acanthophylla）段士 } \\
\text { 民 DSC_0123.jpg }\end{array}$ \\
\hline \multicolumn{2}{|l|}{ 6.甘草属 Glycyrrhiza } \\
\hline 1.粗毛甘草 Glycyrrhiza aspera & http://www.nsii.org.cn/nsiiresources/media/photo/testPhoto/1d/original/粗毛甘草（Glycyrrhiza aspera）段士民.jpg \\
\hline 2.胀果甘草 Glycyrrhiza inflata & $\begin{array}{l}\text { http://www.nsii.org.cn/nsiiresources/media/photo/testPhoto/1d/original/胀果甘草（Glycyrrhiza inflata）段士民 } \\
\text { DSC_0072.jpg }\end{array}$ \\
\hline 3.甘草 Glycyrrhiza uralensis & $\begin{array}{l}\text { http://www.nsii.org.cn/nsiiresources/media/photo/testPhoto/1d/original/甘草（Glycyrrhiza uralensis），新疆青河 } \\
\text { 县.jpg }\end{array}$ \\
\hline \multicolumn{2}{|l|}{ 7.铃铛刺属 Halimodendron } \\
\hline 1.铃铛刺 Halimodendron halodendron & $\begin{array}{l}\text { http://www.nsii.org.cn/nsiiresources/media/photo/testPhoto/1d/original/铃铛刺 (Halimodendron halodendron ) 段 } \\
\text { 士民.jpg }\end{array}$ \\
\hline \multicolumn{2}{|l|}{ 8.岩黄耆属 Hedysarum } \\
\hline 1.细枝岩黄耆 Hedysarum scoparium & $\begin{array}{l}\text { http://www.nsii.org.cn/nsiiresources/media/photo/testPhoto/1d/original/细枝岩黄耆（Hedysarum scoparium）段士 } \\
\text { 民.jpg }\end{array}$ \\
\hline \multicolumn{2}{|l|}{ 9.苦马豆属 Sphaerophysa } \\
\hline 1.苦马豆 Sphaerophysa salsula & $\begin{array}{l}\text { http://www.nsii.org.cn/nsiiresources/media/photo/testPhoto/1d/original/苦马豆（Sphaerophysa salsula）段士民 } \\
\text { DSC_0084.jpg }\end{array}$ \\
\hline \multicolumn{2}{|l|}{ 11.蒺藜科 Zygophyllaceae } \\
\hline \multicolumn{2}{|l|}{ 1.白刺属 Nitraria } \\
\hline 1.泡果白刺 Nitraria sphaerocarpa & $\begin{array}{l}\text { http://www.nsii.org.cn/nsiiresources/media/photo/testPhoto/1d/original/泡果白刺（Nitraria sphaerocarpa）段士民 } \\
\text { DSC_0046.jpg }\end{array}$ \\
\hline 2.唐古特白刺 Nitraria tangutorum & $\begin{array}{l}\text { http://www.nsii.org.cn/nsiiresources/media/photo/testPhoto/1d/original/唐古特白刺（Nitraria tangutorum ）段士民 } \\
\text { DSC_0054.jpg }\end{array}$ \\
\hline \multicolumn{2}{|l|}{ 2.骆驼蓬属 Peganum } \\
\hline 1.骆驼蓬 Peganum harmala & $\begin{array}{l}\text { http://www.nsii.org.cn/nsiiresources/media/photo/testPhoto/1d/original/骆驼蓬（Peganum harmala）段士民 } \\
\text { DSC_0066.jpg }\end{array}$ \\
\hline
\end{tabular}


许展慧, 刘诗尧, 赵芗, 涂文琴, 常诏峰, 张恩涛, 郭靖, 郑迪, 耿釷, 顾高营, 郭淳鹏, 郭璐璐, 王静, 徐春 阳, 彭钏, 杨腾, 崔梦琪, 孙伟成, 张剑坛, 刘皓天, 巴超群, 王鹤琪, 贾竞超, 武金洲, 肖翠, 马克平. 国内 8 款常用植物识别软件的识别能力评价. 生物多样性, 2020, 28 (4):524-533. http://www.biodiversityscience.net/CN/10.17520/biods.2019272

\begin{tabular}{|c|c|}
\hline 物种 & 照片在 NSII 网页的地址 \\
\hline \multicolumn{2}{|l|}{ 3.驼蹄瓣属 Zygophyllum } \\
\hline 1.马它蹄瓣 Zygophyllum fabago & http://www.nsii.org.cn/nsiiresources/media/photo/testPhoto/1d/original/驼蹄瓣（Zygophyllum fabago）段士民.jpg \\
\hline 2.大花霸王 Zygophyllum potaninii & $\begin{array}{l}\text { http://www.nsii.org.cn/nsiiresources/media/photo/testPhoto/1d/original/大花霸王（Zygophyllum potaninii）段士 } \\
\text { 民 DSC_0077.jpg }\end{array}$ \\
\hline \multicolumn{2}{|l|}{ 12.亚麻科 Linaceae } \\
\hline \multicolumn{2}{|l|}{ 1.亚麻属 Linum } \\
\hline 1.亚麻 Linum usitatissimum & http://www.nsii.org.cn/nsiiresources/media/photo/testPhoto/1d/original/亚麻（Linum usitatissimum ）-王喜勇.jpg \\
\hline \multicolumn{2}{|l|}{ 13.胡颓子科 Elaeagnaceae } \\
\hline \multicolumn{2}{|l|}{ 1.胡颓子属 Elaeagnus } \\
\hline 1.尖果沙菄 Elaeagnus oxycarpa & http://www.nsii.org.cn/nsiiresources/media/photo/testPhoto/1d/original/尖果沙本（Elaeagnus oxycarpa）段士民.jpg \\
\hline \multicolumn{2}{|l|}{ 2.沙棘属 Hippophae } \\
\hline 1.沙棘 Hippophae rhamnoides & http://www.nsii.org.cn/nsiiresources/media/photo/testPhoto/1d/original/沙棘（Hippophae rhamnoides）段士民.jpg \\
\hline \multicolumn{2}{|l|}{ 14.半日花科 Cistaceae } \\
\hline \multicolumn{2}{|l|}{ 1.半日花属 Helianthemum } \\
\hline 1.半日花 Helianthemum songaricum & $\begin{array}{l}\text { http://www.nsii.org.cn/nsiiresources/media/photo/testPhoto/1d/original/半日花（Helianthemum songaricum）段士 } \\
\text { 民, 新疆伊犁.jpg }\end{array}$ \\
\hline \multicolumn{2}{|l|}{ 15.柽柳科 Tamaricaceae } \\
\hline \multicolumn{2}{|l|}{ 1.柽柳属 Tamarix } \\
\hline 1.短穗柽柳 Tamarix laxa & $\begin{array}{l}\text { http://www.nsii.org.cn/nsiiresources/media/photo/testPhoto/1d/original/短穗柽柳（Tamarix laxa）段士民 } \\
\text { DSC_0014.jpg }\end{array}$ \\
\hline 2.细穗柽柳 Tamarix leptostachys & $\begin{array}{l}\text { http://www.nsii.org.cn/nsiiresources/media/photo/testPhoto/1d/original/细穗柽柳（Tamarix leptostachys）段士民 } \\
\text { DSC_0013.jpg }\end{array}$ \\
\hline 3.多枝柽柳 Tamarix ramosissima & $\begin{array}{l}\text { http://www.nsii.org.cn/nsiiresources/media/photo/testPhoto/1d/original/多枝柽柳（Tamarix ramosissima）段士 } \\
\text { 民.jpg }\end{array}$ \\
\hline \multicolumn{2}{|l|}{ 16.锁阳科 Cynomoriaceae } \\
\hline \multicolumn{2}{|l|}{ 1.锁阳属 Cynomorium } \\
\hline 1.锁阳 Cynomorium songaricum & $\begin{array}{l}\text { http://www.nsii.org.cn/nsiiresources/media/photo/testPhoto/1d/original/锁阳（Cynomorium songaricum）段士民 } \\
\text { DSC_0018.jpg }\end{array}$ \\
\hline \multicolumn{2}{|l|}{ 17.锦葵科 Malvaceae } \\
\hline \multicolumn{2}{|l|}{ 1.木槿属 Hibiscus } \\
\hline 1.野西瓜苗 Hibiscus trionum & $\begin{array}{l}\text { http://www.nsii.org.cn/nsiiresources/media/photo/testPhoto/1d/original/野西瓜苗（Hibiscus trionum）段士民 } \\
\text { DSC_0043.jpg }\end{array}$ \\
\hline \multicolumn{2}{|l|}{ 18.白花丹科 Plumbaginaceae } \\
\hline \multicolumn{2}{|l|}{ 1.补血草属 Limonium } \\
\hline 1.族枝补血草 Limonium chrysocomum & $\begin{array}{l}\text { http://www.nsii.org.cn/nsiiresources/media/photo/testPhoto/1d/original/簇枝补血草（Limonium chrysocomum）段 } \\
\text { 士民 DSC_0083.jpg }\end{array}$ \\
\hline \multicolumn{2}{|l|}{ 2.鸡娃草属 Plumbagella } \\
\hline 1.鸡娃草 Plumbagella micrantha & $\begin{array}{l}\text { http://www.nsii.org.cn/nsiiresources/media/photo/testPhoto/1d/original/鸡娃草（Plumbagella micrantha），新疆乌 } \\
\text { 鲁木齐.jpg }\end{array}$ \\
\hline \multicolumn{2}{|l|}{ 3.驼舌草属 Goniolimon } \\
\hline 1.驼舌草 Goniolimon speciosum & $\begin{array}{l}\text { http://www.nsii.org.cn/nsiiresources/media/photo/testPhoto/1d/original/驼舌草（Goniolimon speciosum）段士民 } \\
\text { DSC_0167.jpg }\end{array}$ \\
\hline
\end{tabular}


许展慧, 刘诗尧, 赵莹, 涂文琴, 常诏峰, 张恩涛, 郭靖, 郑迪, 耿均, 顾高营, 郭淳鹏, 郭璐璐, 王静, 徐春 阳, 彭钏, 杨腾, 崔梦琪, 孙伟成, 张剑坛, 刘皓天, 巴超群, 王鹤琪, 贾竞超, 武金洲, 肖翠, 马克平. 国内 8 款常用植物识别软件的识别能力评价. 生物多样性, 2020, 28 (4):524-533. http://www.biodiversityscience.net/CN/10.17520/biods.2019272

\begin{tabular}{|c|c|}
\hline 物种 & 照片在 NSII 网页的地址 \\
\hline \multicolumn{2}{|l|}{ 19.夹竹桃科 Apocynaceae } \\
\hline \multicolumn{2}{|l|}{ 1.白麻属 Poacynum } \\
\hline 1.大叶白麻 Poacynum hendersonii & $\begin{array}{l}\text { http://www.nsii.org.cn/nsiiresources/media/photo/testPhoto/1d/original/大叶白麻（Poacynum hendersonii）段士 } \\
\text { 民.jpg }\end{array}$ \\
\hline \multicolumn{2}{|l|}{ 20.旋花科 Convolvulaceae } \\
\hline \multicolumn{2}{|l|}{ 1.旋花属 Convolvulus } \\
\hline 1.田旋花 Convolvulus arvensis & http://www.nsii.org.cn/nsiiresources/media/photo/testPhoto/1d/original/田旋花（Convolvulus arvensis）段士民.jpg \\
\hline \multicolumn{2}{|l|}{ 2.菟丝子属 Cuscuta } \\
\hline 1.南方菟丝子 Cuscuta australis & $\begin{array}{l}\text { http://www.nsii.org.cn/nsiiresources/media/photo/testPhoto/1d/original/南方菟丝子（Cuscuta australis）段士民 } \\
\text { DSC_0034.jpg }\end{array}$ \\
\hline \multicolumn{2}{|l|}{ 21.紫草科 Boraginaceae } \\
\hline \multicolumn{2}{|l|}{ 1.软紫草属 Arnebia } \\
\hline 1.黄花软紫草 Arnebia guttata & http://www.nsii.org.cn/nsiiresources/media/photo/testPhoto/1d/original/黄花软紫草（Arnebia guttata）段士民.jpg \\
\hline \multicolumn{2}{|l|}{ 2.蓝薊属 Echium } \\
\hline 1.蓝薊 Echium vulgare & $\begin{array}{l}\text { http://www.nsii.org.cn/nsiiresources/media/photo/testPhoto/1d/original/蓝蓟（Echium vulgare）段士民 } \\
\text { DSC_0004.jpg }\end{array}$ \\
\hline \multicolumn{2}{|l|}{ 3.长柱琉璃草属 Lindelofia } \\
\hline 1.长柱琉璃草 Lindelofia stylosa & $\begin{array}{l}\text { http://www.nsii.org.cn/nsiiresources/media/photo/testPhoto/1d/original/长柱琉璃草（Lindelofia stylosa）段士 } \\
\text { 民.jpg }\end{array}$ \\
\hline \multicolumn{2}{|l|}{ 4.假狼紫草属 Nonea } \\
\hline 1.假狼紫草 Nonea caspica & $\begin{array}{l}\text { http://www.nsii.org.cn/nsiiresources/media/photo/testPhoto/1d/original/假狼紫草（Nonea caspica）段士民 } \\
\text { DSC_0130.jpg }\end{array}$ \\
\hline \multicolumn{2}{|l|}{ 22.唇形科 Labiatae } \\
\hline \multicolumn{2}{|l|}{ 1.沙穗属 Eremostachys } \\
\hline 1.沙穗 Eremostachys moluccelloides & $\begin{array}{l}\text { http://www.nsii.org.cn/nsiiresources/media/photo/testPhoto/1d/original/沙穗（Eremostachys moluccelloides）段士 } \\
\text { 民.jpg }\end{array}$ \\
\hline \multicolumn{2}{|l|}{ 2.鼠尾草属 Salvia } \\
\hline 1.新疆鼠尾草 Salvia deserta & $\begin{array}{l}\text { http://www.nsii.org.cn/nsiiresources/media/photo/testPhoto/1d/original/新疆鼠尾草（Salvia deserta）段士民 } \\
\text { DSC_0138.jpg }\end{array}$ \\
\hline \multicolumn{2}{|l|}{ 3.黄芩属 Scutellaria } \\
\hline 1.盔状黄芩 Scutellaria galericulata & $\begin{array}{l}\text { http://www.nsii.org.cn/nsiiresources/media/photo/testPhoto/1d/original/盔状黄芩（Scutellaria galericulata）段士民 } \\
\text { DSC_0041.jpg }\end{array}$ \\
\hline \multicolumn{2}{|l|}{ 4.新塔花属 Ziziphora } \\
\hline 1.小新塔花 Ziziphora tenuior & $\begin{array}{l}\text { http://www.nsii.org.cn/nsiiresources/media/photo/testPhoto/1d/original/小新塔花（Ziziphora tenuior）, 新疆玛纳斯 } \\
\text { 县.jpg }\end{array}$ \\
\hline \multicolumn{2}{|l|}{ 23.茄科 Solanaceae } \\
\hline \multicolumn{2}{|l|}{ 1.茄属 Solanum } \\
\hline 1.红果龙葵 Solanum villosum & http://www.nsii.org.cn/nsiiresources/media/photo/testPhoto/1d/original/红果龙葵（Solanum villosum）段士民.jpg \\
\hline \multicolumn{2}{|l|}{ 2.天仙子属 Hyoscyamus } \\
\hline 1.天仙子 Hyoscyamus niger & http://www.nsii.org.cn/nsiiresources/media/photo/testPhoto/1d/original/天仙子（Hyoscyamus niger）段士民.jpg \\
\hline 3.枸杞属 Lycium & \\
\hline
\end{tabular}


许展慧, 刘诗尧, 赵芗, 涂文琴, 常诏峰, 张恩涛, 郭靖, 郑迪, 耿釷, 顾高营, 郭淳鹏, 郭璐璐, 王静, 徐春 阳, 彭钏, 杨腾, 崔梦琪, 孙伟成, 张剑坛, 刘皓天, 巴超群, 王鹤琪, 贾竞超, 武金洲, 肖翠, 马克平. 国内 8 款常用植物识别软件的识别能力评价. 生物多样性, 2020, 28 (4):524-533. http://www.biodiversityscience.net/CN/10.17520/biods.2019272

\begin{tabular}{|c|c|}
\hline 物种 & 照片在 NSII 网页的地址 \\
\hline 1.宁夏枸杞 Lycium barbarum & $\begin{array}{l}\text { http://www.nsii.org.cn/nsiiresources/media/photo/testPhoto/1d/original/宁夏枸杞（Lycium barbarum）段士民 } \\
\text { DSC_0275.jpg }\end{array}$ \\
\hline 2.黑果枸杞 Lycium ruthenicum & $\begin{array}{l}\text { http://www.nsii.org.cn/nsiiresources/media/photo/testPhoto/1d/original/黑果枸杞（Lycium ruthenicum）段士民 } \\
\text { DSC_0008.jpg }\end{array}$ \\
\hline \multicolumn{2}{|l|}{ 4.泡囊草属 Physochlaina } \\
\hline 1.泡囊草 Physochlaina physaloides & $\begin{array}{l}\text { http://www.nsii.org.cn/nsiiresources/media/photo/testPhoto/1d/original/泡囊草（Physochlaina physaloides）段士 } \\
\text { 民.jpg }\end{array}$ \\
\hline \multicolumn{2}{|l|}{ 24.玄参科 Scrophulariaceae } \\
\hline \multicolumn{2}{|l|}{ 1.毛芯花属 Verbascum } \\
\hline $\begin{array}{l}\text { 1.准噶尔毛芯花 Verbascum } \\
\text { songaricum }\end{array}$ & $\begin{array}{l}\text { http://www.nsii.org.cn/nsiiresources/media/photo/testPhoto/1d/original/准噶尔毛芯花（Verbascum songaricum）段 } \\
\text { 士民 DSC_0112.jpg }\end{array}$ \\
\hline \multicolumn{2}{|l|}{ 25.列当科 Orobanchaceae } \\
\hline \multicolumn{2}{|l|}{ 1.黄筒花属 Phacellanthus } \\
\hline 1.管花肉苁蓉 Cistanche tubulosa & $\begin{array}{l}\text { http://www.nsii.org.cn/nsiiresources/media/photo/testPhoto/1d/original/管花肉苁蓉（Cistanche tubulosa）段士 } \\
\text { 民.jpg }\end{array}$ \\
\hline \multicolumn{2}{|l|}{ 26.车前科 Plantaginaceae } \\
\hline \multicolumn{2}{|l|}{ 1.车前属 Plantago } \\
\hline 1.小车前 Plantago minuta & $\begin{array}{l}\text { http://www.nsii.org.cn/nsiiresources/media/photo/testPhoto/1d/original/小车前（Plantago minuta）, 新疆巴里坤 } \\
\text { 县.jpg }\end{array}$ \\
\hline \multicolumn{2}{|l|}{ 27.菊科 Compositae } \\
\hline \multicolumn{2}{|l|}{ 1.顶羽菊属 Acroptilon } \\
\hline 1.顶羽菊 Acroptilon repens & http://www.nsii.org.cn/nsiiresources/media/photo/testPhoto/1d/original/顶羽菊（Acroptilon repens）段士民.jpg \\
\hline \multicolumn{2}{|l|}{ 2.牛蒡属 Arctium } \\
\hline 1.毛头牛蒡 Arctium tomentosum & $\begin{array}{l}\text { http://www.nsii.org.cn/nsiiresources/media/photo/testPhoto/1d/original/毛头牛蒡（Arctium tomentosum）段士民 } \\
\text { DSC_0119.jpg }\end{array}$ \\
\hline \multicolumn{2}{|l|}{ 3.矢车菊属 Centaurea } \\
\hline 1.针刺矢车菊 Centaurea iberica & http://www.nsii.org.cn/nsiiresources/media/photo/testPhoto/1d/original/针刺矢车菊 Centaurea iberica.jpg \\
\hline \multicolumn{2}{|l|}{ 4.菊苣属 Cichorium } \\
\hline 1.菊苣 Cichorium intybus & $\begin{array}{l}\text { http://www.nsii.org.cn/nsiiresources/media/photo/testPhoto/1d/original/菊苣（Cichorium intybus）段士民 } \\
\text { DSC_025.jpg }\end{array}$ \\
\hline \multicolumn{2}{|l|}{ 5.刺头菊属 Cousinia } \\
\hline 1.刺头菊 Cousinia affinis & $\begin{array}{l}\text { http://www.nsii.org.cn/nsiiresources/media/photo/testPhoto/1d/original/刺头菊（Cousinia affinis）段士民 } \\
\text { DSC_0009.jpg }\end{array}$ \\
\hline \multicolumn{2}{|l|}{ 6.蓝刺头属 Echinops } \\
\hline 1.大蓝刺头 Echinops talassicus & http://www.nsii.org.cn/nsiiresources/media/photo/testPhoto/1d/original/大蓝刺头（Echinops talassicus）段士民.jpg \\
\hline 2.天山蓝刺头 Echinops tjanschanicus & $\begin{array}{l}\text { http://www.nsii.org.cn/nsiiresources/media/photo/testPhoto/1d/original/天山蓝刺头（Echinops tjanschanicus），新 } \\
\text { 疆阿勒泰.jpg }\end{array}$ \\
\hline \multicolumn{2}{|l|}{ 7.苦苣菜属 Sonchus } \\
\hline 1.苦芭菜 Sonchus oleraceus & $\begin{array}{l}\text { http://www.nsii.org.cn/nsiiresources/media/photo/testPhoto/1d/original/苦芦菜（Sonchus oleraceus）段士民 } \\
\text { DSC_0047.jpg }\end{array}$ \\
\hline \multicolumn{2}{|l|}{ 8.异喙菊属 Heteracia } \\
\hline 1.异喙菊 Heteracia szovitsi & http://www.nsii.org.cn/nsiiresources/media/photo/testPhoto/1d/original/异喙菊（Heteracia szovitsii），新疆昌吉.jpg \\
\hline
\end{tabular}


许展慧, 刘诗尧, 赵芗, 涂文琴, 常诏峰, 张恩涛, 郭靖, 郑迪, 耿釷, 顾高营, 郭淳鹏, 郭璐璐, 王静, 徐春 阳, 彭钏, 杨腾, 崔梦琪, 孙伟成, 张剑坛, 刘皓天, 巴超群, 王鹤琪, 贾竞超, 武金洲, 肖翠, 马克平. 国内 8 款常用植物识别软件的识别能力评价. 生物多样性, 2020, 28 (4):524-533. http://www.biodiversityscience.net/CN/10.17520/biods.2019272

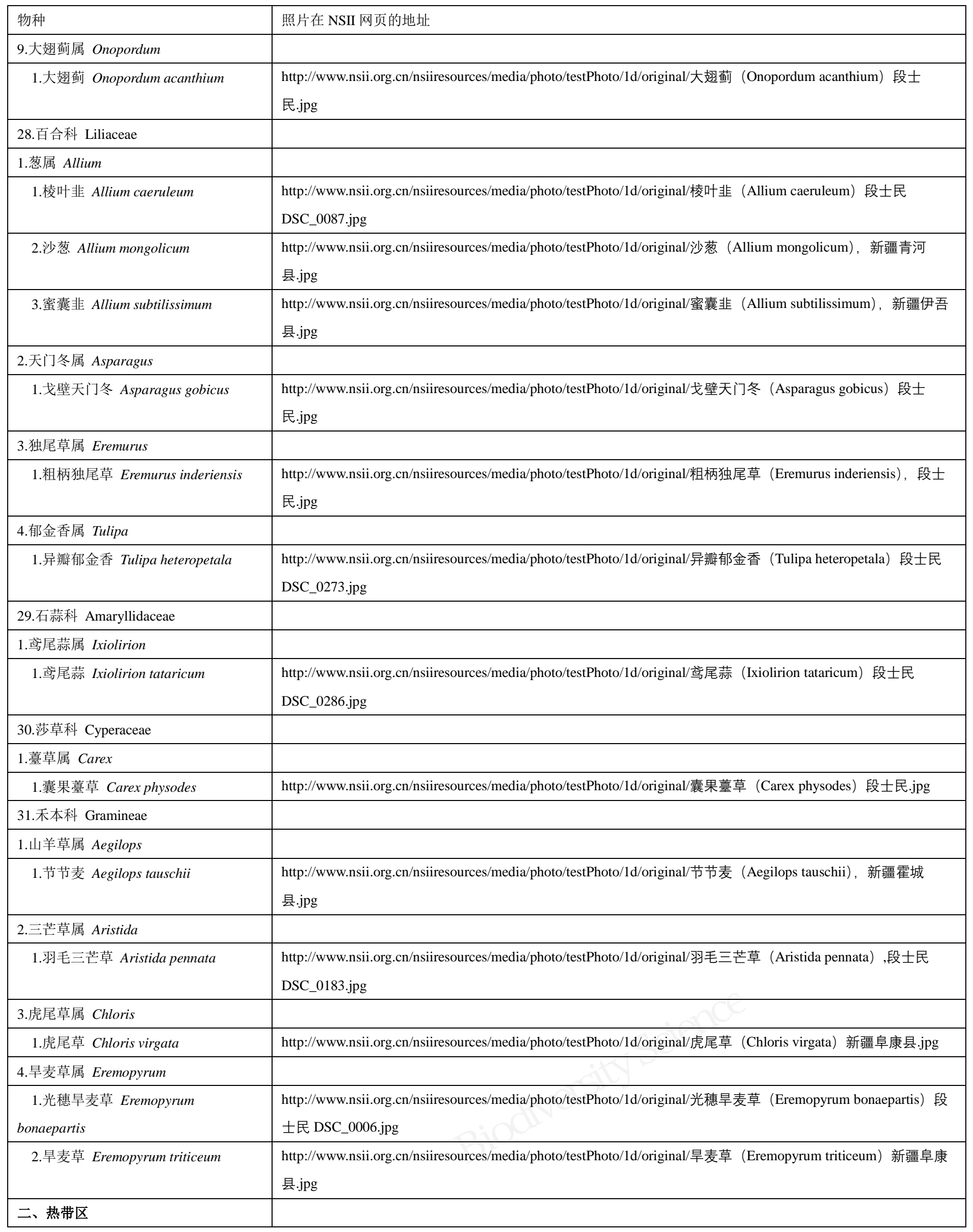


许展慧, 刘诗尧, 赵莹, 涂文琴, 常诏峰, 张恩涛, 郭靖, 郑迪, 耿均, 顾高营, 郭淳鹏, 郭璐璐, 王静, 徐春 阳, 彭钏, 杨腾, 崔梦琪, 孙伟成, 张剑坛, 刘皓天, 巴超群, 王鹤琪, 贾竞超, 武金洲, 肖翠, 马克平. 国内 8 款常用植物识别软件的识别能力评价. 生物多样性, 2020, 28 (4):524-533. http://www.biodiversityscience.net/CN/10.17520/biods.2019272

\begin{tabular}{|c|c|}
\hline 物种 & 照片在 NSII 网页的地址 \\
\hline \multicolumn{2}{|l|}{ 1.水䔊科 Parkeriaceae } \\
\hline \multicolumn{2}{|l|}{ 1.水偋属 Ceratopteris } \\
\hline 1.水硕 Ceratopteris thalictroides & $\begin{array}{l}\text { http://www.nsii.org.cn/nsiiresources/media/photo/testPhoto/4l/original/2 国家二级保护植物水蕨 Ceratopteris } \\
\text { thalictroides.JPG }\end{array}$ \\
\hline \multicolumn{2}{|l|}{ 2.水龙骨科 Polypodiaceae } \\
\hline \multicolumn{2}{|l|}{ 1.星烣属 Microsorum } \\
\hline 1.江南星硕 Microsorum fortunei & $\begin{array}{l}\text { http://www.nsii.org.cn/nsiiresources/media/photo/testPhoto/4l/original/江南星蕨 Microsorium fortunei } \\
\text { P1260590.JPG }\end{array}$ \\
\hline 2.江南星硕 Microsorum fortunei & http://www.nsii.org.cn/nsiiresources/media/photo/testPhoto/4l/original/江南星蕨 Microsorium fortunei.JPG \\
\hline \multicolumn{2}{|l|}{ 3.桑科 Moraceae } \\
\hline \multicolumn{2}{|l|}{ 1.号角树属 Cecropia } \\
\hline 1.号角树 Cecropia peltata & $\begin{array}{l}\text { http://www.nsii.org.cn/nsiiresources/media/photo/testPhoto/4l/original/号角树 Cecropia peltata 蚁栖树 } \\
\text { P2120297.JPG }\end{array}$ \\
\hline \multicolumn{2}{|l|}{ 2.榕属 Ficus } \\
\hline 1.高山榕 Ficus altissima & http://www.nsii.org.cn/nsiiresources/media/photo/testPhoto/4l/original/高榕 P1300652.JPG \\
\hline 2.垂叶榕 Ficus benjamina & http://www.nsii.org.cn/nsiiresources/media/photo/testPhoto/4l/original/垂叶榕 Ficus benjamina.JPG \\
\hline 3.水同木 Ficus fistulosa & http://www.nsii.org.cn/nsiiresources/media/photo/testPhoto/4l/original/水同木 Ficus fistulosa.JPG \\
\hline \multicolumn{2}{|l|}{ 3.牛筋滕属 Malaisia } \\
\hline 1.牛筋藤 Malaisia scandens & http://www.nsii.org.cn/nsiiresources/media/photo/testPhoto/4l/original/牛筋藤 Malaisia scandens P2040126.JPG \\
\hline \multicolumn{2}{|l|}{ 4.紫茉莉科 Nyctaginaceae } \\
\hline \multicolumn{2}{|l|}{ 1.黏腺果属 Commicarpus } \\
\hline $\begin{array}{l}\text { 1.中华黏腺果 Commicarpus } \\
\text { lantsangensis }\end{array}$ & $\begin{array}{l}\text { http://www.nsii.org.cn/nsiiresources/media/photo/testPhoto/4l/original/中华黏腺果 Commicarpus lantsangensis } \\
\text { P1290253.JPG }\end{array}$ \\
\hline $\begin{array}{l}\text { 2.中华黏腺果 Commicarpus } \\
\text { lantsangensis }\end{array}$ & $\begin{array}{l}\text { http://www.nsii.org.cn/nsiiresources/media/photo/testPhoto/4l/original/中华黏腺果 Commicarpus lantsangensis } \\
\text { P1290253.JPG }\end{array}$ \\
\hline \multicolumn{2}{|l|}{ 5.落葵科 Basellaceae } \\
\hline \multicolumn{2}{|l|}{ 1.落葵属 Basella } \\
\hline 1.落葵 Basella alba & http://www.nsii.org.cn/nsiiresources/media/photo/testPhoto/4l/original/落葵 Basella alba.JPG \\
\hline \multicolumn{2}{|l|}{ 6. 龙脑香科 Dipterocarpaceae } \\
\hline \multicolumn{2}{|l|}{ 1.坡垒属 Hopea } \\
\hline 1.坡垒 Hopea hainanensis & http://www.nsii.org.cn/nsiiresources/media/photo/testPhoto/4l/original/坡垒 Hopea hainanensis P1230517.JPG \\
\hline \multicolumn{2}{|l|}{ 7.藤黄科 Guttiferae } \\
\hline \multicolumn{2}{|l|}{ 1.红厚壳属 Calophyllum } \\
\hline 1.红厚壳 Calophyllum inophyllum & $\begin{array}{l}\text { http://www.nsii.org.cn/nsiiresources/media/photo/testPhoto/4l/original/8 红厚壳 Calophyllum inophyllum } \\
\text { P1210685.JPG }\end{array}$ \\
\hline \multicolumn{2}{|l|}{ 8.罂粟科 Papaveraceae } \\
\hline \multicolumn{2}{|l|}{ 1.蓟罂粟属 Argemone } \\
\hline 1.蓟罂粟 Argemone mexicana & http://www.nsii.org.cn/nsiiresources/media/photo/testPhoto/4l/original/蓟罂粟 Argemone mexicana P2041302.JPG \\
\hline \multicolumn{2}{|l|}{ 9.山柑科 Capparaceae } \\
\hline \multicolumn{2}{|l|}{ 1.白花菜属 CleomeCrassulaceae } \\
\hline 1.白花菜 Cleome gynandra & http://www.nsii.org.cn/nsiiresources/media/photo/testPhoto/4l/original/白花菜 Cleome gynandra.JPG \\
\hline 10.辣木科 Moringaceae & \\
\hline
\end{tabular}


许展慧, 刘诗尧, 赵芗, 涂文琴, 常诏峰, 张恩涛, 郭靖, 郑迪, 耿釷, 顾高营, 郭淳鹏, 郭璐璐, 王静, 徐春 阳, 彭钏, 杨腾, 崔梦琪, 孙伟成, 张剑坛, 刘皓天, 巴超群, 王鹤琪, 贾竞超, 武金洲, 肖翠, 马克平. 国内 8 款常用植物识别软件的识别能力评价. 生物多样性, 2020, 28 (4):524-533. http://www.biodiversityscience.net/CN/10.17520/biods.2019272

\begin{tabular}{|c|c|}
\hline 物种 & 照片在 NSII 网页的地址 \\
\hline \multicolumn{2}{|l|}{ 1.辣木属 Moringa } \\
\hline 1.象腿树 Moringa drouhardii & http://www.nsii.org.cn/nsiiresources/media/photo/testPhoto/4l/original/象腿树 Moringa drouhardii P1300518.JPG \\
\hline \multicolumn{2}{|l|}{ 11.豆科 Fabaceae } \\
\hline \multicolumn{2}{|l|}{ 1.合欢属 Albizia } \\
\hline 1.楹树 Albizia chinensis & http://www.nsii.org.cn/nsiiresources/media/photo/testPhoto/4l/original/15 楹树 Albizia chinensis P2130570.JPG \\
\hline \multicolumn{2}{|l|}{ 2.朱缨花属 Calliandra } \\
\hline 1.朱缨花 Calliandra haematocephala & http://www.nsii.org.cn/nsiiresources/media/photo/testPhoto/4l/original/朱缨花 DSC_5582.JPG \\
\hline \multicolumn{2}{|l|}{ 3.榼藤属 Entada } \\
\hline 1.榼藤 Entada phaseoloides & $\begin{array}{l}\text { http://www.nsii.org.cn/nsiiresources/media/photo/testPhoto/4l/original/榼藤 Entada phaseoloides 又叫眼睛豆 } \\
\text { P2060274.JPG }\end{array}$ \\
\hline \multicolumn{2}{|l|}{ 4.刺桐属 Erythrina } \\
\hline 1.鸡冠刺桐 Erythrina crista-galli & http://www.nsii.org.cn/nsiiresources/media/photo/testPhoto/4l/original/12 鸡冠刺桐 Erythrina crista-galli.JPG \\
\hline 2.乳黄刺桐 Erythrina mitis & http://www.nsii.org.cn/nsiiresources/media/photo/testPhoto/4l/original/20 乳黄刺桐 Erythrina mitis P2120403.JPG \\
\hline \multicolumn{2}{|l|}{ 5.木蓝属 Indigofera } \\
\hline 1.硬毛木蓝 Indigofera hirsuta & $\begin{array}{l}\text { http://www.nsii.org.cn/nsiiresources/media/photo/testPhoto/4l/original/29 硬毛木蓝. Indigofera hirsuta } \\
\text { P1290309.JPG }\end{array}$ \\
\hline 2.野青树 Indigofera suffruticosa & $\begin{array}{l}\text { http://www.nsii.org.cn/nsiiresources/media/photo/testPhoto/4l/original/27 野青树 Indigofera suffruticosa } \\
\text { P2040078.JPG }\end{array}$ \\
\hline \multicolumn{2}{|l|}{ 6.大翼豆属 Maackia } \\
\hline 1.大翼豆 Macroptilium atropurpureum & http://www.nsii.org.cn/nsiiresources/media/photo/testPhoto/4l/original/大翼豆 Macroptiliumatropurpureum.JPG \\
\hline \multicolumn{2}{|l|}{ 7.崖豆藤属 Millettia } \\
\hline 1.厚果崖豆藤 Millettia pachycarpa & http://www.nsii.org.cn/nsiiresources/media/photo/testPhoto/4l/original/厚果崖豆藤 Millettia pachycarpa.JPG \\
\hline \multicolumn{2}{|l|}{ 8.含羞草属 Mimosa } \\
\hline 1.巴西含羞草 Mimosa invisa & http://www.nsii.org.cn/nsiiresources/media/photo/testPhoto/4l/original/巴西含羞草 Mimosa invisa P1290112.JPG \\
\hline \multicolumn{2}{|l|}{ 9.无忧花属 Saraca } \\
\hline 1.无忧花 Saraca dives & http://www.nsii.org.cn/nsiiresources/media/photo/testPhoto/4l/original/14 无忧花 Saraca dives P2070202.JPG \\
\hline 2.无忧花 Saraca dives & http://www.nsii.org.cn/nsiiresources/media/photo/testPhoto/4l/original/14 无忧花 Saraca dives.JPG \\
\hline \multicolumn{2}{|l|}{ 12.酢浆草科 Oxalidaceae } \\
\hline \multicolumn{2}{|l|}{ 1.阳桃属 Averrhoa } \\
\hline 1.阳桃 Averrhoa carambola & http://www.nsii.org.cn/nsiiresources/media/photo/testPhoto/4l/original/阳桃 Averrhoa carambola P1260081.JPG \\
\hline \multicolumn{2}{|l|}{ 13.大戟科 Euphorbiaceae } \\
\hline \multicolumn{2}{|l|}{ 1.大戟属 Euphorbia } \\
\hline 1.霸王鞭 Euphorbia royleana & http://www.nsii.org.cn/nsiiresources/media/photo/testPhoto/4l/original/18 霸王鞭 Euphorbia royleana.JPG \\
\hline \multicolumn{2}{|l|}{ 2.海漆属 Excoecaria } \\
\hline $\begin{array}{l}\text { 1.红背桂花 Excoecaria } \\
\text { cochinchinensis }\end{array}$ & http://www.nsii.org.cn/nsiiresources/media/photo/testPhoto/4l/original/9 红背桂花 Excoecaria cochinchinensis.JPG \\
\hline $\begin{array}{l}\text { 2.红背桂花 Excoecaria } \\
\text { cochinchinensis }\end{array}$ & http://www.nsii.org.cn/nsiiresources/media/photo/testPhoto/4l/original/9 红背桂 Excoecaria cochinchinensis.JPG \\
\hline \multicolumn{2}{|l|}{ 3.橡胶树属 Hevea } \\
\hline 1.橡胶树 Hevea brasiliensis & http://www.nsii.org.cn/nsiiresources/media/photo/testPhoto/4l/original/橡胶树 Hevea brasiliensis P1260203.JPG \\
\hline 14.芸香科 Rutaceae & \\
\hline 1.小芸木属 Micromelum & \\
\hline
\end{tabular}


许展慧, 刘诗尧, 赵莹, 涂文琴, 常诏峰, 张恩涛, 郭靖, 郑迪, 耿均, 顾高营, 郭淳鹏, 郭璐璐, 王静, 徐春 阳, 彭钏, 杨腾, 崔梦琪, 孙伟成, 张剑坛, 刘皓天, 巴超群, 王鹤琪, 贾竞超, 武金洲, 肖翠, 马克平. 国内 8 款常用植物识别软件的识别能力评价. 生物多样性, 2020, 28 (4):524-533. http://www.biodiversityscience.net/CN/10.17520/biods.2019272

\begin{tabular}{|c|c|}
\hline 物种 & 照片在 NSII 网页的地址 \\
\hline 1.大管 Micromelum falcatum & http://www.nsii.org.cn/nsiiresources/media/photo/testPhoto/4l/original/26 大管 Micromelum falcatum.JPG \\
\hline \multicolumn{2}{|l|}{ 2.九里香属 Murraya } \\
\hline 1.九里香 Murraya exotica & http://www.nsii.org.cn/nsiiresources/media/photo/testPhoto/41/original/九里香 DSC_5256.JPG \\
\hline 2.九里香 Murraya exotica & http://www.nsii.org.cn/nsiiresources/media/photo/testPhoto/4l/original/九里香 Murraya exotica P2060013.JPG \\
\hline \multicolumn{2}{|l|}{ 15.漆树科 Anacardiaceae } \\
\hline \multicolumn{2}{|l|}{ 1.杧果属 Mangifera } \\
\hline 1.杧果 Mangifera indica & http://www.nsii.org.cn/nsiiresources/media/photo/testPhoto/4l/original/杧果 Mangifera indica P1310876.JPG \\
\hline 2.杧果 Mangifera indica & http://www.nsii.org.cn/nsiiresources/media/photo/testPhoto/4l/original/25 杧果 Mangifera indica 2.JPG \\
\hline 3.杧果 Mangifera indica & http://www.nsii.org.cn/nsiiresources/media/photo/testPhoto/4l/original/25 杧果 Mangifera indica 1.JPG \\
\hline \multicolumn{2}{|l|}{ 16.无患子科 Sapindaceae } \\
\hline \multicolumn{2}{|l|}{ 1.倒地铃属 Cardiospermum } \\
\hline 1.倒地铃 Cardiospermum halicacabum & $\begin{array}{l}\text { http://www.nsii.org.cn/nsiiresources/media/photo/testPhoto/4l/original/30 倒地铃 Candiospermum halicacabum } \\
\text { 2.JPG }\end{array}$ \\
\hline 2.倒地铃 Cardiospermum halicacabum & $\begin{array}{l}\text { http://www.nsii.org.cn/nsiiresources/media/photo/testPhoto/4l/original/30 倒地铃 Candiospermum halicacabum } \\
\text { 1.JPG }\end{array}$ \\
\hline \multicolumn{2}{|l|}{ 17.刺茉莉科 Salvadoraceae } \\
\hline \multicolumn{2}{|l|}{ 1.刺茉莉属 Azima } \\
\hline 1.刺茉莉 Azima sarmentosa & http://www.nsii.org.cn/nsiiresources/media/photo/testPhoto/4l/original/7 刺茉莉 Azima sarmentosa P2100602.JPG \\
\hline \multicolumn{2}{|l|}{ 18.红木科 Bixaceae } \\
\hline \multicolumn{2}{|l|}{ 1.红木属 Bixa } \\
\hline 1.红木 Bixa orellana & http://www.nsii.org.cn/nsiiresources/media/photo/testPhoto/4l/original/红木 Bixa orellana P1230761.JPG \\
\hline \multicolumn{2}{|l|}{ 19.桃金娘科 Myrtaceae } \\
\hline \multicolumn{2}{|l|}{ 1.蒲桃属 Syzygium } \\
\hline 1.洋蒲桃 Syzygium samarangense & $\begin{array}{l}\text { http://www.nsii.org.cn/nsiiresources/media/photo/testPhoto/4l/original/3 洋蒲桃 Syzygium samarangense } \\
\text { P1250760.JPG }\end{array}$ \\
\hline 2.洋蒲桃 Syzygium samarangense & $\begin{array}{l}\text { http://www.nsii.org.cn/nsiiresources/media/photo/testPhoto/4l/original/3 洋蒲桃 Syzygium samarangense } \\
\text { P2020308.JPG }\end{array}$ \\
\hline 3.洋蒲桃 Syzygium samarangense & http://www.nsii.org.cn/nsiiresources/media/photo/testPhoto/4l/original/洋蒲桃ＤSC_5207.JPG \\
\hline \multicolumn{2}{|l|}{ 20.野牡丹科 Melastomataceae } \\
\hline \multicolumn{2}{|l|}{ 1.野牡丹属 Melastoma } \\
\hline 1.毛菍 Melastoma sanguineum & $\begin{array}{l}\text { http://www.nsii.org.cn/nsiiresources/media/photo/testPhoto/4l/original/23 毛菍 Melastoma sanguineum } \\
\text { P1240838.JPG }\end{array}$ \\
\hline \multicolumn{2}{|l|}{ 21.锦葵科 Malvaceae } \\
\hline \multicolumn{2}{|l|}{ 1.木槿属 Hibiscus } \\
\hline 1.朱槿 Hibiscus rosa-sinensis & http://www.nsii.org.cn/nsiiresources/media/photo/testPhoto/4l/original/朱槿 DSC_5492.JPG \\
\hline 2.白背黄花稔 Sida rhombifolia & http://www.nsii.org.cn/nsiiresources/media/photo/testPhoto/4l/original/白背黄花稔 Sida rhombifolia.JPG \\
\hline \multicolumn{2}{|l|}{ 22.木棉科 Bombacaceae } \\
\hline \multicolumn{2}{|l|}{ 1.木棉属 Bombax } \\
\hline 1.木棉 Bombax ceiba & http://www.nsii.org.cn/nsiiresources/media/photo/testPhoto/4l/original/13 木棉Ｂombax ceiba.JPG \\
\hline \multicolumn{2}{|l|}{ 2.吉贝属 Ceiba } \\
\hline 1.吉贝 Ceiba pentandra & http://www.nsii.org.cn/nsiiresources/media/photo/testPhoto/4l/original/5 吉贝 Ceiba pentandra.JPG \\
\hline 2.吉贝 Ceiba pentandra & http://www.nsii.org.cn/nsiiresources/media/photo/testPhoto/4l/original/5 吉贝 Ceiba pentandra P1300696.JPG \\
\hline
\end{tabular}


许展慧, 刘诗尧, 赵芗, 涂文琴, 常诏峰, 张恩涛, 郭靖, 郑迪, 耿釷, 顾高营, 郭淳鹏, 郭璐璐, 王静, 徐春 阳, 彭钏, 杨腾, 崔梦琪, 孙伟成, 张剑坛, 刘皓天, 巴超群, 王鹤琪, 贾竞超, 武金洲, 肖翠, 马克平. 国内 8 款常用植物识别软件的识别能力评价. 生物多样性, 2020, 28 (4):524-533. http://www.biodiversityscience.net/CN/10.17520/biods.2019272

\begin{tabular}{|c|c|}
\hline 物种 & 照片在 NSII 网页的地址 \\
\hline 3.吉贝 Ceiba pentandra & http://www.nsii.org.cn/nsiiresources/media/photo/testPhoto/4l/original/吉贝 Ceiba pentandraＰ1300696.JPG \\
\hline \multicolumn{2}{|l|}{ 23.梧桐科 Sterculiaceae } \\
\hline \multicolumn{2}{|l|}{ 1.可乐果属 Cola } \\
\hline 1.光亮可乐果 Cola nitida & http://www.nsii.org.cn/nsiiresources/media/photo/testPhoto/4l/original/4 光亮可乐果 Cola nitida.JPG \\
\hline \multicolumn{2}{|l|}{ 2.马松子属 Melochia } \\
\hline 1.马松子 Melochia corchorifolia & http://www.nsii.org.cn/nsiiresources/media/photo/testPhoto/4l/original/马松子 Melochia corchorifolia.JPG \\
\hline \multicolumn{2}{|l|}{ 3.苹婆属 Sterculia } \\
\hline 1.假苹婆 Sterculia lanceolata & http://www.nsii.org.cn/nsiiresources/media/photo/testPhoto/4l/original/假苹婆 Sterculia lanceolataＰ2050245.JPG \\
\hline \multicolumn{2}{|l|}{ 24.紫金牛科 Myrsinaceae } \\
\hline \multicolumn{2}{|l|}{ 1.紫金牛属 Ardisia } \\
\hline 1.雪下红 Ardisia villosa & http://www.nsii.org.cn/nsiiresources/media/photo/testPhoto/4l/original/雪下红 Ardisia villosa P2050129.JPG \\
\hline \multicolumn{2}{|l|}{ 25.杜鹃花科 Ericaceae } \\
\hline \multicolumn{2}{|l|}{ 1.杜鹃属 Rhododendron } \\
\hline 1.多花杜鹃 Rhododendron cavaleriei & $\begin{array}{l}\text { http://www.nsii.org.cn/nsiiresources/media/photo/testPhoto/4l/original/6 多花杜鹃 Rhododendron cavaleriei } \\
\text { P1240776.JPG }\end{array}$ \\
\hline \multicolumn{2}{|l|}{ 26.白花丹科 Plumbaginaceae } \\
\hline \multicolumn{2}{|l|}{ 1.白花丹属 Plumbago } \\
\hline 1.白花丹 Plumbago zeylanica & http://www.nsii.org.cn/nsiiresources/media/photo/testPhoto/4l/original/白花丹 Plumbago zeylanica.JPG \\
\hline \multicolumn{2}{|l|}{ 27.山榄科 Sapotaceae } \\
\hline \multicolumn{2}{|l|}{ 1.铁线子属 Manilkara } \\
\hline 1.人心果 Manilkara zapota & http://www.nsii.org.cn/nsiiresources/media/photo/testPhoto/4l/original/人心果 Manilkara zapota.JPG \\
\hline \multicolumn{2}{|l|}{ 27.夹竹桃科 Apocynaceae } \\
\hline \multicolumn{2}{|l|}{ 1.黄蝉属 Allamanda } \\
\hline 1.紫蝉花 Allamanda violacea & $\begin{array}{l}\text { http://www.nsii.org.cn/nsiiresources/media/photo/testPhoto/4l/original/16 紫蝉花 Allamanda violacea } \\
\text { P2020939.JPG }\end{array}$ \\
\hline 2.软枝黄蝉 Allemanda cathartica & http://www.nsii.org.cn/nsiiresources/media/photo/testPhoto/4l/original/21 软枝黄蝉 Allemanda cathartica.JPG \\
\hline \multicolumn{2}{|l|}{ 2.鸡骨常山属 Alstonia } \\
\hline 1.糖胶树 Allamanda violacea & http://www.nsii.org.cn/nsiiresources/media/photo/testPhoto/4l/original/糖胶树 Alstonia scholaris P1300723.JPG \\
\hline \multicolumn{2}{|l|}{ 3.夢芙木属 Rauvolfia } \\
\hline 1.萝芙木 Rauvolfia verticillata & http://www.nsii.org.cn/nsiiresources/media/photo/testPhoto/4l/original/萝芙木 Rauvolfia verticillata P1240492.JPG \\
\hline \multicolumn{2}{|l|}{ 28.夢藦科 Asclepiadaceae } \\
\hline \multicolumn{2}{|l|}{ 1.牛角瓜属 Calotropis } \\
\hline 1.牛角瓜 Calotropis gigantea & http://www.nsii.org.cn/nsiiresources/media/photo/testPhoto/4l/original/牛角瓜 Calotropis gigantea.JPG \\
\hline \multicolumn{2}{|l|}{ 29.茜草科 Rubiaceae } \\
\hline \multicolumn{2}{|l|}{ 1.咖啡属 Coffea } \\
\hline 1.大粒咖啡 Coffea liberica & http://www.nsii.org.cn/nsiiresources/media/photo/testPhoto/4l/original/17 大粒咖啡 Coffea liberica.JPG \\
\hline \multicolumn{2}{|l|}{ 2.九节属 Psychotria } \\
\hline 1.九节 Psychotria rubra & http://www.nsii.org.cn/nsiiresources/media/photo/testPhoto/4l/original/24 九节 Psychotria rubra.JPG \\
\hline \multicolumn{2}{|l|}{ 3.墨苜宿属 Richardia } \\
\hline 1.墨苜蕧 Richardia brasiliensis & http://www.nsii.org.cn/nsiiresources/media/photo/testPhoto/4l/original/墨苜宿 Richardia brasiliensis.JPG \\
\hline 2.墨苜宿 Richardia brasiliensis & http://www.nsii.org.cn/nsiiresources/media/photo/testPhoto/4l/original/墨苜蓿 Richardia brasiliensis (2).JPG \\
\hline
\end{tabular}


许展慧, 刘诗尧, 赵芗, 涂文琴, 常诏峰, 张恩涛, 郭靖, 郑迪, 耿釷, 顾高营, 郭淳鹏, 郭璐璐, 王静, 徐春 阳, 彭钏, 杨腾, 崔梦琪, 孙伟成, 张剑坛, 刘皓天, 巴超群, 王鹤琪, 贾竞超, 武金洲, 肖翠, 马克平. 国内 8 款常用植物识别软件的识别能力评价. 生物多样性, 2020, 28 (4):524-533. http://www.biodiversityscience.net/CN/10.17520/biods.2019272

\begin{tabular}{|c|c|}
\hline 物种 & 照片在 NSII 网页的地址 \\
\hline \multicolumn{2}{|l|}{ 30.旋花科 Convolvulaceae } \\
\hline \multicolumn{2}{|l|}{ 1.番薯属 Ipomoea } \\
\hline 1.䔨菜 Ipomoea aquatica & http://www.nsii.org.cn/nsiiresources/media/photo/testPhoto/4l/original/䔨菜 Ipomoea aquatica.JPG \\
\hline \multicolumn{2}{|l|}{ 2.金鱼花属 Mina } \\
\hline 1.金鱼花 Mina lobata & http://www.nsii.org.cn/nsiiresources/media/photo/testPhoto/4l/original/金鱼花 DSC_5667.JPG \\
\hline \multicolumn{2}{|l|}{ 31.马鞭草科 Verbenaceae } \\
\hline \multicolumn{2}{|l|}{ 1.大青属 Clerodendrum } \\
\hline $\begin{array}{l}\text { 1.烟火树 Clerodendrum } \\
\text { quadriloculare }\end{array}$ & $\begin{array}{l}\text { http://www.nsii.org.cn/nsiiresources/media/photo/testPhoto/4l/original/28 烟火树 Clerodendrum quadriloculare } \\
\text { P2031092.JPG }\end{array}$ \\
\hline \multicolumn{2}{|l|}{ 2.假连翘属 Duranta } \\
\hline 1.假连尧 Duranta erecta & http://www.nsii.org.cn/nsiiresources/media/photo/testPhoto/4l/original/假连翘 Duranta erecta P2050293.JPG \\
\hline \multicolumn{2}{|l|}{ 32.唇形科 Labiatae } \\
\hline \multicolumn{2}{|l|}{ 1.四棱草属 Stachytarpheta } \\
\hline $\begin{array}{l}\text { 1.假马鞭草 Stachytarpheta } \\
\text { jamaicensis }\end{array}$ & http://www.nsii.org.cn/nsiiresources/media/photo/testPhoto/4l/original/假马鞭草 Stachytarpheta jamaicensis.JPG \\
\hline \multicolumn{2}{|l|}{ 33.紫葳科 Bignoniaceae } \\
\hline \multicolumn{2}{|l|}{ 1.葫芦树属 Crescentia } \\
\hline 1.葫芦树 Crescentia cujete & $\begin{array}{l}\text { http://www.nsii.org.cn/nsiiresources/media/photo/testPhoto/4l/original/葫芦树 Crescentia cujete 铁西瓜 } \\
\text { P1250335.JPG }\end{array}$ \\
\hline \multicolumn{2}{|l|}{ 2.蜡烛树属 Parmentiera } \\
\hline 1.蜡烛树 Parmentiera cerifera & http://www.nsii.org.cn/nsiiresources/media/photo/testPhoto/4l/original/蜡烛树 Parmentiera cereifera P2070278.JPG \\
\hline \multicolumn{2}{|l|}{ 3.炮仗藤属 Pyrostegia } \\
\hline 1.炮仗花 Pyrostegia venusta & http://www.nsii.org.cn/nsiiresources/media/photo/testPhoto/4l/original/19 炮仗花 Pyrostegia venusta.JPG \\
\hline \multicolumn{2}{|l|}{ 4.火焰树属 Spathodea } \\
\hline 1.火焰树 Spathodea campanulata & http://www.nsii.org.cn/nsiiresources/media/photo/testPhoto/4l/original/11 火焰树 Spathodea campanulata.JPG \\
\hline \multicolumn{2}{|l|}{ 5.风铃木属 Tabebuia } \\
\hline 1.粉花风铃木 Tabebuia chrysantha & http://www.nsii.org.cn/nsiiresources/media/photo/testPhoto/4l/original/10 粉花风铃木 Tabebuia chrysantha.JPG \\
\hline \multicolumn{2}{|l|}{ 34.爵床科 Acanthaceae } \\
\hline \multicolumn{2}{|l|}{ 1.十万错属 Asystasia } \\
\hline 1.小花宽叶十万错 Asystasia gangetica & $\begin{array}{l}\text { http://www.nsii.org.cn/nsiiresources/media/photo/testPhoto/4l/original/小花宽叶十万错 Asystasia } \\
\text { gangeticaP1290071.JPG }\end{array}$ \\
\hline \multicolumn{2}{|l|}{ 2.狗肝菜属 Dicliptera } \\
\hline 1.印度狗肝菜 Dicliptera bupleuroides & $\begin{array}{l}\text { http://www.nsii.org.cn/nsiiresources/media/photo/testPhoto/4l/original/印度狗肝菜 Dicliptera bupleuroides } \\
\text { P2050098.JPG }\end{array}$ \\
\hline \multicolumn{2}{|l|}{ 3.叉花草属 Diflugossa } \\
\hline 1.叉花草 Diflugossa colorata & http://www.nsii.org.cn/nsiiresources/media/photo/testPhoto/4l/original/叉花草 Diflugossa colorata.JPG \\
\hline \multicolumn{2}{|l|}{ 4.喜花草属 Eranthemum } \\
\hline 1.喜花草 Eranthemum pulchellum & $\begin{array}{l}\text { http://www.nsii.org.cn/nsiiresources/media/photo/testPhoto/4l/original/喜花草 Eranthemum pulchellum P2020967 } \\
\text { 6.JPG }\end{array}$ \\
\hline 2.喜花草 Eranthemum pulchellum & http://www.nsii.org.cn/nsiiresources/media/photo/testPhoto/4l/original/喜花草 DSC_5776.JPG \\
\hline \multicolumn{2}{|l|}{ 5.山牵牛属 Thunbergia } \\
\hline 1.桂叶山牵牛 Thunbergia laurifolia & http://www.nsii.org.cn/nsiiresources/media/photo/testPhoto/4l/original/桂叶山牵牛 Thunbergia laurifolia.JPG \\
\hline
\end{tabular}


许展慧, 刘诗尧, 赵芗, 涂文琴, 常诏峰, 张恩涛, 郭靖, 郑迪, 耿釷, 顾高营, 郭淳鹏, 郭璐璐, 王静, 徐春 阳, 彭钏, 杨腾, 崔梦琪, 孙伟成, 张剑坛, 刘皓天, 巴超群, 王鹤琪, 贾竞超, 武金洲, 肖翠, 马克平. 国内 8 款常用植物识别软件的识别能力评价. 生物多样性, 2020, 28 (4):524-533. http://www.biodiversityscience.net/CN/10.17520/biods.2019272

\begin{tabular}{|c|c|}
\hline 物种 & 照片在 NSII 网页的地址 \\
\hline \multicolumn{2}{|l|}{ 35.草海桐科 Goodeniaceae } \\
\hline \multicolumn{2}{|l|}{ 1.草海桐属 Scaevola } \\
\hline 1.草海桐 Scaevola sericea & http://www.nsii.org.cn/nsiiresources/media/photo/testPhoto/4l/original/草海桐 Scaevola sericea.JPG \\
\hline \multicolumn{2}{|l|}{ 36.菊科 Compositae } \\
\hline \multicolumn{2}{|l|}{ 1.菊芹属 Erechtites } \\
\hline $\begin{array}{l}\text { 1.败酱叶菊芹 Erechtites } \\
\text { valerianaefolia }\end{array}$ & $\begin{array}{l}\text { http://www.nsii.org.cn/nsiiresources/media/photo/testPhoto/4l/original/败酱叶菊芹 Erechtites valerianaefolia } \\
\text { P2041476.JPG }\end{array}$ \\
\hline \multicolumn{2}{|l|}{ 2.蟛蜞菊属 Wedelia } \\
\hline 1.三裂螘蜞菊 Wedelia trilobata & http://www.nsii.org.cn/nsiiresources/media/photo/testPhoto/4l/original/三裂蟛蜞菊 Wedelia trilobata P1300592.JPG \\
\hline \multicolumn{2}{|l|}{ 3.孪花菊属 Wollastonia } \\
\hline $\begin{array}{l}\text { 1.孪花菊 Wollastonia } \\
\text { bifloraWollastonia biflora }\end{array}$ & http://www.nsii.org.cn/nsiiresources/media/photo/testPhoto/4l/original/孪花菊 Melanthera biflora.JPG \\
\hline \multicolumn{2}{|l|}{ 37.水鳖科 Hydrocharitaceae } \\
\hline \multicolumn{2}{|l|}{ 1.水车前属 Ottelia } \\
\hline 1.水菜花 Ottelia cordata & $\begin{array}{l}\text { http://www.nsii.org.cn/nsiiresources/media/photo/testPhoto/4l/original/1 国家二级保护植物水菜花 Ottelia cordata } \\
\text { P1311321 (1).JPG }\end{array}$ \\
\hline 2.水菜花 Ottelia cordata & http://www.nsii.org.cn/nsiiresources/media/photo/testPhoto/4l/original/1 水菜花 Ottelia cordata P1311321 (2).JPG \\
\hline \multicolumn{2}{|l|}{ 37.鸭跖草科 Commelinaceae } \\
\hline \multicolumn{2}{|l|}{ 1.吊竹梅属 Tradescantia } \\
\hline 1.吊竹梅 Tradescantia zebrina & http://www.nsii.org.cn/nsiiresources/media/photo/testPhoto/4l/original/吊竹梅 DSC_5376.JPG \\
\hline 2.吊竹梅 Tradescantia zebrina & http://www.nsii.org.cn/nsiiresources/media/photo/testPhoto/4l/original/吊竹梅 Tradescantia zebrina.JPG \\
\hline \multicolumn{2}{|l|}{ 38.棕㭣科 Palmae } \\
\hline \multicolumn{2}{|l|}{ 1.霸王棕属 Bismarckia } \\
\hline 1.霸王棕 Bismarckia nobilis & http://www.nsii.org.cn/nsiiresources/media/photo/testPhoto/4l/original/霸王棕 P2010147.JPG \\
\hline \multicolumn{2}{|l|}{ 2.蒲葵属 Livistona } \\
\hline 1.蒲葵 Livistona chinensis & http://www.nsii.org.cn/nsiiresources/media/photo/testPhoto/4l/original/蒲葵 Livistona chinensis P1310988.JPG \\
\hline \multicolumn{2}{|l|}{ 3.水椰属 Nypa } \\
\hline 1.水椰 Nypa fructicans & http://www.nsii.org.cn/nsiiresources/media/photo/testPhoto/4l/original/水椰 Nypa fructicans P2041652.JPG \\
\hline \multicolumn{2}{|l|}{ 4.王棕属 Roystonea } \\
\hline 1.王棕 Roystonea regia & http://www.nsii.org.cn/nsiiresources/media/photo/testPhoto/4l/original/22 王棕 Roystonea regia.JPG \\
\hline \multicolumn{2}{|l|}{ 5.狐尾椰属 Wodyetia } \\
\hline 1.狐尾椰 Wodyetia bifurcata & http://www.nsii.org.cn/nsiiresources/media/photo/testPhoto/4l/original/狐尾椰 Wodyetia bifurcata.JPG \\
\hline \multicolumn{2}{|l|}{ 39.露兜树科 Pandanaceae } \\
\hline \multicolumn{2}{|l|}{ 1.露淣树属 Pandanus } \\
\hline 1.红刺露淣树 Pandanus utilis & http://www.nsii.org.cn/nsiiresources/media/photo/testPhoto/4l/original/红刺露兒树 Pandanus utilis P2010027.JPG \\
\hline 2.红刺露兜树 Pandanus utilis & http://www.nsii.org.cn/nsiiresources/media/photo/testPhoto/4l/original/红刺露㿟树 P2010027.JPG \\
\hline 三、亚热带区 & \\
\hline 1.里白科 Gleicheniaceae & \\
\hline 1.里白属 Hicriopteris & \\
\hline 1.里白 Diplopterygium glaucum & $\begin{array}{l}\text { http://www.nsii.org.cn/nsiiresources/media/photo/testPhoto/3c/original/Diplopterygium glaucum_里白_浙江古田山_ } \\
\text { 陈涁 2008-05-14 17-03-46.jpg }\end{array}$ \\
\hline
\end{tabular}


许展慧, 刘诗尧, 赵莹, 涂文琴, 常诏峰, 张恩涛, 郭靖, 郑迪, 耿均, 顾高营, 郭淳鹏, 郭璐璐, 王静, 徐春 阳, 彭钏, 杨腾, 崔梦琪, 孙伟成, 张剑坛, 刘皓天, 巴超群, 王鹤琪, 贾竞超, 武金洲, 肖翠, 马克平. 国内 8 款常用植物识别软件的识别能力评价. 生物多样性, 2020, 28 (4):524-533. http://www.biodiversityscience.net/CN/10.17520/biods.2019272

\begin{tabular}{|c|c|}
\hline 物种 & 照片在 NSII 网页的地址 \\
\hline 2.里白 Diplopterygium glaucum & $\begin{array}{l}\text { http://www.nsii.org.cn/nsiiresources/media/photo/testPhoto/3c/original/Diplopterygium glaucum_里白_浙江古田山_ } \\
\text { 陈涁 2008-05-14 17-03-36.jpg }\end{array}$ \\
\hline \multicolumn{2}{|l|}{ 2.芒萁属 Dicranopteris } \\
\hline 1.芒萁 Dicranopteris pedata & $\begin{array}{l}\text { http://www.nsii.org.cn/nsiiresources/media/photo/testPhoto/3c/original/Dicranopteris pedata_芒萁_浙江古田山_陈 } \\
\text { 涁 2008-05-14 17-03-21.jpg }\end{array}$ \\
\hline \multicolumn{2}{|l|}{ 2.鳞毛蕨科 Dryopteridaceae } \\
\hline \multicolumn{2}{|l|}{ 1.复叶耳蕨属 Arachniodes } \\
\hline 1.斜方复叶耳烣 Arachniodes amabilis & $\begin{array}{l}\text { http://www.nsii.org.cn/nsiiresources/media/photo/testPhoto/3c/original/Arachniodes amabilis_斜方复叶耳蕨_浙江古 } \\
\text { 田山_陈彬 2008-05-12 17-04-44.jpg }\end{array}$ \\
\hline 2.刺头复叶耳蕨 Arachniodes aristata & $\begin{array}{l}\text { http://www.nsii.org.cn/nsiiresources/media/photo/testPhoto/3c/original/Arachniodes aristata_刺头复叶耳蕨_浙江古 } \\
\text { 田山_陈涁 2008-05-15 17-41-55.jpg }\end{array}$ \\
\hline 3.刺头复叶耳蕨 Arachniodes aristata & $\begin{array}{l}\text { http://www.nsii.org.cn/nsiiresources/media/photo/testPhoto/3c/original/Arachniodes aristata_刺头复叶耳蕨_浙江古 } \\
\text { 田山_陈涁 2008-05-16 10-25-02.jpg }\end{array}$ \\
\hline \multicolumn{2}{|l|}{ 2.贯众属 Cyrtomium } \\
\hline 1.镰羽贯众 Cyrtomium balansae & $\begin{array}{l}\text { http://www.nsii.org.cn/nsiiresources/media/photo/testPhoto/3c/original/Cyrtomium balansae_镰羽贯众_浙江古田山 } \\
\text { _陈涁 2008-05-16 10-06-56.jpg }\end{array}$ \\
\hline 2.镰羽贯众 Cyrtomium balansae & $\begin{array}{l}\text { http://www.nsii.org.cn/nsiiresources/media/photo/testPhoto/3c/original/Cyrtomium balansae_镰羽贯众_浙江古田山 } \\
\text { _陈涁 2008-05-16 10-06-21.jpg }\end{array}$ \\
\hline 3.镰羽贯众 Cyrtomium balansae & $\begin{array}{l}\text { http://www.nsii.org.cn/nsiiresources/media/photo/testPhoto/3c/original/Cyrtomium balansae_镰羽贯众_浙江古田山 } \\
\text { _陈涁 2008-05-16 10-06-02.jpg }\end{array}$ \\
\hline 4.镰羽贯众 Cyrtomium balansae & $\begin{array}{l}\text { http://www.nsii.org.cn/nsiiresources/media/photo/testPhoto/3c/original/Cyrtomium balansae_镰羽贯众_浙江古田山 } \\
\text { _陈涁 2008-05-16 10-05-30.jpg }\end{array}$ \\
\hline \multicolumn{2}{|l|}{ 3.舌蕨科 Elaphoglossaceae } \\
\hline \multicolumn{2}{|l|}{ 1.舌蕨属 Elaphoglossum } \\
\hline 1.华南舌萍 Elaphoglossum yoshinagae & $\begin{array}{l}\text { http://www.nsii.org.cn/nsiiresources/media/photo/testPhoto/3c/original/Elaphoglossum yoshinagae_华南舌蕨_浙江 } \\
\text { 古田山_陈涁 2008-05-13 15-37-38.jpg }\end{array}$ \\
\hline 2.华南舌硕 Elaphoglossum yoshinagae & $\begin{array}{l}\text { http://www.nsii.org.cn/nsiiresources/media/photo/testPhoto/3c/original/Elaphoglossum yoshinagae_华南舌蕨_浙江 } \\
\text { 古田山_陈涁 2008-05-13 15-37-23.jpg }\end{array}$ \\
\hline \multicolumn{2}{|l|}{ 4.书带硕科 Vittariaceae } \\
\hline \multicolumn{2}{|l|}{ 1.书带鄀属 Haplopteris } \\
\hline 1.书带硕 Haplopteris flexuosa & $\begin{array}{l}\text { http://www.nsii.org.cn/nsiiresources/media/photo/testPhoto/3c/original/Haplopteris flexuosa_书带蕨_浙江古田山_ } \\
\text { 陈涁 2008-05-16 09-46-49.jpg }\end{array}$ \\
\hline \multicolumn{2}{|l|}{ 5.银杏科 Ginkgoaceae } \\
\hline \multicolumn{2}{|l|}{ 1.银杏属 Ginkgo } \\
\hline 1.银杏 Ginkgo biloba & $\begin{array}{l}\text { http://www.nsii.org.cn/nsiiresources/media/photo/testPhoto/3c/original/Ginkgo biloba_银杏_浙江古田山_陈彬 } \\
\text { 2008-05-15 18-26-18.jpg }\end{array}$ \\
\hline \multicolumn{2}{|l|}{ 6.桦木科 Betulaceae } \\
\hline \multicolumn{2}{|l|}{ 1.鹅耳枥属 Carpinus } \\
\hline 1.鹅耳枥 Carpinus turczaninowii & $\begin{array}{l}\text { http://www.nsii.org.cn/nsiiresources/media/photo/testPhoto/3c/original/Carpinus turczaninowii_我耳枥_浙江古田山 } \\
\text { _陈涁 2008-05-14 09-12-42.jpg }\end{array}$ \\
\hline \multicolumn{2}{|l|}{ 7.壳斗科 Fagaceae } \\
\hline 1.栗属 Castanea & \\
\hline
\end{tabular}


许展慧, 刘诗尧, 赵莹, 涂文琴, 常诏峰, 张恩涛, 郭靖, 郑迪, 耿均, 顾高营, 郭淳鹏, 郭璐璐, 王静, 徐春 阳, 彭钏, 杨腾, 崔梦琪, 孙伟成, 张剑坛, 刘皓天, 巴超群, 王鹤琪, 贾竞超, 武金洲, 肖翠, 马克平. 国内 8 款常用植物识别软件的识别能力评价. 生物多样性, 2020, 28 (4):524-533. http://www.biodiversityscience.net/CN/10.17520/biods.2019272

\begin{tabular}{|c|c|}
\hline 物种 & 照片在 NSII 网页的地址 \\
\hline 1.栗 Castanea mollissima & $\begin{array}{l}\text { http://www.nsii.org.cn/nsiiresources/media/photo/testPhoto/3c/original/Castanea mollissima_栗_浙江古田山_陈涁 } \\
\text { 2008-05-12 18-19-15.jpg }\end{array}$ \\
\hline 2.栗 Castanea mollissima & $\begin{array}{l}\text { http://www.nsii.org.cn/nsiiresources/media/photo/testPhoto/3c/original/Castanea mollissima_栗_浙江古田山_陈涁 } \\
\text { 2008-05-14 07-59-35.jpg }\end{array}$ \\
\hline 3.茅栗 Castanea seguinii & $\begin{array}{l}\text { http://www.nsii.org.cn/nsiiresources/media/photo/testPhoto/3c/original/Castanea seguinii_茅栗_浙江古田山_陈涁 } \\
\text { 2008-05-14 16-44-50.jpg }\end{array}$ \\
\hline \multicolumn{2}{|l|}{ 2.青冈属 Cyclobalanopsis } \\
\hline 1.青冈 Cyclobalanopsis glauca & $\begin{array}{l}\text { http://www.nsii.org.cn/nsiiresources/media/photo/testPhoto/3c/original/Cyclobalanopsis glauca_青冈_浙江古田山_ } \\
\text { 陈涁 2008-05-15 15-55-16.jpg }\end{array}$ \\
\hline 2.青冈 Cyclobalanopsis glauca & $\begin{array}{l}\text { http://www.nsii.org.cn/nsiiresources/media/photo/testPhoto/3c/original/Cyclobalanopsis glauca_青冈_浙江古田山_ } \\
\text { 陈涁 2008-05-15 15-55-40.jpg }\end{array}$ \\
\hline \multicolumn{2}{|l|}{ 3.水青冈属 Cyclobalanopsis } \\
\hline 1.水青冈 Fagus longipetiolata & $\begin{array}{l}\text { http://www.nsii.org.cn/nsiiresources/media/photo/testPhoto/3c/original/Fagus longipetiolata_水青冈_浙江古田山_陈 } \\
\text { 涁 2008-05-14 08-08-54.jpg }\end{array}$ \\
\hline \multicolumn{2}{|l|}{ 8.桑科 Moraceae } \\
\hline \multicolumn{2}{|l|}{ 1.榕属 Ficus } \\
\hline 1.天仙果 Ficus erecta & $\begin{array}{l}\text { http://www.nsii.org.cn/nsiiresources/media/photo/testPhoto/3c/original/Ficus erecta_天仙果_浙江古田山_陈涁 } \\
\text { 2008-05-16 09-58-21.jpg }\end{array}$ \\
\hline 2.天仙果 Ficus erecta & $\begin{array}{l}\text { http://www.nsii.org.cn/nsiiresources/media/photo/testPhoto/3c/original/Ficus erecta_天仙果_浙江古田山_陈涁 } \\
\text { 2008-05-16 09-58-41.jpg }\end{array}$ \\
\hline \multicolumn{2}{|l|}{ 9.菖麻科 Urticaceae } \\
\hline \multicolumn{2}{|l|}{ 1.糯米团属 Gonostegia } \\
\hline 1.糯米团 Gonostegia hirta & $\begin{array}{l}\text { http://www.nsii.org.cn/nsiiresources/media/photo/testPhoto/3c/original/Gonostegia hirta_糯米团_浙江古田山_陈涁 } \\
\text { 2008-05-13 17-08-37.jpg }\end{array}$ \\
\hline \multicolumn{2}{|l|}{ 10.蜡梅科 Calycanthaceae } \\
\hline \multicolumn{2}{|l|}{ 1.蜡梅属 Chimonanthus } \\
\hline 1.柳叶蜡梅 Chimonanthus salicifolius & $\begin{array}{l}\text { http://www.nsii.org.cn/nsiiresources/media/photo/testPhoto/3c/original/Chimonanthus salicifolius_柳叶蜡梅_浙江古 } \\
\text { 田山_陈涁 2008-05-15 14-58-14.jpg }\end{array}$ \\
\hline \multicolumn{2}{|l|}{ 11.小檗科 Berberidaceae } \\
\hline \multicolumn{2}{|l|}{ 1.鬼臼属 Dysosma } \\
\hline 1.八角莲 Dysosma versipellis & $\begin{array}{l}\text { http://www.nsii.org.cn/nsiiresources/media/photo/testPhoto/3c/original/Dysosma versipellis_八角莲_浙江古田山_陈 } \\
\text { 涁 2008-05-14 17-40-56.jpg }\end{array}$ \\
\hline 2.八角莲 Dysosma versipellis & $\begin{array}{l}\text { http://www.nsii.org.cn/nsiiresources/media/photo/testPhoto/3c/original/Dysosma versipellis_八角莲_浙江古田山_陈 } \\
\text { 涁 2008-05-14 17-40-58.jpg }\end{array}$ \\
\hline \multicolumn{2}{|l|}{ 12.防己科 Menispermaceae } \\
\hline \multicolumn{2}{|l|}{ 1.木防己属 Cocculus } \\
\hline 1.木防己 Cocculus orbiculatus & $\begin{array}{l}\text { http://www.nsii.org.cn/nsiiresources/media/photo/testPhoto/3c/original/Cocculus orbiculatus_木防已_浙江古田山_ } \\
\text { 陈涁 2008-05-13 10-47-37.jpg }\end{array}$ \\
\hline \multicolumn{2}{|l|}{ 13.三白草科 Saururaceae } \\
\hline \multicolumn{2}{|l|}{ 1.哉菜属 Houttuynia } \\
\hline 1.藏菜 Houttuynia cordata & $\begin{array}{l}\text { http://www.nsii.org.cn/nsiiresources/media/photo/testPhoto/3c/original/Houttuynia cordata_载菜_浙江古田山_陈涁 } \\
\text { 2008-05-12 17-56-25.jpg }\end{array}$ \\
\hline
\end{tabular}


许展慧, 刘诗尧, 赵芗, 涂文琴, 常诏峰, 张恩涛, 郭靖, 郑迪, 耿釷, 顾高营, 郭淳鹏, 郭璐璐, 王静, 徐春 阳, 彭钏, 杨腾, 崔梦琪, 孙伟成, 张剑坛, 刘皓天, 巴超群, 王鹤琪, 贾竞超, 武金洲, 肖翠, 马克平. 国内 8 款常用植物识别软件的识别能力评价. 生物多样性, 2020, 28 (4):524-533. http://www.biodiversityscience.net/CN/10.17520/biods.2019272

\begin{tabular}{|c|c|}
\hline 物种 & 照片在 NSII 网页的地址 \\
\hline \multicolumn{2}{|l|}{ 14.狝猴桃科 Actinidiaceae } \\
\hline \multicolumn{2}{|l|}{ 1.猕猴桃属 Actinidia } \\
\hline 1.小叶猕猴桃 Actinidia lanceolata & $\begin{array}{l}\text { http://www.nsii.org.cn/nsiiresources/media/photo/testPhoto/3c/original/Actinidia lanceolata_小叶猕猴桃_浙江古田 } \\
\text { 山_陈涁 2008-05-15 14-26-14.jpg }\end{array}$ \\
\hline \multicolumn{2}{|l|}{ 15.马醜铃科 Aristolochiaceae } \\
\hline \multicolumn{2}{|l|}{ 1.马兒铃属 Aristolochia } \\
\hline 1.管花马兒铃 Aristolochia tubiflora & $\begin{array}{l}\text { http://www.nsii.org.cn/nsiiresources/media/photo/testPhoto/3c/original/Aristolochia tubiflora_管花马兒铃_浙江古田 } \\
\text { 山_陈涁 2008-05-12 17-13-15.jpg }\end{array}$ \\
\hline \multicolumn{2}{|l|}{ 2.细辛属 Asarum } \\
\hline 1.祁阳细辛 Asarum magnificum & $\begin{array}{l}\text { http://www.nsii.org.cn/nsiiresources/media/photo/testPhoto/3c/original/Asarum magnificum_祁阳细辛_浙江古田山_ } \\
\text { 陈涁 2008-05-12 17-33-14.jpg }\end{array}$ \\
\hline \multicolumn{2}{|l|}{ 16.山茶科 Theaceae } \\
\hline \multicolumn{2}{|l|}{ 1.柃木属 Eurya } \\
\hline 1.格药柃 Eurya muricata & $\begin{array}{l}\text { http://www.nsii.org.cn/nsiiresources/media/photo/testPhoto/3c/original/Eurya muricata_格药柃_浙江古田山_陈涁 } \\
\text { 2008-05-14 13-34-30.jpg }\end{array}$ \\
\hline \multicolumn{2}{|l|}{ 2.杨桐属 Adinandra } \\
\hline 1.杨桐 Adinandra millettii & $\begin{array}{l}\text { http://www.nsii.org.cn/nsiiresources/media/photo/testPhoto/3c/original/Adinandra millettii_杨桐_浙江古田山_陈涁 } \\
\text { 2008-05-13 09-33-07.jpg }\end{array}$ \\
\hline \multicolumn{2}{|l|}{ 3.山茶属 Camellia } \\
\hline $\begin{array}{l}\text { 1.浙江红山茶 Camellia } \\
\text { chekiangoleosa }\end{array}$ & $\begin{array}{l}\text { http://www.nsii.org.cn/nsiiresources/media/photo/testPhoto/3c/original/Camellia chekiangoleosa_浙江红山茶_浙江 } \\
\text { 古田山_陈涁 2008-05-14 09-22-32.jpg }\end{array}$ \\
\hline 2. 茶 Camellia sinensis & $\begin{array}{l}\text { http://www.nsii.org.cn/nsiiresources/media/photo/testPhoto/3c/original/Camellia sinensis_茶_浙江古田山_陈彬 } \\
\text { 2008-05-14 16-55-27.jpg }\end{array}$ \\
\hline \multicolumn{2}{|l|}{ 17.十字花科 Cruciferae } \\
\hline \multicolumn{2}{|l|}{ 1.芸苔属 Brassica } \\
\hline 1.欧洲油菜 Brassica napus & $\begin{array}{l}\text { http://www.nsii.org.cn/nsiiresources/media/photo/testPhoto/3c/original/Brassica napus_欧洲油菜_浙江古田山_陈彬 } \\
\text { 2008-05-13 09-55-40.jpg }\end{array}$ \\
\hline \multicolumn{2}{|l|}{ 18.虎耳草科 Saxifragaceae } \\
\hline \multicolumn{2}{|l|}{ 1.绣球属 Hydrangea } \\
\hline 1.中国绣球 Hydrangea chinensis & $\begin{array}{l}\text { http://www.nsii.org.cn/nsiiresources/media/photo/testPhoto/3c/original/Hydrangea chinensis_中国绣球_浙江古田山 } \\
\text { _陈涁 2008-05-12 17-23-20.jpg }\end{array}$ \\
\hline 2.中国绣球 Hydrangea chinensis & $\begin{array}{l}\text { http://www.nsii.org.cn/nsiiresources/media/photo/testPhoto/3c/original/Hydrangea chinensis_中国绣球_浙江古田山 } \\
\text { _陈涁 2008-05-13 16-04-21.jpg }\end{array}$ \\
\hline 3.中国绣球 Hydrangea chinensis & $\begin{array}{l}\text { http://www.nsii.org.cn/nsiiresources/media/photo/testPhoto/3c/original/Hydrangea chinensis_中国绣球_浙江古田山 } \\
\text { _陈涁 2008-05-13 16-04-28.jpg }\end{array}$ \\
\hline 4.中国绣球 Hydrangea chinensis & $\begin{array}{l}\text { http://www.nsii.org.cn/nsiiresources/media/photo/testPhoto/3c/original/Hydrangea chinensis_中国绣球_浙江古田山 } \\
\text { _陈涁 2008-05-13 16-04-38.jpg }\end{array}$ \\
\hline 5.中国绣球 Hydrangea chinensis & $\begin{array}{l}\text { http://www.nsii.org.cn/nsiiresources/media/photo/testPhoto/3c/original/Hydrangea chinensis_中国绣球_浙江古田山 } \\
\text { _陈涁 2008-05-14 09-38-00.jpg }\end{array}$ \\
\hline \multicolumn{2}{|l|}{ 19.菩薇科 Rosaceae } \\
\hline 1.樱属 Cerasus & \\
\hline
\end{tabular}


许展慧, 刘诗尧, 赵芗, 涂文琴, 常诏峰, 张恩涛, 郭靖, 郑迪, 耿釷, 顾高营, 郭淳鹏, 郭璐璐, 王静, 徐春 阳, 彭钏, 杨腾, 崔梦琪, 孙伟成, 张剑坛, 刘皓天, 巴超群, 王鹤琪, 贾竞超, 武金洲, 肖翠, 马克平. 国内 8 款常用植物识别软件的识别能力评价. 生物多样性, 2020, 28 (4):524-533. http://www.biodiversityscience.net/CN/10.17520/biods.2019272

\begin{tabular}{|c|c|}
\hline 物种 & 照片在 NSII 网页的地址 \\
\hline 1.浙闽樱桃 Cerasus schneideriana & $\begin{array}{l}\text { http://www.nsii.org.cn/nsiiresources/media/photo/testPhoto/3c/original/Cerasus schneideriana_浙闽樱桃_浙江古田 } \\
\text { 山_陈涁 2008-05-13 11-44-26.jpg }\end{array}$ \\
\hline \multicolumn{2}{|l|}{ 20.豆科 Fabaceae } \\
\hline \multicolumn{2}{|l|}{ 1.仪花属 Lysidice } \\
\hline 1.仪花 Lysidice rhodostegia & http://www.nsii.org.cn/nsiiresources/media/photo/testPhoto/3c/original/仪花 Lysidice rhodostegia.jpg \\
\hline \multicolumn{2}{|l|}{ 21.槭树科 Aceraceae } \\
\hline \multicolumn{2}{|l|}{ 1.槭属 Acer } \\
\hline 1.阔叶槭 Acer amplum & $\begin{array}{l}\text { http://www.nsii.org.cn/nsiiresources/media/photo/testPhoto/3c/original/Acer amplum_阔叶槭_浙江古田山_陈涁 } \\
\text { 2008-05-14 15-00-26.jpg }\end{array}$ \\
\hline 2.紫果槭 Acer cordatum & $\begin{array}{l}\text { http://www.nsii.org.cn/nsiiresources/media/photo/testPhoto/3c/original/Acer cordatum_紫果棫_浙江古田山_陈涁 } \\
\text { 2008-05-14 17-07-56.jpg }\end{array}$ \\
\hline 3.青榨棫 Acer davidii & $\begin{array}{l}\text { http://www.nsii.org.cn/nsiiresources/media/photo/testPhoto/3c/original/Acer davidii_青榨槭_浙江古田山_陈彬 } \\
\text { 2008-05-14 14-27-39.jpg }\end{array}$ \\
\hline 4.秀丽槭 Acer elegantulum & $\begin{array}{l}\text { http://www.nsii.org.cn/nsiiresources/media/photo/testPhoto/3c/original/Acer elegantulum_秀丽械_浙江古田山_陈涁 } \\
\text { 2008-05-13 08-43-49.jpg }\end{array}$ \\
\hline 5.鸡爪槭 Acer palmatum & $\begin{array}{l}\text { http://www.nsii.org.cn/nsiiresources/media/photo/testPhoto/3c/original/Acer palmatum_鸡爪棫_浙江古田山_陈涁 } \\
\text { 2008-05-16 11-06-17.jpg }\end{array}$ \\
\hline \multicolumn{2}{|l|}{ 22.冬青科 Aquifoliaceae } \\
\hline \multicolumn{2}{|l|}{ 1.冬青属 Ilex } \\
\hline 1.大叶冬青 Ilex latifolia & $\begin{array}{l}\text { http://www.nsii.org.cn/nsiiresources/media/photo/testPhoto/3c/original/Ilex latifolia_大叶冬青_浙江古田山_陈涁 } \\
\text { 2008-05-16 09-29-39.jpg }\end{array}$ \\
\hline 2.大叶冬青 Ilex latifolia & $\begin{array}{l}\text { http://www.nsii.org.cn/nsiiresources/media/photo/testPhoto/3c/original/Ilex latifolia_大叶冬青_浙江古田山_陈涁 } \\
\text { 2008-05-16 09-30-04.jpg }\end{array}$ \\
\hline 2.木姜冬青 Ilex litseifolia & $\begin{array}{l}\text { http://www.nsii.org.cn/nsiiresources/media/photo/testPhoto/3c/original/Ilex litseifolia_木姜冬青_浙江古田山_陈涁 } \\
\text { 2008-05-14 17-10-43.jpg }\end{array}$ \\
\hline \multicolumn{2}{|l|}{ 23.卫矛科 Celastraceae } \\
\hline \multicolumn{2}{|l|}{ 1.南蛇藤属 Celastrus } \\
\hline 1.窄叶南蛇藤 Celastrus oblanceifolius & $\begin{array}{l}\text { http://www.nsii.org.cn/nsiiresources/media/photo/testPhoto/3c/original/Celastrus oblanceifolius_窄叶南蛇藤_浙江古 } \\
\text { 田山_陈涁 2008-05-14 11-19-32.jpg }\end{array}$ \\
\hline \multicolumn{2}{|l|}{ 24.省沽油科 Staphyleaceae } \\
\hline \multicolumn{2}{|l|}{ 1.野鸦椿属 Euscaphis } \\
\hline 1.野鸦椿 Euscaphis japonica & $\begin{array}{l}\text { http://www.nsii.org.cn/nsiiresources/media/photo/testPhoto/3c/original/Euscaphis japonica_野鸦椿_浙江古田山_陈 } \\
\text { 涁 2008-05-13 09-47-53.jpg }\end{array}$ \\
\hline \multicolumn{2}{|l|}{ 25.金栗兰科 Chloranthaceae } \\
\hline \multicolumn{2}{|l|}{ 1.金栗兰属 Chloranthus } \\
\hline 1.及已 Chloranthus serratus & $\begin{array}{l}\text { http://www.nsii.org.cn/nsiiresources/media/photo/testPhoto/3c/original/Chloranthus serratus_及己_浙江古田山_陈涁 } \\
\text { 2008-05-12 17-08-46.jpg }\end{array}$ \\
\hline \multicolumn{2}{|l|}{ 26.大风子科 Flacourtiaceae } \\
\hline \multicolumn{2}{|l|}{ 1.山桐子属 Idesia } \\
\hline 1.山桐子 Idesia polycarpa & $\begin{array}{l}\text { http://www.nsii.org.cn/nsiiresources/media/photo/testPhoto/3c/original/Idesia polycarpa_山桐子_浙江古田山_陈涁 } \\
\text { 2008-05-13 15-01-37.jpg }\end{array}$ \\
\hline
\end{tabular}


许展慧, 刘诗尧, 赵莹, 涂文琴, 常诏峰, 张恩涛, 郭靖, 郑迪, 耿均, 顾高营, 郭淳鹏, 郭璐璐, 王静, 徐春 阳, 彭钏, 杨腾, 崔梦琪, 孙伟成, 张剑坛, 刘皓天, 巴超群, 王鹤琪, 贾竞超, 武金洲, 肖翠, 马克平. 国内 8 款常用植物识别软件的识别能力评价. 生物多样性, 2020, 28 (4):524-533. http://www.biodiversityscience.net/CN/10.17520/biods.2019272

\begin{tabular}{|c|c|}
\hline 物种 & 照片在 NSII 网页的地址 \\
\hline 2.山桐子 Idesia polycarpa & $\begin{array}{l}\text { http://www.nsii.org.cn/nsiiresources/media/photo/testPhoto/3c/original/Idesia polycarpa_山桐子_浙江古田山_陈涁 } \\
\text { 2008-05-13 15-01-40.jpg }\end{array}$ \\
\hline 3.山桐子 Idesia polycarpa & $\begin{array}{l}\text { http://www.nsii.org.cn/nsiiresources/media/photo/testPhoto/3c/original/Idesia polycarpa_山桐子_浙江古田山_陈涁 } \\
\text { 2008-05-13 15-02-12.jpg }\end{array}$ \\
\hline \multicolumn{2}{|l|}{ 27.小二仙草科 Haloragidaceae } \\
\hline \multicolumn{2}{|l|}{ 1.小二仙草属 Haloragis } \\
\hline 1.小二仙草 Haloragis micrantha & $\begin{array}{l}\text { http://www.nsii.org.cn/nsiiresources/media/photo/testPhoto/3c/original/Gonocarpus micranthus_小二仙草_浙江古田 } \\
\text { 山_陈涁 2008-05-14 13-57-38.jpg }\end{array}$ \\
\hline \multicolumn{2}{|l|}{ 28.桤叶树科 Clethraceae } \\
\hline \multicolumn{2}{|l|}{ 1.桤叶树属 Clethra } \\
\hline 1.髡脉桤叶树 Clethra barbinervis & $\begin{array}{l}\text { http://www.nsii.org.cn/nsiiresources/media/photo/testPhoto/3c/original/Clethra barbinervis_髭脉桤叶树_浙江古田山 } \\
\text { _陈涁 2008-05-14 13-14-34.jpg }\end{array}$ \\
\hline \multicolumn{2}{|l|}{ 29.虎皮楠科 Daphniphyllaceae } \\
\hline \multicolumn{2}{|l|}{ 1.虎皮楠属 Daphniphyllum } \\
\hline 1.交让木 Daphniphyllum macropodum & $\begin{array}{l}\text { http://www.nsii.org.cn/nsiiresources/media/photo/testPhoto/3c/original/Daphniphyllum macropodum_交让木_浙江 } \\
\text { 古田山_陈彬 2008-05-14 13-17-45.jpg }\end{array}$ \\
\hline 2.虎皮楠 Daphniphyllum oldhami & $\begin{array}{l}\text { http://www.nsii.org.cn/nsiiresources/media/photo/testPhoto/3c/original/Daphniphyllum oldhami_浙江古田山_陈涁 } \\
\text { 2008-05-15 16-43-46.jpg }\end{array}$ \\
\hline \multicolumn{2}{|l|}{ 30.八角枫科 Alangiaceae } \\
\hline \multicolumn{2}{|l|}{ 1.八角枫属 Alangium } \\
\hline 1.八角枫 Alangium chinense & $\begin{array}{l}\text { http://www.nsii.org.cn/nsiiresources/media/photo/testPhoto/3c/original/Alangium chinense_八角枫_浙江古田山_陈 } \\
\text { 涁 2008-05-13 15-50-46.jpg }\end{array}$ \\
\hline \multicolumn{2}{|l|}{ 31.鼠李科 Rhamnaceae } \\
\hline \multicolumn{2}{|l|}{ 1.勾儿茶属 Berchemia } \\
\hline 1.多花勾儿茶 Berchemia floribunda & $\begin{array}{l}\text { http://www.nsii.org.cn/nsiiresources/media/photo/testPhoto/3c/original/Berchemia floribunda_多花勾儿茶_浙江古田 } \\
\text { 山_陈涁 2008-05-13 08-52-03.jpg }\end{array}$ \\
\hline \multicolumn{2}{|l|}{ 32.葡萄科 Vitaceae } \\
\hline \multicolumn{2}{|l|}{ 1.蛇葡萄属 Ampelopsis } \\
\hline 1.广东蛇葡萄 Ampelopsis cantoniensis & $\begin{array}{l}\text { http://www.nsii.org.cn/nsiiresources/media/photo/testPhoto/3c/original/Ampelopsis cantoniensis_广东蛇葡萄_浙江 } \\
\text { 古田山_陈涁 2008-05-13 08-22-49.jpg }\end{array}$ \\
\hline 2.白蔹 Ampelopsis japonica & $\begin{array}{l}\text { http://www.nsii.org.cn/nsiiresources/media/photo/testPhoto/3c/original/Ampelopsis japonica_白蔹_浙江古田山_陈 } \\
\text { 涁 2008-05-14 07-24-28.jpg }\end{array}$ \\
\hline \multicolumn{2}{|l|}{ 2.乌蔹莓属 Cayratia } \\
\hline 1.角花乌蔹莓 Cayratia corniculata & $\begin{array}{l}\text { http://www.nsii.org.cn/nsiiresources/media/photo/testPhoto/3c/original/Cayratia corniculata_角花乌蔹莓_浙江古田 } \\
\text { 山_陈彬 2008-05-13 16-14-10.jpg }\end{array}$ \\
\hline \multicolumn{2}{|l|}{ 33.杜英科 Elaeocarpaceae } \\
\hline \multicolumn{2}{|l|}{ 1.杜英属 Elaeocarpus } \\
\hline 1.中华杜英 Elaeocarpus chinensis & $\begin{array}{l}\text { http://www.nsii.org.cn/nsiiresources/media/photo/testPhoto/3c/original/Elaeocarpus chinensis_中华杜英_浙江古田 } \\
\text { 山_陈涁 2008-05-13 10-38-25.jpg }\end{array}$ \\
\hline \multicolumn{2}{|l|}{ 34.紫金牛科 Myrsinaceae } \\
\hline 1.紫金牛属 Ardisia & \\
\hline
\end{tabular}


许展慧, 刘诗尧, 赵莹, 涂文琴, 常诏峰, 张恩涛, 郭靖, 郑迪, 耿均, 顾高营, 郭淳鹏, 郭璐璐, 王静, 徐春 阳, 彭钏, 杨腾, 崔梦琪, 孙伟成, 张剑坛, 刘皓天, 巴超群, 王鹤琪, 贾竞超, 武金洲, 肖翠, 马克平. 国内 8 款常用植物识别软件的识别能力评价. 生物多样性, 2020, 28 (4):524-533. http://www.biodiversityscience.net/CN/10.17520/biods.2019272

\begin{tabular}{|c|c|}
\hline 物种 & 照片在 NSII 网页的地址 \\
\hline 1.网脉酸藤子 Embelia rudis & $\begin{array}{l}\text { http://www.nsii.org.cn/nsiiresources/media/photo/testPhoto/3c/original/Embelia rudis_网脉酸藤子_浙江古田山_陈 } \\
\text { 涁 2008-05-13 10-39-38.jpg }\end{array}$ \\
\hline \multicolumn{2}{|l|}{ 35.安息香科 Styracaceae } \\
\hline \multicolumn{2}{|l|}{ 1.赤杨叶属 Alniphyllum } \\
\hline 1.赤杨叶 Alniphyllum fortunei & $\begin{array}{l}\text { http://www.nsii.org.cn/nsiiresources/media/photo/testPhoto/3c/original/Alniphyllum fortunei_赤杨叶_浙江古田山_ } \\
\text { 陈涁 2008-05-14 15-38-43.jpg }\end{array}$ \\
\hline \multicolumn{2}{|l|}{ 36.木樨科 Oleaceae } \\
\hline \multicolumn{2}{|l|}{ 1.梣属 Fraxinus } \\
\hline 1.苦枥木 Fraxinus insularis & $\begin{array}{l}\text { http://www.nsii.org.cn/nsiiresources/media/photo/testPhoto/3c/original/Fraxinus insularis_苦枥木_浙江古田山_陈涁 } \\
\text { 2008-05-14 12-53-01.jpg }\end{array}$ \\
\hline 2.茉莉花 Jasminum sambac & http://www.nsii.org.cn/nsiiresources/media/photo/testPhoto/3c/original/茉莉花 Jasminum sambac.jpg \\
\hline \multicolumn{2}{|l|}{ 2.素馨属 Jasminum } \\
\hline 1.粗糠树 Ehretia macrophylla & $\begin{array}{l}\text { http://www.nsii.org.cn/nsiiresources/media/photo/testPhoto/3c/original/Ehretia macrophylla_粗糠树_浙江古田山_陈 } \\
\text { 涁 2008-05-12 17-16-33.jpg }\end{array}$ \\
\hline \multicolumn{2}{|l|}{ 37.苦苣苔科 Gesneriaceae } \\
\hline \multicolumn{2}{|l|}{ 1.粗筒芦苔属 Briggsia } \\
\hline 1.浙皖粗筒苣苔 Briggsia chienii & $\begin{array}{l}\text { http://www.nsii.org.cn/nsiiresources/media/photo/testPhoto/3c/original/Briggsia chienii_浙皖粗筒苣苔_浙江古田山 } \\
\text { _陈涁 2008-05-13 11-16-17.jpg }\end{array}$ \\
\hline \multicolumn{2}{|l|}{ 2.唇柱苣苔属 Chirita } \\
\hline 1.羽裂唇柱苣苔 Chirita pinnatifida & $\begin{array}{l}\text { http://www.nsii.org.cn/nsiiresources/media/photo/testPhoto/3c/original/Chirita pinnatifida_羽裂唇柱苣苔_浙江古田 } \\
\text { 山_陈涁 2008-05-13 15-38-50.jpg }\end{array}$ \\
\hline \multicolumn{2}{|l|}{ 38.桔梗科 Campanulaceae } \\
\hline \multicolumn{2}{|l|}{ 1.沙参属 Adenophora } \\
\hline 1.荠苨 Adenophora trachelioides & $\begin{array}{l}\text { http://www.nsii.org.cn/nsiiresources/media/photo/testPhoto/3c/original/Adenophora trachelioides_荠苨_浙江古田山 } \\
\text { _陈涁 2008-05-16 10-57-31.jpg }\end{array}$ \\
\hline \multicolumn{2}{|l|}{ 2.党参属 Codonopsis } \\
\hline 1.羊乳 Codonopsis lanceolata & $\begin{array}{l}\text { http://www.nsii.org.cn/nsiiresources/media/photo/testPhoto/3c/original/Codonopsis lanceolata_羊乳_浙江古田山_陈 } \\
\text { 涁 2008-05-13 17-17-56.jpg }\end{array}$ \\
\hline \multicolumn{2}{|l|}{ 39.菊科 Compositae } \\
\hline \multicolumn{2}{|l|}{ 1.下田菊属 Adenostemma } \\
\hline 1.下田菊 Adenostemma lavenia & $\begin{array}{l}\text { http://www.nsii.org.cn/nsiiresources/media/photo/testPhoto/3c/original/Adenostemma lavenia_下田菊_浙江古田山_ } \\
\text { 陈涁 2008-05-12 17-04-21.jpg }\end{array}$ \\
\hline \multicolumn{2}{|l|}{ 2.兔儿风属 Ainsliaea } \\
\hline $\begin{array}{l}\text { 1.阿里山兔儿风 Ainsliaea } \\
\text { macroclinidioides }\end{array}$ & $\begin{array}{l}\text { http://www.nsii.org.cn/nsiiresources/media/photo/testPhoto/3c/original/Ainsliaea macroclinidioides_阿里山兔儿风_ } \\
\text { 浙江古田山_陈涁 2008-05-14 07-30-36.jpg }\end{array}$ \\
\hline \multicolumn{2}{|l|}{ 3.蒿属 Artemisia } \\
\hline 1.南牡蒿 Artemisia eriopoda & $\begin{array}{l}\text { http://www.nsii.org.cn/nsiiresources/media/photo/testPhoto/3c/original/Artemisia eriopoda_南牡蒿_浙江古田山_陈 } \\
\text { 涁 2008-05-15 16-23-32.jpg }\end{array}$ \\
\hline \multicolumn{2}{|l|}{ 4.紫苑属 Aster } \\
\hline 1.三脉紫苑 Aster ageratoides & $\begin{array}{l}\text { http://www.nsii.org.cn/nsiiresources/media/photo/testPhoto/3c/original/Aster ageratoides_三脉紫苑_浙江古田山_陈 } \\
\text { 涁 2008-05-12 17-33-37.jpg }\end{array}$ \\
\hline 5.䔡属 Cirsium & \\
\hline
\end{tabular}


许展慧, 刘诗尧, 赵芗, 涂文琴, 常诏峰, 张恩涛, 郭靖, 郑迪, 耿釷, 顾高营, 郭淳鹏, 郭璐璐, 王静, 徐春 阳, 彭钏, 杨腾, 崔梦琪, 孙伟成, 张剑坛, 刘皓天, 巴超群, 王鹤琪, 贾竞超, 武金洲, 肖翠, 马克平. 国内 8 款常用植物识别软件的识别能力评价. 生物多样性, 2020, 28 (4):524-533. http://www.biodiversityscience.net/CN/10.17520/biods.2019272

\begin{tabular}{|c|c|}
\hline 物种 & 照片在 NSII 网页的地址 \\
\hline 1.蓟 Cirsium japonicum & $\begin{array}{l}\text { http://www.nsii.org.cn/nsiiresources/media/photo/testPhoto/3c/original/Cirsium japonicum_蓟_浙江古田山_陈涁 } \\
\text { 2008-05-12 16-44-19.jpg }\end{array}$ \\
\hline \multicolumn{2}{|l|}{ 6.野茼蒿属 Crassocephalum } \\
\hline 1.野茼蒿 Crassocephalum crepidioides & $\begin{array}{l}\text { http://www.nsii.org.cn/nsiiresources/media/photo/testPhoto/3c/original/Crassocephalum crepidioides_野茼蒿_浙江古 } \\
\text { 田山_陈涁 2008-05-15 17-00-27.jpg }\end{array}$ \\
\hline \multicolumn{2}{|l|}{ 7.黄瓜菜属 Crepidiastrum } \\
\hline 1.黄瓜菜 Crepidiastrum denticulatum & $\begin{array}{l}\text { http://www.nsii.org.cn/nsiiresources/media/photo/testPhoto/3c/original/Crepidiastrum denticulatum_黄瓜菜_浙江古 } \\
\text { 田山_陈涁 2008-05-12 17-00-37.jpg }\end{array}$ \\
\hline \multicolumn{2}{|l|}{ 8.飞蓬属 Erigeron } \\
\hline 1.一年蓬 Erigeron annuus & $\begin{array}{l}\text { http://www.nsii.org.cn/nsiiresources/media/photo/testPhoto/3c/original/Erigeron annuus_一年蓬_浙江古田山_陈彬 } \\
\text { 2008-05-12 17- 13-27.jpg }\end{array}$ \\
\hline 2.一年蓬 Erigeron annuus & $\begin{array}{l}\text { http://www.nsii.org.cn/nsiiresources/media/photo/testPhoto/3c/original/Erigeron annuus_一年蓬_浙江古田山_陈涁 } \\
\text { 2008-05-12 17-29-37.jpg }\end{array}$ \\
\hline \multicolumn{2}{|l|}{ 40.百合科 Liliaceae } \\
\hline \multicolumn{2}{|l|}{ 1.粉条儿菜属 Aletris } \\
\hline 1.粉条儿菜 Aletris spicata & $\begin{array}{l}\text { http://www.nsii.org.cn/nsiiresources/media/photo/testPhoto/3c/original/Aletris spicata_粉条儿菜_浙江古田山_陈彬 } \\
\text { 2008-05-14 09-33-09.jpg }\end{array}$ \\
\hline \multicolumn{2}{|l|}{ 2.萱草属 Hemerocallis } \\
\hline 1.萱草 Hemerocallis fulva & $\begin{array}{l}\text { http://www.nsii.org.cn/nsiiresources/media/photo/testPhoto/3c/original/Hemerocallis fulva_萱草_浙江古田山_陈涁 } \\
\text { 2008-05-14 11-49-03.jpg }\end{array}$ \\
\hline \multicolumn{2}{|l|}{ 41.薯蓣科 Dioscoreaceae } \\
\hline \multicolumn{2}{|l|}{ 1.薯蓣属 Dioscorea } \\
\hline 1.日本薯蓣 Dioscorea japonica & $\begin{array}{l}\text { http://www.nsii.org.cn/nsiiresources/media/photo/testPhoto/3c/original/Dioscorea japonica_日本薯蓣_浙江古田山_ } \\
\text { 陈涁 2008--05-15 15-32-14.jpg }\end{array}$ \\
\hline 2.日本薯蓣 Dioscorea japonica & $\begin{array}{l}\text { http://www.nsii.org.cn/nsiiresources/media/photo/testPhoto/3c/original/Dioscorea japonica_日本薯蓣_浙江古田山_ } \\
\text { 陈涁 2008-05-13 16-32-32.jpg }\end{array}$ \\
\hline \multicolumn{2}{|l|}{ 42.天南星科 Araceae } \\
\hline \multicolumn{2}{|l|}{ 1.菖蒲属 Acorus } \\
\hline 1.金钱蒲 Acorus gramineus & $\begin{array}{l}\text { http://www.nsii.org.cn/nsiiresources/media/photo/testPhoto/3c/original/Acorus gramineus_金钱蒲_浙江古田山_陈 } \\
\text { 涁 2008-05-15 14-32-22.jpg }\end{array}$ \\
\hline \multicolumn{2}{|l|}{ 2.天南星属 Arisaema } \\
\hline 1.灯台莲 Arisaema bockii & $\begin{array}{l}\text { http://www.nsii.org.cn/nsiiresources/media/photo/testPhoto/3c/original/Arisaema bockii_灯台莲_浙江古田山_陈涁 } \\
\text { 2008-05-16 10-09-28.jpg }\end{array}$ \\
\hline 2.一把伞南星 Arisaema erubescens & $\begin{array}{l}\text { http://www.nsii.org.cn/nsiiresources/media/photo/testPhoto/3c/original/Arisaema erubescens_一把伞南星_浙江古田 } \\
\text { 山_陈涁 2008-05-14 11-21-53.jpg }\end{array}$ \\
\hline \multicolumn{2}{|l|}{ 43.姜科 Zingiberaceae } \\
\hline \multicolumn{2}{|l|}{ 1.山姜属 Alpinia } \\
\hline 1.山姜 Alpinia japonica & $\begin{array}{l}\text { http://www.nsii.org.cn/nsiiresources/media/photo/testPhoto/3c/original/Alpinia japonica_山姜_浙江古田山_陈涁 } \\
\text { 2008-05-13 08-32-24.jpg }\end{array}$ \\
\hline 2.山姜 Alpinia japonica & $\begin{array}{l}\text { http://www.nsii.org.cn/nsiiresources/media/photo/testPhoto/3c/original/Alpinia japonica_山姜_浙江古田山_陈涁 } \\
\text { 2008-05-13 08-35-27.jpg }\end{array}$ \\
\hline 44.兰科 Orchidaceae & \\
\hline
\end{tabular}


许展慧, 刘诗尧, 赵莹, 涂文琴, 常诏峰, 张恩涛, 郭靖, 郑迪, 耿均, 顾高营, 郭淳鹏, 郭璐璐, 王静, 徐春 阳, 彭钏, 杨腾, 崔梦琪, 孙伟成, 张剑坛, 刘皓天, 巴超群, 王鹤琪, 贾竞超, 武金洲, 肖翠, 马克平. 国内 8 款常用植物识别软件的识别能力评价. 生物多样性, 2020, 28 (4):524-533. http://www.biodiversityscience.net/CN/10.17520/biods.2019272

\begin{tabular}{|c|c|}
\hline 物种 & 照片在 NSII 网页的地址 \\
\hline \multicolumn{2}{|l|}{ 1.头芯兰属 Cephalanthera } \\
\hline 1.金兰 Cephalanthera falcata & $\begin{array}{l}\text { http://www.nsii.org.cn/nsiiresources/media/photo/testPhoto/3c/original/Cephalanthera falcata_金兰_浙江古田山_陈 } \\
\text { 涁 2008-05-14 09-23-25.jpg }\end{array}$ \\
\hline 2.金兰 Cephalanthera falcata & $\begin{array}{l}\text { http://www.nsii.org.cn/nsiiresources/media/photo/testPhoto/3c/original/Cephalanthera falcata_金兰_浙江古田山_陈 } \\
\text { 涁 2008-05-14 09-23-28.jpg }\end{array}$ \\
\hline \multicolumn{2}{|l|}{ 2.兰属 Cymbidium } \\
\hline 1.多花兰 Cymbidium floribundum & $\begin{array}{l}\text { http://www.nsii.org.cn/nsiiresources/media/photo/testPhoto/3c/original/Cymbidium floribundum_多花兰_浙江古田 } \\
\text { 山_陈涁 2008-05-15 17-52-42.jpg }\end{array}$ \\
\hline \multicolumn{2}{|l|}{ 四、温带区 } \\
\hline \multicolumn{2}{|l|}{ 1.松科 Pinaceae } \\
\hline \multicolumn{2}{|l|}{ 1.冷杉属 Abies } \\
\hline 1.臭冷杉 Abies nephrolepis & http://www.nsii.org.cn/nsiiresources/media/photo/testPhoto/2x/original/臭冷杉 (2).JPG \\
\hline 2.臭冷杉 Abies nephrolepis & http://www.nsii.org.cn/nsiiresources/media/photo/testPhoto/2x/original/臭冷杉 0882722.JPG \\
\hline \multicolumn{2}{|l|}{ 2.云杉属 Picea } \\
\hline $\begin{array}{l}\text { 1.鱼鳞云杉 Picea jezoensis var. } \\
\text { microsperma }\end{array}$ & http://www.nsii.org.cn/nsiiresources/media/photo/testPhoto/2x/original/鱼鳞云杉 2940.JPG \\
\hline $\begin{array}{l}\text { 2.鱼鳞云杉 Picea jezoensis var. } \\
\text { microsperma }\end{array}$ & http://www.nsii.org.cn/nsiiresources/media/photo/testPhoto/2x/original/鱼鳞云杉 1989.JPG \\
\hline \multicolumn{2}{|l|}{ 3.松属 Pinus } \\
\hline $\begin{array}{l}\text { 1.长白松 Pinus sylvestris var. } \\
\text { sylvestriformis }\end{array}$ & http://www.nsii.org.cn/nsiiresources/media/photo/testPhoto/2x/original/长白松 DSC_0094.JPG \\
\hline 2.红松 Pinus koraiensis & http://www.nsii.org.cn/nsiiresources/media/photo/testPhoto/2x/original/红松 DSC_4347.JPG \\
\hline 3.红松 Pinus koraiensis & http://www.nsii.org.cn/nsiiresources/media/photo/testPhoto/2x/original/红松 周海成 0812513.JPG \\
\hline \multicolumn{2}{|l|}{ 4.落叶松属 Larix } \\
\hline 1.落叶松 Larix gmelinii & http://www.nsii.org.cn/nsiiresources/media/photo/testPhoto/2x/original/落叶松 P6140760.JPG \\
\hline 2.落叶松 Larix gmelinii & http://www.nsii.org.cn/nsiiresources/media/photo/testPhoto/2x/original/落叶松 (22).JPG \\
\hline \multicolumn{2}{|l|}{ 2.桦木科 Betulaceae } \\
\hline \multicolumn{2}{|l|}{ 1.桤木属 Alnus } \\
\hline 1.东北桤木 Alnus mandshurica & http://www.nsii.org.cn/nsiiresources/media/photo/testPhoto/2x/original/东北桤木 DSC_6028.JPG \\
\hline \multicolumn{2}{|l|}{ 2.桦木属 Betula } \\
\hline 1.黑桦 Betula dahurica & http://www.nsii.org.cn/nsiiresources/media/photo/testPhoto/2x/original/黑桦 Betula dahurica 0206.JPG \\
\hline 2.岳桦 Betula ermanii var. ermanii & http://www.nsii.org.cn/nsiiresources/media/photo/testPhoto/2x/original/岳桦 周海成 雌果穗 2000m 7959.JPG \\
\hline 3.白桦 Betula platyphylla & $\begin{array}{l}\text { http://www.nsii.org.cn/nsiiresources/media/photo/testPhoto/2x/original/白桦 Betula platyphylla Betula szechuanica } \\
\text { DSC_2730.JPG }\end{array}$ \\
\hline 3.壳斗科 Fagaceae & +2 \\
\hline \multicolumn{2}{|l|}{ 1.栋属 Quercus } \\
\hline 1.蒙古栋 Quercus mongolica & http://www.nsii.org.cn/nsiiresources/media/photo/testPhoto/2x/original/蒙古栋 种子 4548.JPG \\
\hline \multicolumn{2}{|l|}{ 4.榆科 Ulmaceae } \\
\hline \multicolumn{2}{|l|}{ 1.榆属 Ulmus } \\
\hline 1.春榆 Ulmus davidiana var. japonica & http://www.nsii.org.cn/nsiiresources/media/photo/testPhoto/2x/original/春榆.JPG \\
\hline 2.春榆 Ulmus davidiana var. japonica & $\begin{array}{l}\text { http://www.nsii.org.cn/nsiiresources/media/photo/testPhoto/2x/original/春榆 Ulmus davidiana var japonica } \\
\text { DSCN0098.JPG }\end{array}$ \\
\hline
\end{tabular}


许展慧, 刘诗尧, 赵莹, 涂文琴, 常诏峰, 张恩涛, 郭靖, 郑迪, 耿均, 顾高营, 郭淳鹏, 郭璐璐, 王静, 徐春 阳, 彭钏, 杨腾, 崔梦琪, 孙伟成, 张剑坛, 刘皓天, 巴超群, 王鹤琪, 贾竞超, 武金洲, 肖翠, 马克平. 国内 8 款常用植物识别软件的识别能力评价. 生物多样性, 2020, 28 (4):524-533. http://www.biodiversityscience.net/CN/10.17520/biods.2019272

\begin{tabular}{|c|c|}
\hline 物种 & 照片在 NSII 网页的地址 \\
\hline 3.春榆 Ulmus davidiana var. japonica & $\begin{array}{l}\text { http://www.nsii.org.cn/nsiiresources/media/photo/testPhoto/2x/original/春榆 Ulmus davidiana var japonica } \\
\text { DSCN0097.JPG }\end{array}$ \\
\hline 4. 裂叶榆 Ulmus laciniata & http://www.nsii.org.cn/nsiiresources/media/photo/testPhoto/2x/original/裂叶榆 DSC_0178.jpg \\
\hline \multicolumn{2}{|l|}{ 4.石竹科 Caryophyllaceae } \\
\hline \multicolumn{2}{|l|}{ 1.繁缕属 Stellaria } \\
\hline 1.叉歧繁缕 Stellaria dichotoma & http://www.nsii.org.cn/nsiiresources/media/photo/testPhoto/2x/original/叉歧繁缕 DSC_2335.JPG \\
\hline \multicolumn{2}{|l|}{ 5.木兰科 Magnoliaceae } \\
\hline \multicolumn{2}{|l|}{ 1.五味子属 Schisandra } \\
\hline 1.五味子 Schisandra chinensis & http://www.nsii.org.cn/nsiiresources/media/photo/testPhoto/2x/original/五味子.JPG \\
\hline \multicolumn{2}{|l|}{ 6.毛茛科 Ranunculaceae } \\
\hline \multicolumn{2}{|l|}{ 1.侧金盏花属 Adonis } \\
\hline 1.侧金盏花 Adonis amurensis & http://www.nsii.org.cn/nsiiresources/media/photo/testPhoto/2x/original/侧金盏花 (2).JPG \\
\hline 2.辽吉侧金戞 Adonis pseudoamurensis & http://www.nsii.org.cn/nsiiresources/media/photo/testPhoto/2x/original/辽吉侧金戔.JPG \\
\hline \multicolumn{2}{|l|}{ 2.银莲花属 Anemone } \\
\hline 1.钝裂银莲花 Anemone obtusiloba & http://www.nsii.org.cn/nsiiresources/media/photo/testPhoto/2x/original/钝裂银莲花 SC_3209.JPG \\
\hline \multicolumn{2}{|l|}{ 3.耧斗菜属 Aquilegia } \\
\hline 1.长白耧斗菜 Aquilegia flabellata & $\begin{array}{l}\text { http://www.nsii.org.cn/nsiiresources/media/photo/testPhoto/2x/original/长白耧斗菜 Aquilegia flabellata } \\
\text { DSC_6290.JPG }\end{array}$ \\
\hline 2.长白耧斗菜 Aquilegia flabellata & $\begin{array}{l}\text { http://www.nsii.org.cn/nsiiresources/media/photo/testPhoto/2x/original/长白耧斗菜 Aquilegia flabellata } \\
\text { DSC_5997.JPG }\end{array}$ \\
\hline 3.长白耧斗菜 Aquilegia flabellata & $\begin{array}{l}\text { http://www.nsii.org.cn/nsiiresources/media/photo/testPhoto/2x/original/长白耧斗菜 Aquilegia flabellata } \\
\text { DSC_5984.JPG }\end{array}$ \\
\hline \multicolumn{2}{|l|}{ 4.驴蹄草属 Caltha } \\
\hline 1.马户蹄草 Caltha palustris & http://www.nsii.org.cn/nsiiresources/media/photo/testPhoto/2x/original/驴蹄草 (9).JPG \\
\hline \multicolumn{2}{|l|}{ 5.铁线莲属 Clematis } \\
\hline 1.槭叶铁线莲 Clematis acerifolia & http://www.nsii.org.cn/nsiiresources/media/photo/testPhoto/2x/original/槭叶铁线莲.JPG \\
\hline \multicolumn{2}{|l|}{ 7.狝猴桃科 Actinidiaceae } \\
\hline \multicolumn{2}{|l|}{ 1.狝猴桃属 Actinidia } \\
\hline 1.狗苯猕猴桃 Actinidia kolomikta & http://www.nsii.org.cn/nsiiresources/media/photo/testPhoto/2x/original/狗苯猕猴桃 (1).JPG \\
\hline \multicolumn{2}{|l|}{ 8.罂粟科 Papaveraceae } \\
\hline \multicolumn{2}{|l|}{ 1.荷青花属 Hylomecon } \\
\hline 1.荷青花 Hylomecon japonica & http://www.nsii.org.cn/nsiiresources/media/photo/testPhoto/2x/original/荷青花 (2).JPG \\
\hline \multicolumn{2}{|l|}{ 2.罂粟属 Papaver } \\
\hline 1.高山罂粟 Papaver alpinum & http://www.nsii.org.cn/nsiiresources/media/photo/testPhoto/2x/original/高山罂粟 Papaver alpinum DSC_7247.JPG \\
\hline 2.高山罂粟 Papaver alpinum & http://www.nsii.org.cn/nsiiresources/media/photo/testPhoto/2x/original/高山罂粟 Papaver alpinum DSC_7248.JPG \\
\hline \multicolumn{2}{|l|}{ 9.十字花科 Cruciferae } \\
\hline \multicolumn{2}{|l|}{ 1.念珠芥属 Torularia } \\
\hline 1.蚓果芥 Torularia humilis & http://www.nsii.org.cn/nsiiresources/media/photo/testPhoto/2x/original/蚓果芥.JPG \\
\hline \multicolumn{2}{|l|}{ 10.景天科 Crassulaceae } \\
\hline \multicolumn{2}{|l|}{ 1.红景天属 Rhodiola } \\
\hline $\begin{array}{l}\text { 1.高山红景天 Rhodiola cretinii subsp. } \\
\text { sino-alpina }\end{array}$ & http://www.nsii.org.cn/nsiiresources/media/photo/testPhoto/2x/original/高山红景天 DSC_6100.JPG \\
\hline
\end{tabular}


许展慧, 刘诗尧, 赵芗, 涂文琴, 常诏峰, 张恩涛, 郭靖, 郑迪, 耿釷, 顾高营, 郭淳鹏, 郭璐璐, 王静, 徐春 阳, 彭钏, 杨腾, 崔梦琪, 孙伟成, 张剑坛, 刘皓天, 巴超群, 王鹤琪, 贾竞超, 武金洲, 肖翠, 马克平. 国内 8 款常用植物识别软件的识别能力评价. 生物多样性, 2020, 28 (4):524-533. http://www.biodiversityscience.net/CN/10.17520/biods.2019272

\begin{tabular}{|c|c|}
\hline 物种 & 照片在 NSII 网页的地址 \\
\hline $\begin{array}{l}\text { 2.高山红景天 Rhodiola cretinii subsp. } \\
\text { sino-alpina }\end{array}$ & http://www.nsii.org.cn/nsiiresources/media/photo/testPhoto/2x/original/高山红景天 DSC_6106.JPG \\
\hline $\begin{array}{l}\text { 3.高山红景天 Rhodiola cretinii subsp. } \\
\text { sino-alpina }\end{array}$ & http://www.nsii.org.cn/nsiiresources/media/photo/testPhoto/2x/original/高山红景天 DSC_6477.JPG \\
\hline $\begin{array}{l}\text { 4.红景天 Rhodiola cretinii subsp. sino- } \\
\text { alpina }\end{array}$ & http://www.nsii.org.cn/nsiiresources/media/photo/testPhoto/2x/original/红景天 DSC_2842.JPG \\
\hline \multicolumn{2}{|l|}{ 2.景天属 Sedum } \\
\hline 1.费菜 Sedum aizoon & http://www.nsii.org.cn/nsiiresources/media/photo/testPhoto/2x/original/费菜 DSC_2533.JPG \\
\hline \multicolumn{2}{|l|}{ 11.虎耳草科 Saxifragaceae } \\
\hline \multicolumn{2}{|l|}{ 1.金腰属 Chrysosplenium } \\
\hline $\begin{array}{l}\text { 1.五台金腰 Chrysosplenium } \\
\text { serreanum }\end{array}$ & http://www.nsii.org.cn/nsiiresources/media/photo/testPhoto/2x/original/五台金腰 DSC_3325.JPG \\
\hline \multicolumn{2}{|l|}{ 2.茶蔍子属 Ribes } \\
\hline 1.刺果茶蔍子 Ribes burejense & http://www.nsii.org.cn/nsiiresources/media/photo/testPhoto/2x/original/刺果茶蔍子 DSC_2365.JPG \\
\hline \multicolumn{2}{|l|}{ 12.蓄薇科 Rosaceae } \\
\hline \multicolumn{2}{|l|}{ 1.仙女木属 Dryas } \\
\hline 1.仙女木 Dryas octopetala & http://www.nsii.org.cn/nsiiresources/media/photo/testPhoto/2x/original/仙女木 Dryas octopetala P7011199.JPG \\
\hline 2.仙女木 Dryas octopetala & http://www.nsii.org.cn/nsiiresources/media/photo/testPhoto/2x/original/仙女木 DSC_5843.JPG \\
\hline 3.仙女木 Dryas octopetala & http://www.nsii.org.cn/nsiiresources/media/photo/testPhoto/2x/original/仙女木.JPG \\
\hline \multicolumn{2}{|l|}{ 2.草莓属 Fragaria } \\
\hline 1.野草莓 Fragaria vesca & http://www.nsii.org.cn/nsiiresources/media/photo/testPhoto/2x/original/野草莓.JPG \\
\hline \multicolumn{2}{|l|}{ 3.委陵菜属 Potentilla } \\
\hline 1.银露梅 Potentilla glabra & http://www.nsii.org.cn/nsiiresources/media/photo/testPhoto/2x/original/银露梅 DSC_5663.JPG \\
\hline \multicolumn{2}{|l|}{ 4.蔷薇属 Rosa } \\
\hline 1.刺菩薇 Rosa acicularis & http://www.nsii.org.cn/nsiiresources/media/photo/testPhoto/2x/original/刺蓄薇 DSC_6790.JPG \\
\hline 2.刺蔷薇 Rosa acicularis & http://www.nsii.org.cn/nsiiresources/media/photo/testPhoto/2x/original/刺菩薇 Rosa acicularis P6140724.JPG \\
\hline 3.美菩薇 Rosa bella & http://www.nsii.org.cn/nsiiresources/media/photo/testPhoto/2x/original/美菩薇 DSC_2406.JPG \\
\hline \multicolumn{2}{|l|}{ 5.花揪属 Sorbus } \\
\hline 1.花楸树 Sorbus pohuashanensis & http://www.nsii.org.cn/nsiiresources/media/photo/testPhoto/2x/original/花楸树 DSC_0162.JPG \\
\hline \multicolumn{2}{|l|}{ 6.悬钩子属 Rubus } \\
\hline 1.山楂叶悬钩子 Rubus crataegifolius & http://www.nsii.org.cn/nsiiresources/media/photo/testPhoto/2x/original/山楂叶悬钩子.JPG \\
\hline \multicolumn{2}{|l|}{ 13.豆科 Fabaceae } \\
\hline \multicolumn{2}{|l|}{ 1.马鞍树属 Maackia } \\
\hline 1.朝鲜槐 Maackia amurensis & http://www.nsii.org.cn/nsiiresources/media/photo/testPhoto/2x/original/朝鲜槐 DSC_6656.JPG \\
\hline \multicolumn{2}{|l|}{ 14.槭树科 Aceraceae } \\
\hline \multicolumn{2}{|l|}{ 1.槭属 Acer } \\
\hline 1.族毛槭 Acer barbinerve & http://www.nsii.org.cn/nsiiresources/media/photo/testPhoto/2x/original/簇毛槭 (3).JPG \\
\hline 2.色木槭 Acer mono & http://www.nsii.org.cn/nsiiresources/media/photo/testPhoto/2x/original/色木槭 DSC_0604.JPG \\
\hline 3.假色槭 Acer pseudosieboldianum & http://www.nsii.org.cn/nsiiresources/media/photo/testPhoto/2x/original/假色槭.JPG \\
\hline 4.假色槭 Acer pseudosieboldianum & http://www.nsii.org.cn/nsiiresources/media/photo/testPhoto/2x/original/紫花棫 DSC_3855.JPG \\
\hline 5.花楷棫 Acer ukurunduense & http://www.nsii.org.cn/nsiiresources/media/photo/testPhoto/2x/original/花楷槭 (2).JPG \\
\hline 15.卫矛科 Celastraceae & \\
\hline
\end{tabular}


许展慧, 刘诗尧, 赵莹, 涂文琴, 常诏峰, 张恩涛, 郭靖, 郑迪, 耿均, 顾高营, 郭淳鹏, 郭璐璐, 王静, 徐春 阳, 彭钏, 杨腾, 崔梦琪, 孙伟成, 张剑坛, 刘皓天, 巴超群, 王鹤琪, 贾竞超, 武金洲, 肖翠, 马克平. 国内 8 款常用植物识别软件的识别能力评价. 生物多样性, 2020, 28 (4):524-533. http://www.biodiversityscience.net/CN/10.17520/biods.2019272

\begin{tabular}{|c|c|}
\hline 物种 & 照片在 NSII 网页的地址 \\
\hline \multicolumn{2}{|l|}{ 1.卫矛属 Euonymus } \\
\hline 1.瘤枝卫矛 Euonymus verrucosus & http://www.nsii.org.cn/nsiiresources/media/photo/testPhoto/2x/original/瘤枝卫矛 (11).JPG \\
\hline \multicolumn{2}{|l|}{ 16.五加科 Araliaceae } \\
\hline \multicolumn{2}{|l|}{ 1.五加属 Acanthopanax } \\
\hline 1.刺五加 Acanthopanax senticosus & http://www.nsii.org.cn/nsiiresources/media/photo/testPhoto/2x/original/刺五加 (16).JPG \\
\hline \multicolumn{2}{|l|}{ 17.椴树科 Tiliaceae } \\
\hline \multicolumn{2}{|l|}{ 1.椴树属 Tilia } \\
\hline 1.紫椴 Tilia amurensis & http://www.nsii.org.cn/nsiiresources/media/photo/testPhoto/2x/original/紫椴 DSC_8542.JPG \\
\hline \multicolumn{2}{|l|}{ 18.鹿蹄草科 Pyrolaceae } \\
\hline \multicolumn{2}{|l|}{ 1.鹿蹄草属 Pyrola } \\
\hline 1.红花鹿蹄草 Pyrola incarnata & http://www.nsii.org.cn/nsiiresources/media/photo/testPhoto/2x/original/红花鹿蹄草.jpg \\
\hline 2.红花鹿蹄草 Pyrola incarnata & http://www.nsii.org.cn/nsiiresources/media/photo/testPhoto/2x/original/红花鹿蹄草 DSC_2602.jpg \\
\hline \multicolumn{2}{|l|}{ 2.假水晶兰属 Cheilotheca } \\
\hline 1.球果假水晶兰 Cheilotheca humilis & http://www.nsii.org.cn/nsiiresources/media/photo/testPhoto/2x/original/球果假水晶兰.JPG \\
\hline \multicolumn{2}{|l|}{ 19.杜鹃花科 Ericaceae } \\
\hline \multicolumn{2}{|l|}{ 1.杜香属 Ledum } \\
\hline 1.杜香 Ledum palustre & http://www.nsii.org.cn/nsiiresources/media/photo/testPhoto/2x/original/杜香 DSC_5233.JPG \\
\hline \multicolumn{2}{|l|}{ 2.松毛翠属 Phyllodoce } \\
\hline 1.松毛翠 Phyllodoce caerulea & http://www.nsii.org.cn/nsiiresources/media/photo/testPhoto/2x/original/松毛翠.JPG \\
\hline 2.松毛翠 Phyllodoce caerulea & http://www.nsii.org.cn/nsiiresources/media/photo/testPhoto/2x/original/松毛翠 周海成 9853.JPG \\
\hline \multicolumn{2}{|l|}{ 2.杜鹃属 Rhododendron } \\
\hline 1.牛皮杜鹃 Rhododendron aureum & http://www.nsii.org.cn/nsiiresources/media/photo/testPhoto/2x/original/牛皮杜鹃 周海成 花 9840.JPG \\
\hline 2.牛皮杜鹃 Rhododendron aureum & http://www.nsii.org.cn/nsiiresources/media/photo/testPhoto/2x/original/牛皮杜鹃 周海成 苔原 281.JPG \\
\hline 3.苞叶杜鹃 Rhododendron bracteatum & http://www.nsii.org.cn/nsiiresources/media/photo/testPhoto/2x/original/苞叶杜鹃.JPG \\
\hline 4.苞叶杜鹃 Rhododendron bracteatum & http://www.nsii.org.cn/nsiiresources/media/photo/testPhoto/2x/original/苞叶杜鹃 周海成 花 西大坡 243.JPG \\
\hline 5.照山白 Rhododendron micranthum & http://www.nsii.org.cn/nsiiresources/media/photo/testPhoto/2x/original/照山白ＤSC_2418.JPG \\
\hline \multicolumn{2}{|l|}{ 3.越桔属 Vaccinium } \\
\hline 1.笃斯越桔 Vaccinium uliginosum & http://www.nsii.org.cn/nsiiresources/media/photo/testPhoto/2x/original/笃斯越桔.JPG \\
\hline 2.笃斯越桔 Vaccinium uliginosum & http://www.nsii.org.cn/nsiiresources/media/photo/testPhoto/2x/original/笃斯越橘 DSC_5937.JPG \\
\hline 3.笃斯越枯 Vaccinium uliginosum & http://www.nsii.org.cn/nsiiresources/media/photo/testPhoto/2x/original/笃斯越橘 DSC_5796.JPG \\
\hline 4.笃斯越桔 Vaccinium uliginosum & http://www.nsii.org.cn/nsiiresources/media/photo/testPhoto/2x/original/笃斯越橘 DSC_6813.JPG \\
\hline \multicolumn{2}{|l|}{ 20.报春花科 Primulaceae } \\
\hline \multicolumn{2}{|l|}{ 1.假报春属 Cortusa } \\
\hline 1.假报春 Cortusa matthioli & http://www.nsii.org.cn/nsiiresources/media/photo/testPhoto/2x/original/假报春 DSC_2655.jpg \\
\hline \multicolumn{2}{|l|}{ 2.报春花属 Primula } \\
\hline 1.胭脂花 Primula maximowiczii & http://www.nsii.org.cn/nsiiresources/media/photo/testPhoto/2x/original/胭脂花 DSC_2680.jpg \\
\hline \multicolumn{2}{|l|}{ 20.龙胆科 Gentianaceae } \\
\hline \multicolumn{2}{|l|}{ 1.龙胆属 Gentiana } \\
\hline 1.长白山龙胆 Gentiana jamesii & http://www.nsii.org.cn/nsiiresources/media/photo/testPhoto/2x/original/长白山龙胆.JPG \\
\hline 2.睡菜属 Menyanthes & \\
\hline 1.睡菜 Menyanthes trifoliata & http://www.nsii.org.cn/nsiiresources/media/photo/testPhoto/2x/original/睡菜.JPG \\
\hline 21.木樨科 Oleaceae & \\
\hline
\end{tabular}


许展慧, 刘诗尧, 赵芗, 涂文琴, 常诏峰, 张恩涛, 郭靖, 郑迪, 耿釷, 顾高营, 郭淳鹏, 郭璐璐, 王静, 徐春 阳, 彭钏, 杨腾, 崔梦琪, 孙伟成, 张剑坛, 刘皓天, 巴超群, 王鹤琪, 贾竞超, 武金洲, 肖翠, 马克平. 国内 8 款常用植物识别软件的识别能力评价. 生物多样性, 2020, 28 (4):524-533. http://www.biodiversityscience.net/CN/10.17520/biods.2019272

\begin{tabular}{|c|c|}
\hline 物种 & 照片在 NSII 网页的地址 \\
\hline \multicolumn{2}{|l|}{ 1.梣属 Fraxinus } \\
\hline 1.水曲柳 Fraxinus mandschurica & http://www.nsii.org.cn/nsiiresources/media/photo/testPhoto/2x/original/水曲柳 DSC_6627.JPG \\
\hline \multicolumn{2}{|l|}{ 22.萝藦科 Asclepiadaceae } \\
\hline \multicolumn{2}{|l|}{ 1.我鸟线藤属 Cynanchum } \\
\hline 1.竹灵消 Cynanchum inamoenum & http://www.nsii.org.cn/nsiiresources/media/photo/testPhoto/2x/original/竹灵消 DSC_2449.JPG \\
\hline \multicolumn{2}{|l|}{ 23.花荵科 Polemoniaceae } \\
\hline \multicolumn{2}{|l|}{ 1.花荵属 Polemonium } \\
\hline 1.花荵 Polemonium coeruleum & http://www.nsii.org.cn/nsiiresources/media/photo/testPhoto/2x/original/花荵.JPG \\
\hline \multicolumn{2}{|l|}{ 24.紫草科 Boraginaceae } \\
\hline \multicolumn{2}{|l|}{ 1.勿忘草属 Myosotis } \\
\hline 1.勿忘草 Myosotis silvatica & http://www.nsii.org.cn/nsiiresources/media/photo/testPhoto/2x/original/勿忘草 DSC_2795.jpg \\
\hline \multicolumn{2}{|l|}{ 25.玄参科 Scrophulariaceae } \\
\hline \multicolumn{2}{|l|}{ 1.婆婆纳属 Veronica } \\
\hline $\begin{array}{l}\text { 1.长白婆婆纳 Veronica stelleri var. } \\
\text { longistyla }\end{array}$ & http://www.nsii.org.cn/nsiiresources/media/photo/testPhoto/2x/original/长白婆婆纳.JPG \\
\hline \multicolumn{2}{|l|}{ 26.列当科 Orobanchaceae } \\
\hline \multicolumn{2}{|l|}{ 1.黄筒花属 Phacellanthus } \\
\hline 1.黄筒花 Phacellanthus tubiflorus & http://www.nsii.org.cn/nsiiresources/media/photo/testPhoto/2x/original/黄筒花.JPG \\
\hline \multicolumn{2}{|l|}{ 27.菊科 Compositae } \\
\hline \multicolumn{2}{|l|}{ 1.菊属 Dendranthema } \\
\hline 1.小山菊 Dendranthema oreastrum & http://www.nsii.org.cn/nsiiresources/media/photo/testPhoto/2x/original/小山菊 DSC_6195.JPG \\
\hline 2.小山菊 Dendranthema oreastrum & http://www.nsii.org.cn/nsiiresources/media/photo/testPhoto/2x/original/小山菊.JPG \\
\hline \multicolumn{2}{|l|}{ 2.㯻吾属 Ligularia } \\
\hline 1.狭苞㯻吾 Ligularia intermedia & http://www.nsii.org.cn/nsiiresources/media/photo/testPhoto/2x/original/狭苞㯻吾 DSC_2357.JPG \\
\hline 2.西伯利亚㯻吾 Ligularia sibirica & http://www.nsii.org.cn/nsiiresources/media/photo/testPhoto/2x/original/西伯利亚㯱吾 DSC_3779.jpg \\
\hline \multicolumn{2}{|l|}{ 3.蜂斗菜属 Petasites } \\
\hline 1.蜂斗菜 Petasites japonicus & http://www.nsii.org.cn/nsiiresources/media/photo/testPhoto/2x/original/蜂斗菜.JPG \\
\hline \multicolumn{2}{|l|}{ 4.风毛菊属 Saussurea } \\
\hline 1.高岭风毛菊 Saussurea tomentosa & $\begin{array}{l}\text { http://www.nsii.org.cn/nsiiresources/media/photo/testPhoto/2x/original/高岭风毛菊 Saussurea tomentosa } \\
\text { DSC_5607.JPG }\end{array}$ \\
\hline 2. 高岭风毛菊 Saussurea tomentosa & $\begin{array}{l}\text { http://www.nsii.org.cn/nsiiresources/media/photo/testPhoto/2x/original/高岭风毛菊 Saussurea tomentosa } \\
\text { DSC_6509.JPG }\end{array}$ \\
\hline \multicolumn{2}{|l|}{ 5.款冬属 Tussilago } \\
\hline 1.款冬 Tussilago farfara & http://www.nsii.org.cn/nsiiresources/media/photo/testPhoto/2x/original/款冬.JPG \\
\hline \multicolumn{2}{|l|}{ 28.忍冬科 Caprifoliaceae } \\
\hline \multicolumn{2}{|l|}{ 1.忍冬属 Lonicera } \\
\hline 1.长白忍冬 Lonicera ruprechtiana & http://www.nsii.org.cn/nsiiresources/media/photo/testPhoto/2x/original/长白忍冬 周海成 果 0812111.JPG \\
\hline \multicolumn{2}{|l|}{ 29.百合科 Liliaceae } \\
\hline \multicolumn{2}{|l|}{ 1.铃兰属 Convallaria } \\
\hline 1.铃兰 Convallaria majalis & http://www.nsii.org.cn/nsiiresources/media/photo/testPhoto/2x/original/铃兰.JPG \\
\hline 2. 贝母属 Fritillaria & \\
\hline 1.轮叶贝母 Fritillaria maximowiczii & http://www.nsii.org.cn/nsiiresources/media/photo/testPhoto/2x/original/轮叶贝母.JPG \\
\hline
\end{tabular}


许展慧, 刘诗尧, 赵莹, 涂文琴, 常诏峰, 张恩涛, 郭靖, 郑迪, 耿釷, 顾高营, 郭淳鹏, 郭璐璐, 王静, 徐春 阳, 彭钏, 杨腾, 崔梦琪, 孙伟成, 张剑坛, 刘皓天, 巴超群, 王鹤琪, 贾竞超, 武金洲, 肖翠, 马克平. 国内 8 款常用植物识别软件的识别能力评价. 生物多样性, 2020, 28 (4):524-533. http://www.biodiversityscience.net/CN/10.17520/biods.2019272

\begin{tabular}{|c|c|}
\hline 物种 & 照片在 NSII 网页的地址 \\
\hline \multicolumn{2}{|l|}{ 3.百合属 Lilium } \\
\hline $\begin{array}{l}\text { 1.有斑百合 Lilium concolor var. } \\
\text { pulchellum }\end{array}$ & http://www.nsii.org.cn/nsiiresources/media/photo/testPhoto/2x/original/有斑百合ＤSC_2457.jpg \\
\hline \multicolumn{2}{|l|}{ 4.延龄草属 Trillium } \\
\hline 1.白花延龄草 Trillium kamtschaticum & http://www.nsii.org.cn/nsiiresources/media/photo/testPhoto/2x/original/白花延龄草.JPG \\
\hline \multicolumn{2}{|l|}{ 5.藜芦属 Veratrum } \\
\hline 1.藜芦 Veratrum nigrum & http://www.nsii.org.cn/nsiiresources/media/photo/testPhoto/2x/original/藜芦.JPG \\
\hline \multicolumn{2}{|l|}{ 30.天南星科 Araceae } \\
\hline \multicolumn{2}{|l|}{ 1.犁头尖属 Typhonium } \\
\hline 1.独角莲 Typhonium giganteum & http://www.nsii.org.cn/nsiiresources/media/photo/testPhoto/2x/original/独角莲.jpg \\
\hline \multicolumn{2}{|l|}{ 31.兰科 Orchidaceae } \\
\hline \multicolumn{2}{|l|}{ 1.杓兰属 Cypripedium } \\
\hline 1.大花杓兰 Cypripedium macranthum & http://www.nsii.org.cn/nsiiresources/media/photo/testPhoto/2x/original/大花杓兰.JPG \\
\hline
\end{tabular}

